\title{
Combinatorial methods in cooperative game theory
}

Citation for published version (APA):

Kuipers, J. (1994). Combinatorial methods in cooperative game theory. [Doctoral Thesis, Maastricht University]. Datawyse / Universitaire Pers Maastricht. https://doi.org/10.26481/dis.19940615jk

Document status and date:

Published: 01/01/1994

DOI:

10.26481/dis.19940615jk

Document Version:

Publisher's PDF, also known as Version of record

\section{Please check the document version of this publication:}

- A submitted manuscript is the version of the article upon submission and before peer-review. There can be important differences between the submitted version and the official published version of record.

People interested in the research are advised to contact the author for the final version of the publication, or visit the DOI to the publisher's website.

- The final author version and the galley proof are versions of the publication after peer review.

- The final published version features the final layout of the paper including the volume, issue and page numbers.

Link to publication

\footnotetext{
General rights rights.

- You may freely distribute the URL identifying the publication in the public portal. please follow below link for the End User Agreement:

www.umlib.nl/taverne-license

Take down policy

If you believe that this document breaches copyright please contact us at:

repository@maastrichtuniversity.nl

providing details and we will investigate your claim.
}

Copyright and moral rights for the publications made accessible in the public portal are retained by the authors and/or other copyright owners and it is a condition of accessing publications that users recognise and abide by the legal requirements associated with these

- Users may download and print one copy of any publication from the public portal for the purpose of private study or research.

- You may not further distribute the material or use it for any profit-making activity or commercial gain

If the publication is distributed under the terms of Article $25 \mathrm{fa}$ of the Dutch Copyright Act, indicated by the "Taverne" license above, 


\section{Combinatorial Methods}

in

Cooperative Game Theory 


\section{Combinatorial Methods \\ in \\ Cooperative Game Theory}

\section{PROEFSCHRIFT}

ter verkrijging van de graad van doctor aan de Rijksuniversiteit Limburg te Maastricht, op gezag van de Rector Magnificus, Prof.Dr. H. Philipsen, volgens het besluit van het College van Dekanen, in het openbaar te verdedigen op woensdag 15 juni 1994 om 14.00 uur

door

Jeroen Kuipers

geboren te West Souburg op 7 januari 1963 


\section{Promotores:}

Prof.dr. S.H. Tijs (KU Brabant)

Prof.dr.ir.drs. O.J. Vrieze

\section{Co-promotor:}

Dr. J.J.M. Derks

\section{Beoordelingscommissie:}

Prof.dr.ir. A.W.J. Kolen (voorzitter)

Dr. Y. Crama

Dr. C.G.A.M. van den Nouweland (KU Brabant)

Prol.dr. H.J.M. Peters

Dr. J.A.M. Potters (KU Nijmegen) 


\section{Acknowledgements}

No book is really written by one person. The knowledge and generosity of many must go into it. I wish to thank all persons who helped me one way or another to complete this thesis. Most of all I want to thank Jean Derks. Many results in this thesis are joint work with him. His comments and the discussions with him were essential for the realization of my thesis. It was a pleasure to work with him.

I am grateful to Koos Vrieze for the freedom he gave me to do research according to my own insights, and for involving me in other interesting research-projects. It was a pleasure to work for him and to work with the people in his department.

I want to express my gratitude to Harry Aarts, who interested me in the subject of chapter 6 , and whose knowledge about minimum cost spanning tree games was of great help for writing chapter 4 of this thesis.

Further, I thank Jaques Lenting, Maarten van der Meulen, his wife Inge, and Hans van der Stel for the pleasant dinners and card playing evenings we spent together. I also thank Rossina Slavova who brought me many exciting days.

Finally, I thank my mother for the mental support she gave ine. 



\section{Contents}

1 Games and solution concepts 1

2 Games with restricted cooperation 7

2.1 Restricted cores of games . . . . . . . . . . . 7

2.2 Partitioning games .................... 8

2.3 Perfect graphs and perfect collections . . . . . . . . . . 12

2.4 The total dependency property ............... 15

2.5 The B-prenucleolus . . . . . . . . . . . . . . . 21

2.6 The TD-property and the B-prenucleolus ........ 27

2.7 Location games . . . . . . . . . . . . . . . . . . . . . . . 33

3 Traveling salesman games $\quad 39$

3.1 Introduction . . . . . . . . . . . . . . . . . . . . . . 39

3.2 Routing games . . . . . . . . . . . . . . . . . 41

3.2 .1 Introduction . . . . . . . . . . . . 41

3.2 .2 The anti-core of a routing game . . . . . . 43

3.2.3 Routing games with a non-empty anti-core . . . . . 46

3.3 The 5 -person TSG . . . . . . . . . . . . . . . 50

4 Minimum cost spanning tree games $\quad 59$

4.1 Introduction . . . . . . . . . . . . . . . . . . . . . 59

4.2 The anti-core of MCST games . . . . . . . . . . . . 60

4.3 Information graph games . . . . . . . . . . . . 72

4.3.1 Introduction . . . . . . . . . . . . . . . 72

4.3.2 Concave information graph games . . . . . . . . 73

4.3.3 A description of the anti-core by means of linear constraints . . . . . . . . . . . . 80

4.3.4 The extreme elements of the anti-core . . . . . . 83

4.3.5 The anti-nucleolus of information graph games . . . . 86 
viii

4.4 Minimum cost forest games . . . . . . . . . . . . 92

5 Assignment games 105

5.1 Assignment problems ... . . . . . . . . . . . . 105

5.2 Assignment games . . . . . . . . . . . . . . 111

6 Extreme elements of the core $\quad 115$

6.1 Introduction . . . . . . . . . . . . . . . 115

6.2 Graph induced polytopes ... . . . . . . . . . 121

6.3 Computation of the permutation number . . . . . . 132

Appendix: Graph theoretic notions $\quad 135$

$\begin{array}{lr}\text { Bibliography } & 137\end{array}$

$\begin{array}{lr}\text { Subject index } & 143\end{array}$

$\begin{array}{ll}\text { Author index } & 147\end{array}$

$\begin{array}{lr}\text { Samenvatting } & 149\end{array}$

$\begin{array}{lc}\text { Curriculum Vitae } & 151\end{array}$ 


\section{Chapter 1}

\section{Games and solution concepts}

Let us consider a group of individuals who are all interested in making profit on some market. These individuals can choose to operate on their own, but when two or more individuals coordinate their actions, they will usually make a total profit which is larger than the sum of the individual profits. Clearly, this gives an incentive for people to join in coalitions, where such coordination of actions is possible. However, the formation of a coalition will only take place if its members can come to an agreement on the distribution of the total profit among them. This so called allocation problem can sometimes really obstruct cooperation between people, as is illustrated in the following simple example.

Three people, let us name them Alfred, Bert and Chris, can achieve no profit if they operate on their own. However, if two of them work together, they can obtain 50 dollars together. The situation is even a little bit better if all three of them join in a coalition, because then they can obtain 60 dollars together. It is not easy to predict which coalition will form and how the members of this coalition will distribute the money. Perhaps Alfred and Bert know each other very well. They could propose to Chris that they are willing to cooperate with him, under the condition that Chris gets only 10 dollars of the share. Then Alfred and Bert can equally share the remaining 50 dollars, the same amount they could get without the cooperation of Chris. Maybe Chris simply accepts the proposal. That would be the end of the discussion: Alfred and Bert share the 50 dollars and Chris gets the remaining 10 dollars. Maybe Chris considers the proposal unfair and refuses cooperation. That would also be the end of the discussion, except in this case Chris leaves the negotiations without any money. Another possibility is that Chris tries to test the apparent friendship between. Alfred and Bert by presenting a new 
proposal. In the current proposal Alfred gets 25 dollars and Chris gets 10 dollars. Chris could offer 30 dollars to Alfred if he is willing to join him in a two-person coalition. That would leave 20 dollars for Chris, and both Alfred and Chris would profit in this new proposal. When Alfred accepts the new proposal endless discussions could follow.

Game theory is the branch of mathematics in which situations like the one above are modeled and studied. Several game models exist, some focus on situations where the interests of players are purely conflicting, others study situations where cooperation can benefit the players. In the earlier given example we clearly have a situation of the latter type. A game model which is suited for describing situations like these is the so-called transferable utility game. The term transferable refers to the assumption that players attach the same value to a unit of utility (profit) in the game. Hence, if some utility is transfered from one player to another, the pain of the first player is equal to the gain of the second player, so to speak.

Formally, a tranferable utility garne is a pair $(N, v)$, where $N$ is a nonempty finite set, and $v$ is a real valued function on the power set of $N$, assigning 0 to the empty set. $N$ is called the player set. Its interpretation is that of a set of individuals or players, who are free to cooperate and cluster into coalitions. For any $S \subseteq N$ the worth $v(S)$ is interpreted as the maximal profit or minimal cost that the players in $S$ can achieve if they decide to form a coalition. The function $v$ is called the characteristic function. Throughout this manuscript we speak of a cooperative game or simply a game when we refer to a transferable utility game.

Often it is assumed that all the players who are participating in a game will work together and form the coalition $N$. This leaves us with the problem of finding reasonable rules for distributing the total profit $v(N)$ among the players.

A vector $x=\left(x_{i}\right)_{i \in N} \in \mathbb{R}^{N}$ is called an allocation. The amount $x_{i}$ can be interpreted as the share of the profit which is given to player $i$. In a game $v$ an allocation $x$ is called efficient if it distributes exactly the profit of the coalition $N$ among the players, i.e. if

$$
\sum_{i \in N} x_{i}=v(N)
$$

A widely accepted criterium for an allocation $x$ is that every player should receive at least the amount he can obtain by operating on his own, i.e.

$$
x_{i} \geq v(\{i\}) \text { for all } i \in N \text {. }
$$


Allocations satisfying these inequalities are called individually rational. The imputation set $\mathrm{I}(v)$ consists of all efficient and individually rational allocations:

$$
\mathrm{I}(v)=\left\{x \in \mathbb{R}^{N} \mid \sum_{i \in N} x_{i}=v(N) \text { and } x_{i} \geq v(\{i\}) \text { for all } i \in N\right\} .
$$

The criterium mentioned above could be strengthened by demanding that not only every player, but also every coalition $S \subseteq N$ should receive at least the amount it can obtain by operating on its own, i.e.

$$
\sum_{i \in S} x_{i} \geq v(S) \text { for all } S \subseteq N .
$$

In the following we denote $\sum_{i \in S} x_{i}$ by $x(S)$. The core consists of all efficient allocations satisfying the above inequalities:

$$
\text { Core }(v)=\left\{x \in \mathbb{R}^{N} \mid x(N)=v(N) \text { and } x(S) \geq v(S) \text { for all } S \subseteq N\right\} .
$$

Core allocations have the property that no coalition can improve its situation by splitting off from the grand coalition $N$. Hence, if core allocations exist for a game $v$, then it is very likely that the grand coalition $N$ forms, and that the profit $v(N)$ will be allocated according to some vector in Core $(v)$.

In the earlier given example, where the players Alfred, Bert and Chris try to distribute the 60 dollars they can obtain, the core is empty. No matter how the money is divided among the players, there will always be a coalition which receives less than it could ubtain by working alone. This coalition has a plausible threat to stop cooperation in the grand coalition $N$. Such a situation can prevent the formation of the coalition $N$.

The core can be viewed as a solution concept in the sense that it assigns to every game a set of (reasonable) allocations. Other well-known solution concepts are the bargaining set (Aumann and Maschler [3]), the kernel (Davis and Maschler [13]) and the prekernel (Maschler, Peleg and Shapley[33]). The fact that these solution concepts do not assign a single allocation to a game, but a (possibly empty) set of allocations, can be regarded as a disadvantage. Therefore, there is also some interest in solution concepts which assign to every game exactly one allocation. Among these, the Shapley value (Shapley[50]), the prenucleolus and the nucleolus (Schmeidler[48]), and the $\tau$-value ( $\mathrm{Tijs}[60]$ ) are the best known. An advantage of the prenucleolus and the nucleolus is that they always lie in the core of the game if the core is non-empty. Their definition is as follows. 
Let $v$ be a cooperative game with player set $N$. For an allocation $x \in \mathbb{R}^{N}$ and $S \subseteq N$ the value $v(S)-x(S)$ is called the excess of coalition $S$ at $x$. Intuitively, the excess of coalition $S$ at $x$ can be interpreted as a measure of discontent of $S$ with $x$. Let $\theta(x)$ be the vector of all excesses at $x(S \subseteq N)$ arranged in order of non-increasing magnitude. The prenucleolus is defined as the set of vectors in $\mathbb{R}^{N}$ which lexicographically minimize the function $\theta(x)$ over the set of efficient allocations. The nucleolus is defined as the set. of vectors in $\mathbb{R}^{N}$ which lexicographically minimize the function $\theta(x)$ over the set of imputations $\mathrm{I}(v)$.

The motivation of these definitions is that the coalition who is least content should be helped as much as possible, so the maximal excess should be minimized. Having done this, we turn to the coalition with second maximal excess, and help this coalition as much as possible, without changing the excess of the first coalition. Then we proceed with the third maximal excess, etc. In the definition of the nucleolus we also keep in mind that the outcome of this process should be individually rational. Notice that the prenucleolus and the nucleolus are defined as set valued solution concepts. However, in case of the prenucleolus, this set turns out to consist of one single point. The same is true for the nucleolus, provided that $\mathrm{I}(v)$ is non-empty (see Schmeidler[48]). We shall denote the prenucleolus of a game $v$ by $P \mathcal{N}(v)$ and the nucleolus by $\mathcal{N}(v)$.

The definitions of the above mentioned solution concepts are all motivated by some intuitive sense of fairness, perhaps one more convincing than the other. But in all cases, the plausibility of such a motivation depends on the assumption that the worth $v(S)$ of a coalition is interpreted as a profit and not as a cost. Therefore, each solution concept has its counterpart, which is applicable in case $v(S)$ should be interpreted as a cost. We shall give the definitions of the counterparts of the imputation set, the core, and the (pre)nucleolus respectively.

The anti-imputation set of a game $v$ is defined as

$$
\operatorname{AI}(v)=\left\{x \in \mathbb{R}^{N} \mid \sum_{i \in N} x_{i}=v(N) \text { and } x_{i} \leq v(\{i\}) \text { for all } i \in N\right\} .
$$

The anti-core of $v$ is defined as

$$
\text { ACore }(v)=\left\{x \in \mathbb{R}^{N} \mid x(N)=v(N) \text { and } x(S) \leq v(S) \text { for all } S \subseteq N\right\} .
$$

Also the prenucleolus and the nucleclus have their counterpart in the socalled anti-prenucleolus and anti-nucleolus. In case the values $v(S)$ are interpreted as costs rather than savings, the excesses at $x$ can be interpreted 
as a measure of content with $x$. Therefore, it is more natural to arrange the excesses in non-decreasing order, and to maximize this vector lexicographically. In case of the anti-prenucleolus the maximization is performed over the set of efficient allocations, in case of the nucleolus the maximization is performed over the anti-imputation set. Anti-prenucleolus and anti-nucleolus are denoted respectively by $A P N(v)$ and $A N(v)$.

With a game $v$ one can associate its so-called dual game $v^{*}$, which is defined by $v^{*}(S)=v(N)-v(N \backslash S)$ for all $S \subseteq N$. The term dual becomes clear by observing that $\left(v^{*}\right)^{*}=v$. We have the following relationships between a game $v$ and its dual.

- $\operatorname{Core}(v)=\operatorname{ACore}\left(v^{*}\right)$.

- $P N(v)=A P N\left(v^{*}\right)$.

Unfortunately, it is not true in general that $\mathcal{N}(v)=A \mathcal{N}\left(v^{*}\right)$. However, when $\operatorname{Core}(v) \neq \emptyset$, the nucleolus and the anti-nucleolus coincide, so in that situation we have $\mathcal{N}(v)=A \mathcal{N}\left(v^{*}\right)$.

In this monograph we consider various classes of cooperative games, such as traveling salesman games, minimum cost spanning tree games and assignment games. As the names suggest, each of these games is closely related to a well-known combinatorial optimization problem. The general idea behind this is the following. A group of people, denoted by $N$, work together in some project. Each subset $S \subseteq N$ can form a coalition, and if they do so, they will try to maximize their profit or minimize their cost in this project. The set of possible actions that coalition $S$ can take corresponds to the set of feasible solutions of some combinatorial optimization problem associated with $S$. The worth $v(S)$ is taken to be the profit or cost which results from an optimal action or perhaps from some specified heuristic action of $S$. In this monograph we investigate the properties of some cooperative games, which are related in this way to a combinatorial optimization problem. We have mainly concentrated on the structure of the core and on the nucleolus of these games.

Often the combinatorial optimization problem associated with a coalition. $S$ is defined on a weighted graph. For an overview of literature on games in which graphs play this role, we refer to Sharkey[53].

The outline of this monograph is as follows. In chapter 2 we consider games for which the characteristic function is defined on only a subset $B \subseteq 2^{N}$ of coalitions. The interpretation of the collection $B$ is that of the collection of formable coalitions. The motivation to consider such games is 
that in some situations communication between players may be hindered, in which case only coalitions need to be considered for which full communication between players is possible. Models like these are studied in Myerson[38] and Owen[44]. The reason to consider these games in this monograph is that the core and the nucleolus of some interesting classes of games only depend on coalitions in some suitably chosen collection $B$, not because cooperation between players is restricted, but simply because of the inherent structure of these games.

In chapter 3 we consider games which are associated to the traveling salesman problem. Chapter $\mathbf{4}$ deals with games which are in some way related to the minimum cost spanning tree problem, and chapter 5 is devoted to games arising from the (multi-dimensional) assignment problem. In all of these chapters we pay special attention to the core (or anti-core in a cost situation) of these games. In some cases we also investigate the nucleolus. The structures we discovered in these chapters led us to the more general question of how a certain structure in the characteristic function of a game influences the structure of the core. In chapter 6 we establish relationships between properties of the characteristic function and the number of extreme. elements of the core. 


\section{Chapter 2}

\section{Games with restricted cooperation}

\subsection{Restricted cores of games}

In this chapter we consider cooperative games $(N, v)$ in which there is a restriction on the formation of coalitions. Situations like these are described in Myerson[38] and Owen[44], where cooperation between players is only possible if there exists a communication link between them. Let us denote the collection of formable coalitions by $B \subseteq 2^{N}$. In this situation it is natural to take only coalitions in $B$ into consideration in the definition of the core. The $B$-restricted core is defined as

$$
\operatorname{Core}(B, v)=\left\{x \in \mathbb{R}^{N} \mid x(N)=v(N) \text { and } x(B) \geq v(B) \text { for all } B \in B\right\} \text {. }
$$

The B-restricted core is also investigated by Faigle[16]. In that paper, the concept of balancedness for games with a limited set of formable coalitions is generalized, and a generalization of the well-known Shapley-Bondareva theorem is derived. Also, the concept of convexity is generalized. These general convex games may have an empty core, but obviously necessary conditions turn out to be sufficient to guarantee the non-emptiness of the B-restricted core.

In contrast with the unrestricted core, the restricted core is not necessarily bounded. In Derks and Reijnierse[15] necessary and sufficient, conditions on $B$ are given, such that the $B$-restricted core is bounded.

Notice that core restrictions may be redundant. In a game $v$ a coalition 
$S$ is said to be essential if

$$
v(S)>\sum_{T \in T} v(T) \text { for each non-trivial partition } T \text { of } S,
$$

where a partition is called non-trivial if it contains at least two non-empty sets. Remark that all 1-person coalitions are essential. The set of essential coalitions is denoted by $\varepsilon(v)$. Essential coalitions are the only coalitions which are needed in the description of the core. This is seen as follows. Let $S$ be a coalition which is not essential. Then there exists a partition $T$ of $S$ into essential coalitions such that $v(S) \leq \sum_{T \in T} v(T)$. When we sum up the restrictions corresponding to the coalitions in $T$, we find

$$
x(S)=\sum_{T \in T} x(T) \geq \sum_{T \in T} v(T) \geq v(S) .
$$

Hence, the restriction corresponding to coalition $S$ is implied by the restrictions corresponding to the coalitions in $\tau$. It follows that

$$
\operatorname{Core}(\varepsilon(v), v)=\operatorname{Core}(v) .
$$

The set of essential coalitions can be a considerably smaller set than $2^{N}$. In this case it is clearly an advantage that one only has to take essential coalitions into consideration.

\subsection{Partitioning games}

Throughout the remainder of this chapter it is assumed that the collection $B$ contains all 1-person coalitions as well as the grand coalition $N$.

Kaneko and Wooders[27] introduced partitioning games. Let $v$ be a game with player set $N$ and let $B \subseteq 2^{N}$. If a collection $T \subseteq B$ forms a partition of $S \subseteq N$, then $T$ is called a $B$-partition of $S$ and $\sum_{T \in T} v(T)$ is called the value of $T$. A $B$-partition $T$ of $S$ with maximal value is called a maximal $B$-partition of $S$. The game $v$ is called a partitioning game induced by $B$ if $v(S)$ equals the value of a maximal B-partition of $S$ for all $S \subseteq N$. The fact that all 1-person coalitions are members of $B$ ensures that every coalition has at least one $B$-partition. The class of partitioning games induced by $B$ is denoted by $\operatorname{GS}(N, B)$. The notation GS is due to Kaneko and Wooders, and stands for games with sidepayments.

For any $S \notin B$ the 8-partition $\tau$ for which $v(S)=\sum_{T \in T} v(T)$ must be a non-trivial partition. Hence, $S$ cannot be essential. We conclude that 
only coalitions in $B$ can be essential for games in $\operatorname{GS}(N, B)$. Therefore, the B-restricted core coincides with the core.

Kaneko and Wooders prove that all games in $\operatorname{GS}(N, B)$ have a non-empty core if and only if every extreme solution of the system

$$
\sum_{B: i \in B \in B} \lambda_{B}=1 \text { for all } i \in N, \lambda_{B} \geq 0 \text { for all } B \in B
$$

is integral. This theorem is strongly related to the notion of perfect matrices.

Let $A$ be a matrix whose entries are all 0 or 1, having no zero column. The matrix $A$ is called perfect if every extreme solution of the system $A x \leq$ $1, x \geq 0$ is integral. See e.g. Golumbic[21]. Let us index the members of $B$, except the 1-person coalitions and the empty coalition, and define the matrix $A^{B}$ by

$$
A_{i j}^{8}= \begin{cases}1 & \text { if } i \in B_{j} \\ 0 & \text { otherwise. }\end{cases}
$$

We call the collection $B$ perfect if the matrix $A^{B}$ is perfect.

Observe that Kaneko and Wooders' theorem is equivalent to saying that all games in $\operatorname{GS}(N, B)$ have a non-empty core if and only if the collection $B$ is perfect. In order to prove this theorem it is convenient to formulate the search for a core element as a linear programming problem. Define the core problem or $\operatorname{CP}(B, v)$ by

$$
\begin{array}{ll}
\min & x(N) \\
\text { subject to } & x(B) \geq v(B) \quad \text { for all } B \in B .
\end{array}
$$

The dual of $\operatorname{CP}(B, v)$ is the fractional partitioning problem $\operatorname{FPP}(B, v)$ defined by

$$
\begin{array}{lll}
\max & \sum_{B \in B} \lambda_{B} v(B) & \\
\text { subject to } & \sum_{B: i \in B} \lambda_{B}=1 & \text { for all } i \in N \\
& \lambda_{B} \geq 0 & \text { for all } B \in B .
\end{array}
$$

Observe that the integer version of $\operatorname{FPP}(B, v)$, is precisely the problem of finding a maximal $B$-partition of $N$.

If the optimal value of $\operatorname{CP}(B, v)$ equals $v(N)$, then the set of optimal solutions of $\operatorname{CP}(B, v)$ is exactly the $B$-restricted core and as we have observed earlier, for games in $\operatorname{GS}(N, B)$ the restricted core coincides with the core. In the following we shall prove Kaneko and Wooder's theorem by showing that the optimal value of both $\operatorname{FPP}(B, v)$ and $\operatorname{CP}(B, v)$ equal $v(N)$ for all $v \in \mathrm{GS}(N, B)$ if and only if $B$ is perfect. We need the following two lemmas. 
Lemma 2.1 Let $B \subseteq 2^{N}$ and let $v: B \rightarrow \mathbb{R}$. For each $S \subseteq N$ we denote the value of a maximal $B$-partition w.r.t. the weight function $v$ by $w(S)$. Then

i) The feasible regions of $C P(B, v)$ and $C P(B, w)$ coincide.

ii) $w \in G S(N, B)$.

Proof: Proof of i): For each $B \in B$ the set $\{B\}$ itself forms a $B$-partition of $B$ with value $v(B)$. Hence $w(B) \geq v(B)$, since $w(B)$ is the value of a maximal $B$-partition. It follows that the feasible region of $\operatorname{CP}(B, w)$ is contained in that of $\operatorname{CP}(B, v)$. Now let $x$ be feasible in $\operatorname{CP}(B, v)$ and let $B \in B$. Choose a $B$-partition $T$ of $B$ such that $w(B)=\sum_{T \in T} v(T)$. It follows that

$$
x(B)=\sum_{T \in T} x(T) \geq \sum_{T \in T} v(T)=w(B) .
$$

So $x$ is also feasible in $\operatorname{CP}(B, w)$ and we conclude that the feasible region of $\operatorname{CP}(B, v)$ is contained in that of $\operatorname{CP}(B, w)$. Hence, the feasible regions coincide.

Proof of ii): First we prove that the function $w$ is superadditive, i.e. $w(U)+w(V) \leq w(U \cup V)$ for all $U, V \subseteq N$ with $U \cap V=\emptyset$. Let $S$ and $T$ be B-partitions of $U$ and $V$ respectively, such that $w(U)=\sum_{S \in S} v(S)$ and $w(V)=\sum_{T \in T} v(T)$. Now, $S \cup T$ is a B-partition of $U \cup V$, so

$$
w(U \cup V) \geq \sum_{S \in S} v(S)+\sum_{T \in T} v(T)=w(U)+w(V) .
$$

We have to prove that for each $S \subseteq N$

$$
w(S)=\max \left\{\sum_{T \in T} w(T) \mid T \text { is a } B \text {-partition of } S\right\} .
$$

From the superadditivity of $w$ we obtain

$$
w(S) \geq \max \left\{\sum_{T \in T} w(T) \mid T \text { is a } B \text {-partition of } S\right\} .
$$

We have seen in i) that $w(B) \geq v(B)$ for all $B \in B$. From this it follows that

$$
\begin{aligned}
w(S) & =\max \left\{\sum_{T \in T} v(T) \mid T \text { is a } B \text {-partition of } S\right\} \\
& \leq \max \left\{\sum_{T \in T} w(T) \mid T \text { is a } B \text {-partition of } S\right\}
\end{aligned}
$$

In Nemhauser and Wolsey[39] (page 536) the following lemma is proved. 
Lemma 2.2 Let $A$ be an $m \times n$ matrix, and let $b \in \mathbb{R}^{n}$. All extreme elements of the polyhedron $A x \leq b$ are integral if and only if $\max \{c x \mid A x \leq b\}$ is integral for all $c \in Z^{n}$ for which this linear program has an optimal solution.

Now we are in a position to prove Kaneko and Wooders' theorem.

Theorem 2.3 The following statements are equivalent.

i. $B$ is perfect.

ii. Every game in $G S(N, B)$ has a non-empty core.

Proof: Suppose that $B$ is perfect and let $v \in \mathrm{GS}(N, B)$. Let $\bar{\lambda}$ be an extreme optimal solution of $\operatorname{FPP}(B, v)$. The perfectness of $B$ ensures that $\bar{\lambda}_{B} \in\{0,1\}$ for all $B \in B$. Let $T=\left\{B \in B \mid \bar{\lambda}_{B}=1\right\}$. Then $T$ forms a $B$-partition of $N$, and since it is the result of a maximization, it must be a maximal B-partition of $N$. Since $v \in \operatorname{GS}(N, B)$, it follows that $\sum_{T \in T} v(T)=v(N)$. We proved that the optimal value of $\operatorname{FPP}(B, v)$ equals $v(N)$ and according to the duality theorem of linear programming it follows that the optimal value of $\operatorname{CP}(B, v)$ is also $v(N)$. We conclude that $\operatorname{Core}(B, v) \neq \theta$, and since $\operatorname{Core}(v)=\operatorname{Core}(B, v)$ for games in $\operatorname{GS}(N, B)$, also Core $(v) \neq \emptyset$.

Now suppose that every game in $\operatorname{GS}(N, B)$ has a non-empty core. We have to prove that $B$ is perfect. According to lemma 2.2 it suffices to show that the optimal value of $\operatorname{FPP}(B, v)$ is integral for every integral valued function $v: B \rightarrow Z$. For each $S \subseteq N$ we define $w(S)$ as the value of a maximal B-partition of $S$ w.r.t. the weight function $v$. According to lemma 2.1 we have $w \in G S(N, B)$. By assumption $w$ has a non-empty core, so we conclude that the value of $\operatorname{CP}(B, w)$ equals $w(N)$. Also according to lemma 2.1, the feasible regions of $\operatorname{CP}(B, v)$ and $\operatorname{CP}(B, w)$ coincide, so also the value of $\mathrm{CP}(B, v)$ equals $w(N)$. According to the duality theorem of linear programming the value of $\operatorname{FPP}(B, v)$ equals $w(N)$ : an integer.

A coalition $S \subseteq N$ is called $B$-stable if

$$
v(S) \geq \sum_{T \in T} v(T) \text { for each B-partition } T \text { of } S \text {. }
$$

A coalition which is $2^{N}$-stable is simply called stable. Using theorem 2.3 one easily proves the following theorem.

Theorem 2.4 Let $v$ be a game with player set $N$ and let $B \subseteq 2^{N}$ be perfect. Then the $B$-restricted core of $v$ is non-empty, if and only if the grand coalition is B-stable. 
Proof: The necessity of B-stability is trivial, so let us prove sufficiency. For each $S \subseteq N$ we define $w(S)$ as the value of a maximal $\boldsymbol{b}$-partition of $S$ w.r.t. the weight function $v$. According to lemma 2.1 we have $w \in \operatorname{GS}(N, B)$. Since $N \in B$ we have $w(N) \geq v(N)$. The $B$-stability of $N$ in $v$ ensures that $w(N) \leq v(N)$. We conclude that $w(N)=v(N)$. From the fact that the feasible regions of $\operatorname{CP}(B, v)$ and $\operatorname{CP}(B, w)$ coincide, it now follows that $\operatorname{Core}(B, v)=\operatorname{Core}(B, w)$. Applying theorem 2.3 to $w \in \operatorname{GS}(N, B)$ we derive

$$
\operatorname{Core}(B, v)=\operatorname{Core}(B, w)=\operatorname{Core}(w) \neq \emptyset .
$$

Our objective is to provide sufficient conditions on $B$, such that the collection perfect. The theory on perfect graphs can guide the search for such sufficient conditions.

\subsection{Perfect graphs and perfect collections}

In a slightly different form, the results in this section can be found in Golumbic[21]. Let $G=(N, E)$ be an undirected graph. A subset $S \subseteq N$ is cailed a cligue if $\{i, j\} \in E$ for all $i, j \in S . S$ is called a maximal clique if it is a clique and if it is not a proper subset of another clique. The clique matrix of $G$ is the $(0,1)$-incidence matrix whose rows correspond to all of the maximal cliques of $G$, and whose columns correspond to the vertices of $G$. The graph $G$ is called perfect if its clique matrix is perfect. This definition of a perfect graph is the one which is also used in Nemhauser and Wolsey[39]. Another definition, purely in graphical terms, is given in Golumbic[21]. It is shown both in Nemhauser and Wolsey and in Golumbic that the two definitions are equivalent.

Let $A$ be an $m \times n(0,1)$-valued matrix. Let $G$ be the graph with vertex set $N=\{1, \ldots, n\}$ and an edge between $i$ and $j$ if the $i$-th and $j$-th column of the matrix $A$ have a 1 at corresponding places. $G$ is called the intersection graph of the matrix $A$. The intersection graph of a collection $B$ is defined as the intersection graph of the matrix $A^{8}$. The following lemma establishes a relationship between a perfect collection and its intersection graph.

Lemma 2.5 If $B \subseteq 2^{N}$ is a perfect collection, then the intersection graph of $B$ is a perfect graph. 
Proof: For any $m \times n$ matrix $M$ let $P(M)=\left\{x \in \mathbb{R}^{n} \mid M x \leq 1, x \geq 0\right\}$ and let $P_{I}(M)=\operatorname{conv}\left\{x \in \mathbb{R}^{n} \mid M x \leq \mathbf{1}, x \geq 0, x\right.$ integral $\}$.

Let $G=(N, E)$ be the intersection graph of $B$. Furthermore, let $A$ denote the matrix $A^{B}$, and let $\bar{A}$ be the clique matrix of $G$. We shall first prove that $P(\bar{A}) \subseteq P(A)$. Let $a$ be a row of the matrix $A$. Obviously, the collection $S=\left\{i \in N \mid a_{i}=1\right\}$ is a clique (not necessarily maximal) in the graph $G$. Let $C$ be a maximal clique in $G$ which contains $S$ as a subset, and define the vector $\bar{a}$ by $\bar{a}_{i}=1$ if $i \in C$ and $\bar{a}_{i}=0$ otherwise. $\bar{a}$ is a row vector of the matrix $\bar{A}$ and we have $\bar{a} \geq a$. Observe that the restriction $a x \leq 1$ is implied by the restriction $\bar{a} x \leq 1$ and the restrictions $x_{i} \geq 0$ for $i \in C \backslash S$. Hence, every restriction in the definition of the polyhedron $P(A)$ is implied by some restrictions in the definition of the polyhedron $P(\bar{A})$. It follows that $P(\bar{A}) \subseteq P(A)$.

Now we shall prove that $P_{I}(A) \subseteq P_{I}(\bar{A})$. Suppose $x \in P_{I}(A)$. Since $A$ is without zero colurnn, it follows that $x \leq 1$, that is, $x$ is a $(0,1)$-vector. Define $S=\left\{i \in N \mid x_{i}=1\right\}$. Suppose $i, j \in S$ are adjacent in $G$. Then some row of $A$, say $a$, has a 1 at places $i$ and $j$. This yields $a x \geq 2$, contradicting the fact that $A x \leq 1$. We conclude that no two elements of $S$ are adjacent in $G$. It also follows that $S$ contains at most one vertex of every (maximal) clique in $G$. This yields $\bar{A} x \leq 1$, and hence $x \in P_{I}(\bar{A})$.

Now we have

$$
P_{I}(\bar{A}) \subseteq P(\bar{A}) \subseteq P(A)=P_{I}(A) \subseteq P_{I}(\bar{A}),
$$

where the first inclusion is trivial, the second and third inclusion were just proven, and the equality follows from the perfectness of the matrix $A$. Hence, we have $P_{I}(\bar{A})=P(\bar{A})$. In other words, $\bar{A}$ is a perfect matrix and consequently $G$ is a perfect graph.

Note that the converse of lemma 2.5 is not true, that is, a collection is not necessarily perfect if its intersection graph is perfect. A simple counterexample is given by the collection $\{\{1,2\},\{1,3\},\{2,3\}\} \subseteq 2^{\{1,2,3\}}$.

A $(0,1)$-matrix $A$ is said to satisfy the Helly property if every row in the clique matrix of the intersection graph of $A$ is a row of the matrix $A$ itself. We say that a collection $B$ satisfies the Helly property if the matrix $A^{B}$ does so. Equivalently, $B$ satisfies the Helly property if for any subcollection. $T \subseteq B$ with $S \cap T \neq \emptyset$ for all $S, T \in T$, we have that $\cap_{T \in T} T \neq \emptyset$.

Lemma 2.6 A perfect collection satisfies the Helly property. 
Proof: Suppose $B \subseteq 2^{N}$ does not satisfy the Helly property. We shall prove that $B$ is not perfect. Let $A$ denote the matrix $A^{B}$. Choose a maximal clique $C$ in the intersection graph of $B$ which does not correspond to a row of the matrix $A$. Observe that $|C| \geq 3$. Let $c$ denote the incidence vector of $C$.

For any row $a$ of the matrix $A$ we have $a \neq c$, so it follows that $a c \leq$ $|C|-1$. Hence, $c /(|C|-1)$ is a non-integral feasible solution of the system $A x \leq 1, x \geq 0$. It suffices to show that $c /(|C|-1)$ cannot be written as a convex combination of integral solutions of this system.

Let $\bar{x}$ be an integral solution of $A x \leq 1, x \geq 0$. For any pair $i, j \in C$ $(i \neq j)$, columns $i$ and $j$ of the matrix $A$ have a 1 at some corresponding place. Hence, $\bar{x}_{i}$ and $\bar{x}_{j}$ cannot both be equal to 1 , since this would yield $a \bar{x} \geq 2$ for some row $a$ of $A$. It follows that $\bar{x} c \leq 1$.

Now suppose $c /(|C|-1)$ can be written as a convex combination of integral solutions, say $c /(|C|-1)=\sum_{i=1}^{k} \lambda_{i} x^{i}$. Then

$$
|C|=c c=(|C|-1) \sum_{i=1}^{k} \lambda_{i} x^{i} c \leq(|C|-1) \sum_{i=1}^{k} \lambda_{i}=|C|-1,
$$

a contradiction. Hence, $c /(|C|-1)$ cannot be written as a convex combination of integral solutions. It follows that $A$ is not a perfect matrix and that the collection $B$ is not perfect.

The combination of the two necessary conditions for a collection to be perfect mentioned in limmas 2.5 and 2.6 turns out to be sufficient.

Theorem 2.7 A collection is perfect if and only if its intersection graph is perfect and if it satisfies the Helly property.

Proof: The 'only if'-part of the theorem follows directly from lemmas 2.5 and 2.6. So let us prove the 'if'-part.

For any $m \times n$ matrix $M$ let $P(M)=\left\{x \in \mathbb{R}^{n} \mid M x \leq 1, x \geq 0\right\}$. Let $B \subseteq 2^{N}$ be a collection whose intersection graph is perfect and which satisfies the Helly property. Let $A$ denote the matrix $A^{B}$, and let $\bar{A}$ denote the clique matrix of the intersection graph of $B$. Since $A$ satisfies the Helly property, every row of $\bar{A}$ is also a row of $A$. Hence, $P(A) \subseteq P(\bar{A})$. In lemma 2.5 we already proved that $P(\bar{A}) \subseteq P(A)$ (this result did not depend on the conditions mentioned in the lemma). It follows that $P(A)=P(\bar{A})$, and since $P(\bar{A})$ is integral, it follows that $P(A)$ is integral too. Thus, $A$ is a perfect matrix, and $B$ is a perfect collection. 
The characterization of perfect collections in theorem 2.7 can be very helpful in proving whether a collection $B$ is perfect or not, since several classes of perfect graphs are known in literature.

In their paper Kaneko and Wooders mention the class of assignment games as an example on which theorem 2.4 can be applied. The collection of essential coalitions of an assignment games satisfies the Helly property, and the intersection graph is bipartite. Indeed, the class of bipartite graphs is a well-known class of perfect graphs (see Nemhauser and Wolsey[39]). However, bipartite graphs are not the only graphs that are known to be perfect. Another important class is the class of triangulated graphs (their definition will follow later). In the next section we give conditions on $B$ such that it satisfies the Helly property and such that the intersection graph of $B$ is triangulated. Hence, a collection satisfying these condition is perfect. Further, we give some interesting classes of games for which the collection of essential coalitions satisfies these conditions. These games have therefore a non-empty core. This shows that the scope of theorem 2.4 is somewhat larger than Kaneko and Wooders suggest.

\subsection{The total dependency property}

A player $i \in N$ is called $B$-dependent if there is a player $j \neq i$ such that for all $B \in B$ with $i \in B$ we have that either $B=\{i\}$ or $j \in B$. Let $x$ be a permutation of the elements of $N$. The collection $B \subseteq 2^{N}$ is said to have the total dependency property w.r.t. $\pi$ (TD-property) if for each $k \in\{1,2, \ldots, n\}$, player $\pi_{k}$ is a $B_{k}^{\pi}$-dependent player, where $B_{k}^{\pi}=\left\{B \cap\left\{\pi_{k}, \ldots, \pi_{n}\right\} \mid B \in B\right\}$. When we say that $B \subseteq 2^{N}$ has the TD-property without referring to some permutation, we mean that there exists a permutation $\pi$ such $B$ has the TD-property w.r.t. $\pi$.

Let $G=(N, E)$ be an undirected graph with vertex set $N$ and edge set $E$. A coalition $S \subseteq N$ is said to be connected in $G$ if the graph that results from $G$ by deleting all players outside $S$ is a connected graph.

Theorem 2.8 The following statements are equivalent.

i) The collection $B$ has the TD-property.

ii) There exists a forest $\Gamma=(N, E)$ such that all members of $B$ are connected in $\Gamma$. 
Proof: ii) $\Rightarrow$ i). We say that a player $i \in N$ is a leaf of $\Gamma$ if there is at most one $j \neq i$ such that $\{i, j\} \in E$. Forests always contain at least one leaf. Choose a leaf in $\Gamma=\Gamma_{1}$ and give this player number 1 . Now, remove this player from $\Gamma_{1}$ and denote the resulting forest by $\Gamma_{2}$. Again, choose a, leaf in $\Gamma_{2}$ and give this player number 2 . Proceed until all players are renumbered, such that for each $k \in N, k$ is a leaf in the forest $\Gamma_{\boldsymbol{k}}$, which results from $\Gamma$ by deleting the players with index less than $k$ from it. If there is no player adjacent to $k$ in the forest $\Gamma_{k}$, then choose $l>k$ arbitrarily. Otherwise, let $l$ be the unique player with $l>k$ and $\{k, l\} \in E$. Let $B \in B$ with $k \in B$. $B$ is connected in $\Gamma$ and hence, $B \cap\{k, \ldots, n\}$ is connected in $\Gamma_{k}$. Then $B \cap\{k, \ldots, n\}$ must either be equal to the singleton $\{k\}$, or it must contain player $l$ as well. It follows that $k$ is dependent in $B_{k}$ and that $B$ has the TD-property.

i) $\Rightarrow$ ii). Suppose that $B$ has the TD-property. We shall construct a forest such that all members of $B$ are connected in this forest. In fact, this forest will be connected, that is, it will be a tree.

Assume without loss of generality that $B$ has the TD-property w.r.t. the permutation $(1,2, \ldots, n)$. For each $k \in N \backslash\{n\}$ let $p(k)$ denote a, player, such that $k$ is dependent on $p(k)$ in the collection $B_{k}=\{B \cap\{k, \ldots, n\} \mid B \in B\}$. Define the edge set $E$ by

$$
E=\{\{k, p(k) \mid k \in N \backslash\{n\}\}
$$

and define $\Gamma=(N, E)$. Let $B \in B$. We shall prove that $B$ is connected in $\Gamma$. Let $k, l \in B$. Consider the following algorithm to find a path between $k$ and $l$.

$$
\begin{aligned}
& \quad i=0 ; k_{0}=k ; l_{0}=l ; \\
& \text { while } k_{i} \neq l_{i} \\
& \underline{\text { do }} \\
& \quad \text { if } k_{i}<l_{i} \\
& \quad \underline{\text { then }} k_{i+1}=p\left(k_{i}\right) ; l_{i+1}=l_{i} ; \\
& \quad \text { else } k_{i+1}=k_{i} ; l_{i+1}=p\left(l_{i}\right) ; \\
& \quad \underline{\text { i }} \\
& \quad i=i+1 ;
\end{aligned}
$$

The TD-property ensures that all vertices which are generated by the algorithm are elements of $B$. Observe that $p(k)>k$ for all $k \in N \backslash\{n\}$. Hence, 
the sequence $k_{0}+l_{0}, k_{1}+l_{1}, \ldots$ is strictly increasing. Since $k_{0}+l_{0} \geq 2$ and $k_{i}+l_{i} \leq 2 n$ for all $i$, it follows that the algorithm terminates in at most $2 n$ steps. When the algorithm terminates, the sequences $k_{0}, k_{1}, \ldots$ and $l_{0}, l_{1}, \ldots$ form a path between $k$ and $l$ which uses only vertices in $B$ and edges in $E$. It follows that all coalitions in $B$ are connected in the graph $\Gamma$. The same algorithm can be used to show that $N$ is connected in $\Gamma$. In other words, $\Gamma$ is connected. From this, together with the fact that $\Gamma$ has only $n-1$ edges, we conclude that $\Gamma$ is a tree and thus, all coalitions in $B$ are connected in a tree.

A graph is called triangulated if every cycle of length strictly greater than 3 possesses an edge joining two non-sequential vertices in the cycle. Such an edge is called a chord. Therefore, triangulated graphs are sometimes called chordal graphs.

Triangulated graphs are well-known to be perfect (see e.g. Golumbic[21]). This result can help us to prove that a collection $B$ which possesses the TDproperty is perfect. In Golumbic it is proved that a collection $B$ which satisfies property ii) of theorem 2.8 satisfies the Helly property, and that the intersection graph of such a collection is triangulated. Hence, $B$ is perfect.

We prefer to include an alternative proof, because our proof provides a constructive way to find elements in the $B$-restricted core if $B$ has the TD-property. Consider again the problems $\operatorname{FPP}(B, v)$ and $\operatorname{CP}(B, v)$. Our objective is to show that $\operatorname{FPP}(B, v)$ and $\operatorname{CP}(B, v)$ are easily solved if $B$ has the TD-property. In the remainder of this section we assume that $B$ has the TD-property w.r.t. the permutation $(1,2, \ldots, n)$.

Define the vector $y^{B}$ recursively by

$$
y_{k}^{B}=\max \left\{v(B)-y^{B}(B \backslash k) \mid k \in B \in B \text { and } B \subseteq\{1, \ldots, k\}\right\} .
$$

The requirement that all 1-person coalitions are contained in $B$ ensures that $y^{B}$ is well-defined. In a game theoretic context the vector $y^{B}$ can be interpreted as follows. Suppose the players of $N$ enter a room in the order $1,2, \ldots, n$. When player $k$ enters the room he is allowed to form a coalition with any subset of players who are already in the room. Say he forms a coalition together with the players in $S \subseteq\{1, \ldots, k-1\}$. The payoff for player $k$ will then be the value of the newly formed coalition minus the payoff for the players in $S$, i.e. $v(S \cup\{k\})-y^{B}(S)$. The most rational choice for player $k$ is to join a coalition $S$ which will give him a maximum payoff. If player $k$ is allowed to take only coalitions in $B$ into consideration, this procedure: 
yields exactly the vector $y^{B}$. Observe that $y^{B}$ can be computed in $O(n|B|)$ elementary operations. In the following we shall omit the superscript $B$ in $y^{B}$ whenever the notation tends to become too complex.

\section{Lemma $2.9 y^{B}$ is a feasible solution of $C P(B, v)$.}

Proof : Let $B \in B$ and let $k$ be the player with maximum index in $B$. From the definition of $y$ it follows that $y_{k} \geq v(B)-y(B \backslash\{k\})$ and therefore, $y(B) \geq v(B)$.

Lemma 2.9 expresses that we have some reason to regard $y^{8}$ as a candidate for an optimal solution of $\operatorname{CP}(B, v)$. If $B \subseteq N$ has the TD-property, then this is indeed the case.

Theorem 2.10 If $B \subseteq 2^{N}$ has the TD-property, then $y^{B}$ is an optimal solution of $C P(B, v)$, and $F P P(B, v)$ has an optimal integral solution.

Proof: According to the duality theorem of linear programming it suffices to construct a partition of $N$ with value $y(N)$. Let $B_{k}$ denote a maximizing coalition in

$$
\max \{v(B)-y(B \backslash k) \mid k \in B \in B \text { and } B \subseteq\{1, \ldots, k\}\} .
$$

Furthermore, define the collections $\mathcal{C}_{k}(k \in N)$, recursively as follows.

$$
\begin{array}{ll}
C_{n}=\left\{B_{n}\right\}, & \\
C_{k}= \begin{cases}C_{k+1} & \text { if } k \text { is contained in one of the elements of } C_{k+1} \\
C_{k+1} \cup\left\{B_{k}\right\} & \text { otherwise. }\end{cases}
\end{array}
$$

We shall prove by induction that the members of $C_{k}$ are mutually disjoint for all $k \in N$. The induction hypothesis is trivially true for $n$. Let $k<n$ and suppose that the induction hypothesis is true for $k+1$. If player $k$ is an element of one of the coalitions in $C_{k+1}$, then $C_{k}=C_{k+1}$. In this case the induction hypothesis is trivially true for $k$ also. If player $k$ is not an element of any of the coalitions in $C_{k+1}$, then $C_{k}=C_{k+1} \cup\left\{B_{k}\right\}$. We have to prove then that $B_{k} \cap B_{i}=\emptyset$ for all $B_{i} \in C_{k}$. In order to prove this, suppose that $j$ is an element of both $B_{k}$ and $B_{i}$ for some $B_{i} \in C_{k+1}$. We choose $j$ maximal. From $B_{k} \subseteq\{1, \ldots, k\}$ and $k \notin B_{i}$, it follows that $j<k$. Player $j$ is a dependent player in the collection $B_{j}=\{B \cap\{j, \ldots, n\} \mid B \in B\}$. Say that he is dependent on player $l$ in this collection. $B_{k} \cap\{j, \ldots, n\} \neq\{j\}$, since $k \in B_{k}$. Therefore $l \in B_{k}$. Analogously, one proves that $l \in B_{i}$. This 
contradicts the maximality of $j$ and it proves that the induction hypothesis is true for $k$ also. We conclude that the induction hypothesis is true for all $k \in N$. Applying this result for $k=1$, we find that $C_{1}$ forms a partition of $N$. For all $B \in C_{1}$ we have $y(B)=v(B)$. Therefore,

$$
y(N)=\sum_{B \in C_{1}} y(B)=\sum_{B \in C_{1}} v(B) .
$$

Corollary 2.11 If $B \subseteq 2^{N}$ has the TD-property, then $B$ is perfect.

Proof: This follows easily from the fact that $\operatorname{FPP}(B, v)$ has an optimal integral solution, regardless of the characteristic function $v$.

Combining theorem 2.4 , theorem 2.10 and corollary 2.11 we obtain the following theorem.

Theorem 2.12 Let $(N, v)$ be a game in which the grand coalition is B-stable for some collection $B \subseteq 2^{N}$. If $B$ has the TD-Property, then $y^{B}$ defined by (2.2) is an extreme element of the B-restricted core of $v$.

Proof: It follows immediately from theorem 2.4 and corollary 2.11 that $\operatorname{Core}(B, v) \neq \emptyset$. As observed in section 2.2 , the set of optimal solutions of $\operatorname{CP}(B, v)$ is then equal to $\operatorname{Core}(B, v)$, so according to theorem 2.10 we have $y^{B} \in \operatorname{Core}(B, v)$.

To prove the extremality of $y^{B}$ observe that $y\left(B_{k}\right)=v\left(B_{k}\right)$ for all $k \in N$, where the sets $B_{k}$ are defined as in theorem 2.4. Let $A$ be the matrix whose $k$-th column is the incidence vector $1_{B_{k}}$. For all $k \in N$ we have $k \in B_{k}$ and $l \notin B_{k}$ for $l>k$. Hence, $A$ is an upper triangular matrix with ones on the diagonal. It follows that $A$ is non-singular, or equivalently, the $n$ incidence vectors $1_{B_{k}}(k \in N)$ are linearly independent. The extremality of $y^{B}$ follows.

Using the observation that the $\mathcal{E}(v)$-restricted core of a game $v$ coincides with its unrestricted core, we find the following corollary of theorem 2.12.

Corollary 2.13 Let $v$ be a game for which the grand coalition is stable, and suppose that the collection of essential coalitions has the TD-Property. Then $v$ has a non-empty core. 
Proof: Define $B=\varepsilon(v) \cup\{N\}$. The addition of $N$ to the set of essential coalitions does not disturb the TD-property. Now apply theorem 2.12 to prove that $y^{B}$ is an element of the B-restricted core. Furthermore, observe that the restricted core coincides with the unrestricted core, so that $y^{B} \in \operatorname{Core}(v)$.

In the following we give some classes of games satisfying the conditions of corollary 2.13, and therefore also satisfying the conditions of theorem 2.4.

A game $v$ is called a simple game if $v(S)=0$ or $v(S)=1$ for all $S \subseteq N$. A player $i \in N$ in such a game is called a veto player if $v(S)=0$ for all $S \subseteq N$ with $i \notin S$. Let $v$ be a simple game which has a veto player, say $i$, and for which $v(N)=1$. Then the grand coalition is stable, and all essential coalitions are connected in the star tree pictured in figure 2.1. Hence, corollary 2.13 can be applied here to prove that the core is non-empty.

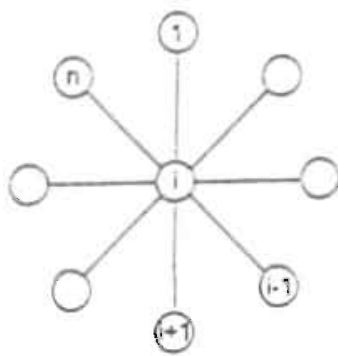

figure 2.1

In Curiel, Pederzoli and Tijs[11] the class of sequencing games is considered. In their paper they show that all essential coalitions are connected in a path (see figure 2.2 below). Also, the grand coalition is stable for such a game. Therefore, corollary 2.13 can also be applied here to prove that sequencing games have a non-empty core. However, in [11] the stronger property is derived that sequencing games are convex.

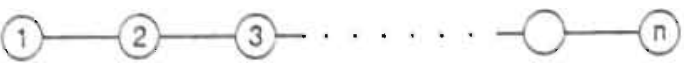

figure 2.2 
In chapter 3 we consider the class of routing games. For these games the value of a coalition is interpreted as a cost. Therefore, we are interested in the anti-core of such a game. In chapter 3 we consider the dual of a routing game and we show that all essential coalitions of the dual are connected in a path. The grand coalition in the dual game is not necessarily stable. Applying corollary $\mathbf{2 . 1 3}$ we find that the dual of a routing game has a nonempty core if and only the grand coalition is stable in the dual game, and consequently the routing game itself has a non-empty anti-core in this case.

Granot and Huberman[24] treat the class of minimum cost spanning tree games. In their paper they prove the so called minimum excess coalition structure theorem. This theorem implies that all essential coalitions of the dual of a minimum cost spanning tree game are connected in a forest whose node set is the player set. Also, the grand coalition in the dual is stable. Again, corollary 2.13 can be applied to prove that minimum cost spanning tree games have a non-empty anti-core. In chapter 4 we use corollary 2.13 to further investigate the structure of the anti-core of minimum cost spanning tree games.

\subsection{The B-prenucleolus}

Let $(N, v)$ be a game and let $B \subseteq 2^{N}$ be a collection of formable coalitions. Recall that the prenucleolus of a game is the outcome of a lexicographic minimization which involves all coalitions. In case only coalitions in $B$ are formable, it is natural to demand that coalitions outside $B$ should not take part in this lexicographic minimization. This leads to the following definition of the B-prenucleolus $P \mathcal{N}(B, v)$ of a game $v$.

For a vector $x \in \mathbb{R}^{N}$ and $S \subseteq N$ the value $v(S)-x(S)$ is called the excess of coalition $S$ at $x$. Let $\theta(B, x)$ be the vector of all excesses at $x$ $(S \in B)$ arranged in order of non-increasing magnitude. The $B$-prenucleolus is defined as the set of vectors in $\mathbb{R}^{N}$ which lexicographically minimize the vector $\theta(B, x)$ over the set of efficient vectors. Analogously, we define the $B$-nucleolus as the set of vectors in $\mathbb{R}^{N}$ which lexicographically minimize the vector $\theta(B, x)$ over the imputation set $\mathrm{I}(v)$.

In Maschler, Potters and Tijs[34] also a generalization of the nucleolus is considered. Their generalization extends to non-transferable utility games and non-cooperative games as well. For transferable utility games, their definitions and ours coincide. In Maschler et al. an axiomatization of the general nucleolus is given. In this section we shall describe how a general 
purpose algorithm for computing the prenucleolus of a game (transferable utility) should be adjusted when we want to compute the B-prenucleolus instead. A similar adjustment can be made for the B-nucleolus. However, the additional constraint that the outcome should lie in the imputation set complicates things somewhat for the B-nucleolus. For our purposes it will be sufficient to have an algorithm for the prenucleolus, since we will apply the algorithm only on games with a non-empty core, in which case the nucleolus and the prenucleolus coincide. In section 2.6 we shall see that the adjusted algorithm works particularly well when $B$ has the TD-property.

Let us now describe an algorithm for computing the $B$-prenucleolus. It is a straightforward adjustment of an algorithm for computing the prenucleolus as it was presented by Maschler, Peleg and Shapley[33]. The algorithm computes the $B$-prenucleolus by solving a sequence of linear programs.

First assume that $\operatorname{Core}(B, v) \neq \emptyset$. Then there exists a collection $A$ such that $\{N\} \subseteq A \subseteq B$ and such that $x(S)=v(S)$ for all $x \in \operatorname{Core}(B, v)$ and all $S \in A$. Choose a collection $A$ with these properties. If $\operatorname{Core}(B, v)=\emptyset$, then choose $A=\{N\}$. As a first step in finding the $B$-prenucleolus of the game $v$ the following linear program is solved.

$$
\begin{aligned}
& \mathcal{Q}(v, B, A): \text { Minimize } t \\
& \text { subject to } x(S)=v(S) \quad S \in \mathcal{A} \\
& x(S)+t \geq v(S) \quad S \in B \backslash A .
\end{aligned}
$$

Clearly, problem $\mathcal{Q}(v, B, A)$ is feasible and the optimal value is bounded. Observe that $P \mathcal{N}(B, v)$ is a set of feasible solutions of $Q(v, B, A)$. If $A=\{N\}$ this is obvious, since then the set of feasible solutions is the set of efficient vectors, and by definition $P \mathcal{N}(B, v)$ lies in it. If $A \neq\{N\}$ then $\operatorname{Core}(B, v) \neq$ $\emptyset$, and $\operatorname{Core}(B, v)$ is contained in the set of feasible solutions. For $x \in$ $\operatorname{Core}(B, v)$ the vector of excesses is non-positive, while for $x \notin \operatorname{Core}(B, v)$ at least one excess is strictly positive. It follows that $P \mathcal{N}(B, v) \subseteq \operatorname{Core}(B, v)$. Later we shall see that $P \mathcal{N}(B, v)$ is even a set of timal solutions of problem $Q(v, B, A)$.

Let $\bar{t}$ be the optimal value of $\mathcal{Q}(v, B, A)$. There exists a non-empty collection $\bar{A} \subseteq B \backslash A$, such that $x(S)+\bar{t}=v(S)$ for all optimal solutions $(x, \bar{t})$ of $\mathcal{Q}(v, B, A)$ and $S \in \bar{A}$. To make this clear, suppose that for all $S \in B \backslash A$ there exists an optimal solution $\left(x_{S}, \bar{t}\right)$, such that $x_{S}(S)+\bar{t}>v(S)$. Define $\bar{x}=\sum_{S \in B \backslash A} x_{S} /|B \backslash A|$. Then $(\bar{x}, \bar{t})$ is an optimal solution satisfying $\bar{x}(S)+\bar{t}>v(S)$ for all $S \in B \backslash A$. This contradicts the minimality of $\bar{t}$.

The following theorem explains why the $B$-prenucleolus can be computed by solving a sequence of linear programs of the type $\mathcal{Q}\left(v_{i}, B, A_{i}\right)$, 
$(i=1,2, \ldots)$. In the proof we use the notation $x>y$ to denote that the vector $x$ is lexicographically greater than $y$, i.e. $x \neq y$ and the first non-zero coordinate of the vector $x-y$ is positive.

Theorem 2.14 Let $\bar{t}$ be the optimal value of problem $Q(v, B, A)$, and let $\bar{A}$ be a non-empty subcollection of $B \backslash A$ such that $x(S)+\bar{t}=v(S)$ for all optimal solutions $(x, \bar{t})$ and all $S \in \bar{A}$. Furthermore, define the game $w$ by

$$
w(S)= \begin{cases}v(S) & \text { if } S \in A \\ v(S)-\bar{t} & \text { if } S \in B \backslash A .\end{cases}
$$

Then
i) $\operatorname{Core}(B, w) \neq \emptyset$,
ii) $x(S)=w(S)$ for all $x \in \operatorname{Core}(B, w)$ and $S \in A \cup \bar{A}$, and iii) $P \mathcal{N}(B, w)=P \mathcal{N}(B, v)$.

Proof: Proof of i): Choose $\bar{x}$ such that $(\bar{t}, \bar{x})$ is an optimal solution of problem $\mathcal{Q}(v, B, A)$. For $S \in A$ we have $\bar{x}(S)=v(S)=w(S)$, and for $S \in$ $B \backslash A$ we have $\bar{x}(S) \geq v(S)-\bar{t}=w(S)$. Furthermore, $\bar{x}(N)=v(N)=w(N)$, so we conclude that $\bar{x} \in \operatorname{Core}(B, w)$. This proves i), and it also follows that Core $(B, w)$ is a subset of the set of optimal solutions of $Q(v, B, A)$.

Proof of ii): Let $x \in \operatorname{Core}(B, w)$. For $S \in A$ we have $x(S) \geq w(S)=v(S)$ and for $S \in B \backslash A$ we have $x(S)+\bar{t} \geq w(S)+\bar{t}=v(S)$. Hence, $(\bar{t}, x)$ is an optimal solution of problem $\mathcal{Q}(v, B, A)$. It follows that $x(S)=v(S)-\bar{t}=$ $w(S)$ for all $S \in \bar{A}$ and $x(S)=v(S)=w(S)$ for all $S \in A$. This proves ii), and it also follows that $\operatorname{Core}(B, w)$ contains the set of optimal solutions of $\mathcal{Q}(v, B, A)$ as a subset.

Proof of iii): Let $\theta_{v}(x)$ denote the vector of excesses at $x$ for coalitions in $B$ arranged in non-increasing order in the game $v$. Let $\theta_{w}(x)$ be defined analogously.

We have proved that $\operatorname{Core}(B, w) \neq \emptyset$, so we have $P \mathcal{N}(B, w) \subseteq \operatorname{Core}(B, w)$. We shall prove first that also $P N(B, v) \subseteq \operatorname{Core}(B, w)$. Since we have already observed that $P \mathcal{N}(B, v)$ is a set of feasible solutions of $Q(v, B, A)$, it suffices to show that $\theta_{v}(y) \succ \theta_{v}(x)$, for an optimal solution $x$ of $Q(v, B, A)$, and a feasible but not optimal solution $y$. The vector $\theta_{v}(x)$ has at least $|A|-1$ coordinates equal to 0 , and the remaining coordinates are at most $\bar{t}$. The vector $\theta_{v}(y)$ also has $|A|-1$ coordinates equal to 0 , but at least one of the remaining coordinates exceeds $\bar{t}$. Hence, $\theta_{v}(y) \succ \theta_{v}(x)$. This proves that $P \mathcal{N}(B, v) \subseteq \operatorname{Core}(B, w)$. 
Assume $A=\{N\}$. Then $\theta_{w}(x)=\theta_{v}(x)-\mathbf{1} \bar{t}$ for all $x \in \mathbb{R}^{n}$, where $\mathbf{1}$ denotes the all-one vector in $\mathbb{R}^{|B|-1}$. It follows that

$$
\theta_{w}(x) \succ \theta_{w}(y) \Leftrightarrow \theta_{v}(x) \succ \theta_{v}(y)
$$

for any pair $x, y \in \operatorname{Core}(B, w)$. We conclude that $\mathcal{P N}(B, w)=\mathcal{P} \mathcal{N}(B, v)$ in this case.

Now assume $A \neq\{N\}$. Then $\operatorname{Core}(B, v) \neq \emptyset$ and the optimal value $\bar{t}$ of problem $\mathcal{Q}(v, B, A)$ is non-positive. Hence, we can write $\theta_{w}(x)=\theta_{v}(x)-u \bar{t}$ for all $x \in \operatorname{Core}(B, w)$, where $u$ denotes the vector in $\mathbb{R}^{|B|-1}$ which has its first $|A|-1$ coordinates equal to 0 , and the remaining coordinates equal to 1 . It follows that

$$
\theta_{w}(x) \succ \theta_{w}(y) \Leftrightarrow \theta_{v}(x) \succ \theta_{v}(y)
$$

for any pair $x, y \in \operatorname{Core}(B, w)$. We conclude that $P \mathcal{N}(B, w)=P \mathcal{N}(B, v)$.

Hence, by solving a sequence of linear programs of the type $\mathcal{Q}\left(v_{i}, B, A_{i}\right)$ we obtain a sequence of games $v=v_{1}, v_{2}, \ldots$ and a sequence of collections $A=A_{1}, A_{2}, \ldots$, such that the $B$-prenucleoli of all games $v_{i}$ coincide and such that the cardinality of $A_{i}$ is strictly increasing. Thus, after at most $|B|$ steps the set of solutions of the system

$$
x(S)=v_{i}(S) \text { for all } S \in A_{i}
$$

consists of exactly one point, since $B$ contains all 1-person coalitions. This implies that the $B$-restricted core of $v_{i}$ consists of one point. Since the $B$ prenucleolus is always contained in the $B$-restricted core, this point is the 8 -prenucleolus of $v_{i}$, and consequently of $v$.

Schmeidler[48] proved that the prenucleolus consists of one single point. This is true for the $B$-prenucleolus as well when all 1-person coalitions are contained in $B$. From now on we shall refer to the $B$-prenucleolus as a vector in $\mathbb{R}^{N}$ and not as a subset of $\mathbb{R}^{N}$, since we assume throughout that all 1-person coalitions are contained in $B$.

In section 2.3 we proved that the $B$-restricted core coincides with the core in case $B$ contains all essential coalitions. For the $B$-(pre)nucleolus we have a similar result, expressed in corollary 2.16. In the following theorem we use the notation $1_{S}$ to denote the incidence vector of $S \subseteq N$, i.e. the vector whose $i$-th coordinate is 1 if $i \in S$ and 0 if $i \notin S$.

Theorem 2.15 Let $(N, v)$ be a game and let $B \subseteq 2^{N}$ and let $S \subseteq N$ and $T \subseteq B$ be such that $1_{S}$ can be written as a linear combination of the vectors in $\left\{1_{T} \mid T \in T\right\}$. 
Furthermore, suppose that $e(T, \bar{x}) \geq e(S, \bar{x})$ for all $T \in T$ and that $e(T, \bar{y}) \geq e(S, \bar{y})$ for all $T \in T$, where $\bar{x}$ denotes $\mathcal{N}(B, v)$ and $\bar{y}$ denotes $\mathcal{N}(B \cup\{S\}, v)$. Then $\mathcal{N}(B, v)=\mathcal{N}(B \cup\{S\}, v)$.

Proof: Let $\theta(x)$ be the vector of excesses at $x$ of coalitions in $B \cup\{S\}$, arranged in non-increasing order, and let $\phi(x)$ be the vector of excesses of coalitions in $B$ arranged in non-increasing order. Observe that $\phi(x)$ is obtained simply by omitting the excess of coalition $S$ in $\theta(x)$.

Let $\bar{x}=\mathcal{N}(B, v)$ and let $\bar{y}=\mathcal{N}(B \cup\{S\}, v)$. We shall prove that $\bar{x}=\bar{y}$. Let $k$ be such that $\theta_{k}(\bar{x})=e(S, \bar{x})$, and choose $k$ maximal. Furthermore, let $l$ be such that $\theta_{l}(\bar{y})=e(S, \bar{y})$, and choose $l$ maximal. Let $m=\min (l, k)$.

Observe that $\theta_{i}(\bar{x})=\phi_{i}(\bar{x})$ and $\theta_{i}(\bar{y})=\phi_{i}(\bar{y})$ for $i<m$. So, for $i<m$ we have $\theta_{i}(\bar{x})=\theta_{i}(\bar{y})$, since otherwise we would have either $\theta(\bar{x}) \succ \theta(\bar{y})$ or $\phi(\bar{y}) \succ \phi(\bar{x})$, contradicting the fact that $\bar{x}$ lexicographically minimizes $\phi(x)$ and $\bar{y}$ minimizes $\theta(y)$. We not only have $\theta_{i}(\bar{x})=\theta_{i}(\bar{y})$ for $i<m$, but also the collection of coalitions whose excess at $\bar{x}$ equal $\theta_{i}(\bar{x})$ coincides with the collection of coalitions whose excess at $\bar{y}$ equal $\theta_{i}(\bar{y})$.

To prove this, we define $A_{i}(x)=\left\{B \in B \mid \theta_{i}(x)=e(B, x)\right\}$ for $i=$ $1,2, \ldots$ For convenience, we define $A_{0}(x)=\emptyset$. We shall prove by induction that $A_{i}(\bar{x})=A_{i}(\bar{y})$ for $i=0,1, \ldots, m-1$. Define $z=(\bar{x}+\bar{y}) / 2$. For $i=0$ it is trivial that $A_{0}(z)=A_{0}(\bar{x})=A_{0}(\bar{y})=0$. Assume $A_{i}(z)=A_{i}(\bar{x})=A_{i}(\bar{y})$ for $i=0, \ldots, h-1(h<m)$. Then $\theta_{i}(z)=\theta_{i}(\bar{x})$ for $i=0,1, \ldots, h-1$. Choose $B \in A_{h}(z)$. Then we have

$$
\theta_{h}(z)=e(B, z)=\frac{1}{2} e(B, \bar{x})+\frac{1}{2} e(B, \bar{y}) \leq \frac{1}{2} \theta_{h}(\bar{x})+\frac{1}{2} \cdot \theta_{h}(\bar{y})=\theta_{h}(\bar{y}) .
$$

If this inequality were strict then $\theta(z)$ would be lexicographically less than $\theta(\bar{y})$. Since this would be a contradiction, we conclude that $e(B, \bar{y})=\theta_{h}(\bar{y})$ and hence, $B \in \mathcal{A}_{h}(\bar{y})$. It follows that $\mathcal{A}_{h}(z) \subseteq A_{h}(\bar{y})$. If $A_{h}(z)$ were a proper subset of $A_{h}(\bar{y})$, then again this would imply that $\theta(z)$ is lexicographically less than $\theta(\bar{y})$, a contradiction. We conclude that $A_{h}(z)=A_{h}(\bar{y})$. Analogously, one shows that $A_{h}(z)=A_{h}(\bar{x})$, and hence $A_{h}(\bar{x})=A_{h}(\bar{y})=A_{h}(z)$.

Since $e(T, \bar{x}) \geq e(S, \bar{x})$ for all $T \in T$, it follows that each $T \in T$ is a member of one of the coalitions $A_{1}(\bar{x}), \ldots, A_{k-1}(\bar{x})$. Analogous?y, each $T \in T$ is a member of one of the coalitions $A_{1}(\bar{y}), \ldots, A_{l-1}(\bar{y})$. Together with the fact that $A_{i}(\bar{x})=A_{i}(\bar{y})$ for $i<m$, this implies that $e(T, \bar{x})=e(T, \bar{y})$ for all $T \in T$.

Since $1_{S}$ can be written as a linear combination of vectors $1_{T}$ for $T \in T$, it follows that also $e(S, \bar{x})=e(S, \bar{y})$. The same reasoning as above can now 
be used to prove that $A_{m}(\bar{x})=A_{m}(\bar{y})$. Since $k$ and $l$ were both chosen maximal, it follows that $k=l$. Now it follows immediately that $\theta(\bar{x})=\theta(\bar{y})$, since otherwise we would have either $\theta(\bar{x}) \succ \theta(\bar{y})$ or $\phi(\bar{y}) \succ \phi(\bar{x})$. Hence, $\bar{x}=\bar{y}$.

The above theorem is also valid when the term nucleolus is replaced by prenucleolus. We may also replace the term nucleolus by anti-nucleolus and reverse the inequality signs.

Corollary 2.16 Let $(N, v)$, be a game and let $B \subseteq 2^{N}$ contain all essential coalitions of $v$. If Core $(v) \neq \emptyset$, then $\mathcal{N}(v)=\mathcal{N}(B, v)$.

Proof: For $x \in \operatorname{Core}(v)$ all excesses are non-positive, while for $x \notin \operatorname{Core}(v)$ at least one excess is strictly positive. It follows that $\mathcal{N}(B, v) \in \operatorname{Core}(v)$ and $\mathcal{N}(B \cup\{S\}, v) \in \operatorname{Core}(v)$ for any coalition $S$ outside $B$.

Suppose $S \notin B$. Then $S$ is not essential, and there exists a partition $\tau \subseteq B$ of $S$ such that $v(S) \leq \sum_{T \in T} v(T)$. Let $\bar{x}=\mathcal{N}(B, v)$ and let $\bar{y}=$ $\mathcal{N}(B \cup\{S\}, v)$.

We have

$$
e(S, x)=v(S)-x(S) \leq \sum_{T \in T}(v(T)-x(T))=\sum_{T \in T} e(T, x)
$$

for all $x \in \mathbb{R}^{N}$. Since $\bar{x}, \bar{y} \in \operatorname{Core}(v)$, it follows that all excesses at $\bar{x}$ and $\bar{y}$ are non-positive. Hence,

$$
e(S, \bar{x}) \leq e(T, \bar{x}) \text { for all } T \in T,
$$

and

$$
e(S, \bar{y}) \leq e(T, \bar{y}) \text { for all } T \in T .
$$

Now apply theorem 2.15 to prove that $\mathcal{N}(B, v)=\mathcal{N}(B \cup\{S\}, v)$. Repeated application of theorem 2.15 for all coalitions outside $B$ gives us the desired result.

Also in this corollary the term nucleolus can be replaced by prenucleolus, since the nucleolus and the prenucleolus coincide when Core $(v) \neq 0$. If we define a coalition to be anti-essential by reversing the inequality signs in the definition of an essential coalition, then we may also replace essential by anti-essential, core by anti-core, and nucleolus by anti-nuclecilus in the corollary. 


\subsection{The TD-property and the B-prenucleolus}

The algorithm to compute the B-prenucleolus of a game works particularly well when the collection $B$ has the TD-property. In this case the linear programs that arise have a special structure, which makes it possible to solve them efficiently. The linear programs have the following structure.

$$
\begin{array}{lll}
\mathcal{Q}(v, B, A): & \text { Minimize } & \\
\text { subject to } & x(S)=v(S) & S \in A \subseteq B \\
& x(S)+t \geq v(S) & S \in B \backslash A .
\end{array}
$$

In this formulation the collection $A$ is chosen as follows. If $\operatorname{Core}(B, v) \neq 0$, then there exists a collection $A$ such that $\{N\} \subseteq A \subseteq B$ and such that $x(S)=v(S)$ for all $x \in \operatorname{Core}(B, v)$ and all $S \in A$. We then choose a collection $A$ with these properties. If $\operatorname{Core}(B, v)=\emptyset$, we choose $A=\{N\}$.

Assume that $B$ has the TD-property. For each $t \in \mathbb{R}$ we define the game $v_{t}$ by

$$
v_{t}(S)= \begin{cases}v(S) & \text { if } S \in A \\ v(S)-t & \text { if } S \in B \backslash A .\end{cases}
$$

Notice that finding the optimal value of problem $\mathcal{Q}(v, B, A)$ is equivalent to finding the minimal $t$ for which the game $v_{t}$ has a non-empty $B$-restricted core, and according to theorem 2.12 the latter is equivalent to finding the minimal $t$ for which the grand coalition $N$ is $B$-stable in $v_{t}$. In the following example we suggest an approach to solve problem $Q(v, B, A)$.

Example : Let $N=\{1,2,3,4,5\}$ and let $G=(N, E)$ be a path graph, where $E=\{\{i, i+1\} \mid i \in\{1,2,3,4\}\}$. Furthermore, let $B \subseteq 2^{N}$ be the collection of coalitions which are connected in the path $G$. Hence, $B$ has the TD-property. Define the game $(N, v)$ by $v(N)=10, v(23)=v(345)=3$, $v(123)=4, v(234)=v(1234)=5$, and $v(2345)=8$. For the remaining coalitions the value is 0 . Take $A=\{N\}$.

We want to find the minimal $t$ for which $N$ is $B$-stable in the game $v_{t}$. Let us denote this minimal $t$ by $\bar{t}$. We suggest the following procedure to find $\bar{t}$.

Choose $t_{0}$ such that the game $v_{t_{0}}$ is not stable. In general, $t_{0}=-(n+2) M$ will do the trick, where $M=\max \{|v(S)| \mid S \subseteq N\}$ and $n$ is the number of players in the game (this will be shown later). So choose $t_{0}=-70$. Now we use the procedure in the proof of theorem 2.10 to determine a maximal $B$-partition in $v_{t_{0}}$. This partition is the partition of $N$ into singletons, which 
has value 350. Let us denote this partition by $S^{0}$. Clearly, $t_{0}=-70$ is a lower bound for $\bar{t}$.

Now we set $t_{1}$ such that the partition $S^{0}$ just does not violate the stability of $N$ in the game $v_{t_{1}}$. So we set $t_{1}=-2$, since $\sum_{i \in N} v_{-2}(i)=10=v_{-2}(N)$. For $t<t_{1}=-2$, the game $v_{t}$ is certainly not stable, since then the partition $S^{0}$ violates the stability conditions. So also $t_{\mathbf{i}}=-2$ is a lower bound for $\bar{t}$.

We do not have the garantuee that $N$ is stable in the game $v_{t_{1}}$. Again, we use the procedure in the proof of theorem 2.10 to determine a maximal $B$-partition in $v_{t_{1}}$. This gives us the partition $S^{\mathbf{1}}=\{1,2345\}$, which has value 12 in the game $v_{t_{1}}$. We conclude that $v_{t_{1}}$ is not stable, so we are not finished yet. Now, set $t_{2}$ such that the partition $S^{1}$ just does not violate the stability of $N$ in the game $v_{t_{2}}$. So we set $t_{2}=-1$. Again, $t_{2}=-1$ is a lower bound for $\bar{t}$. But this time $N$ is stable in the game $v_{t_{2}}$, so $t_{2}$ is also an upper bound for $\bar{t}$. We conclude that $\bar{t}=t_{2}=-1$, and we have solved problem $\mathcal{Q}(v, B, A)$. See also the table below, where we have summarized our findings in this example.

\begin{tabular}{|l||l|l|l|l|l|}
\hline & & & $t_{0}=-70$ & $t_{1}=-2$ & $t_{2}=-1$ \\
\hline$S$ & & $v$ & $v_{t_{0}}$ & $v_{t_{1}}$ & $v_{t_{2}}$ \\
\hline 1 & & 0 & 70 & 2 & 1 \\
2 & 0 & 70 & 2 & 1 \\
3 & 0 & 70 & 2 & 1 \\
4 & 0 & 70 & 2 & 1 \\
5 & 0 & 70 & 2 & 1 \\
12 & 0 & 70 & 2 & 1 \\
23 & 3 & 73 & 5 & 4 \\
34 & 0 & 70 & 2 & 1 \\
4.5 & 0 & 70 & 2 & 1 \\
123 & 4 & 74 & 6 & 5 \\
2.34 & 5 & 75 & 7 & 6 \\
345 & 3 & 73 & 5 & 4 \\
1234 & 5 & 75 & 7 & 6 \\
2345 & 8 & 78 & 10 & 9 \\
$N$ & fixed & 10 & 10 & 10 & 10 \\
\hline & & & $S^{0}=\{1,2,3,4,5\}$ & $S^{1}=\{1,2345\}$ & stable \\
\hline
\end{tabular}

Let us formalize the ideas in the example. Let $M=\max \{|v(S)| \mid S \subseteq N\}$ and let $n$ be the number of players in the game $v$. Consider the following algorithm to solve problem $\mathcal{Q}(v, B, A)$. 
$t_{0}:=-(n+2) M ; i:=0$

determine a maximal B-partition $S^{0} \subseteq B$ of $N$ in the game $v_{t_{0}}$; while $v_{t_{i}}$ is not stable

do

$$
t_{i+1}:=\left(\sum_{S \in S^{i}} v(S)-v(N)\right) /\left|S^{i} \backslash A\right|
$$

determine a maximal B-partition $S^{i+1} \subseteq \mathcal{E}$ of $N$

in the game $v_{t_{i+1}}$;

$$
i:=i+1
$$

od.

Let us show that the algorithm terminates, and correctly solves problem $\mathcal{Q}(v, B, A)$. We may assume that $A$ does not contain all 1-person coalitions, since otherwise the game $v$ has exactly one core element, which must be the (pre)nucleolus of $v$. In this case is is unnecessary to solve problem $\mathcal{Q}(v, B, A)$. So assume that $A$ contains at least one 1-person coalition. Then $N$ is not stable in the game $v_{t_{0}}$, since $\sum_{i \in N} v_{t_{0}}(i) \geq(n+2) M-n M=2 M>v(N)$. This shows that $t_{0}$ is a lower bound for $\bar{t}$.

While the game $v_{i}$ is not stable, a maximal B-partition of $v_{t_{i}}$ always contains at least one element outside $A$. When $A=\{N\}$, this is trivial. When $A \neq\{N\}$, we know that $\operatorname{Core}(B, v) \neq \emptyset$. It follows that for a partition $S \subseteq A$ of $N$ we have

$$
\sum_{S \in S} v_{t_{i}}(S)=\sum_{S \in S} v(S) \leq v(N)=v_{t_{i}}(N) .
$$

Since $v_{t_{i}}$ is not stable, we know that the maximal partition $S^{i+1}$ must satisfy $\sum_{S \in S^{i+1}} v_{t_{i}}(S)>v_{t_{i}}(N)$. This partition must then contain at least one element outside $A$. Hence, $\left|S^{i} \backslash A\right|>0$, and division by zero will not occur in the algorithm. Furthermore, each $t_{i}$ in the algorithm is a lower bound for the optimal value of problem $\mathcal{Q}(v, B, A)$. For $t_{0}$ we have already shown this. For $i>0$ we have

$$
t_{i}\left|S^{i-1} \backslash A\right|=\sum_{S \in S^{i-1}} v(S)-v(N) .
$$

Using the definition of $v_{t_{i}}$ we find

$$
\sum_{S \in S^{i-1}} v_{t_{i}}(S)=v(N) .
$$

So the value $t_{i}$ is chosen such that the partition $S^{i-1}$, which violated the stability of $v_{t_{i-1}}$ just does not violate the stability of $v_{t_{i}}$. It follows that 
$v_{t}$ is not stable when $t<t_{i}$, implying that $t_{i}$ is a lower bound for problem $\mathcal{Q}(v, B, A)$. If the algorithm ends, say with $i=k$, then $v_{t_{k}}$ is stable, and consequently $t_{k}$ is an upper bound for problem $\mathcal{Q}(v, 8, A)$. We conclude that if the algorithm terminates, then it terminates with the optimal value of $\mathcal{Q}(v, B, A)$. Furthermore, $\bar{A}:=S^{k-1} \backslash A \neq \emptyset$ and

$$
\sum_{S \in S^{k-1}} v_{t_{k}}(S)=v(N)
$$

so that $x(S)+t_{k}=v(S)$ for all optimal solutions $\left(x, t_{k}\right)$ of $\mathcal{Q}(v, B, A)$ and $S \in \bar{A}$.

It remains to prove that the algorithm indeed terminates. First we shall prove that the sequence $t_{0}, t_{1}, \ldots$ is increasing. To show that $t_{i+1}>t_{i}$ observe that

$$
v(N)=\sum_{S \in S^{i}} v_{t_{i+1}}(S)=\sum_{S \in S^{i}} v(S)-t_{i+1}\left|S^{i} \backslash A\right|,
$$

and

$$
v(N)<\sum_{S \in S^{i}} v_{t_{i}}(S)=\sum_{S \in S^{i}} v(S)-t_{i}\left|S^{i} \backslash A\right| .
$$

This can only be the case if $t_{i+1}>t_{i}$.

Now we shall prove that $\left|S^{i} \backslash A\right|$ is strictly decreasing. Assume that the algorithm has not terminated yet at stage $i+1$, so that $N$ is not $B$-stable in the game $v_{t_{i+1}}$. Furthermore, suppose $\left|S^{i+1} \backslash A\right| \geq\left|S^{i} \backslash A\right|$. Then we have

$$
\begin{aligned}
& \sum_{S \in S^{i+1}} v_{t_{i+1}}(S)=\sum_{S \in S^{i+1}} v(S)-t_{i+1}\left|S^{i+1} \backslash A\right| \\
& =\sum_{S \in S^{i+1}} v_{t_{i}}(S)+\left(t_{i}-t_{i+1}\right)\left|S^{i+1} \backslash A\right| \\
& \leq \sum_{S \in S^{i}} v_{t_{i}}(S)+\left(t_{i}-t_{i+1}\right)\left|S^{i} \backslash A\right| \\
& =\sum_{S \in S^{i}} v_{t_{i+1}}(S)=v(N) \text {, }
\end{aligned}
$$

contradicting the fact that $N$ is not $B$-stable in the game $v_{t_{i+1}}$ and that $S^{i+1}$ is a maximal $B$-partition of $N$ in this game. The algorithm starts with $\left|S^{0} \backslash A\right| \leq n$ and in each step the value $\left|S^{i} \backslash A\right|$ decreases at least by one. It follows that the algorithm terminates within $n$ steps.

Let us continue our example and see how we can find the $B$-prenucleolus by solving a sequence of problems of the type $Q(v, B, A)$.

Example: Let $N, B, A=\{N\}$ and $v$ be as in the previous example. We set $A^{0}=A$ and $v^{0}=v$. Earlier, we have solved problem $Q\left(v^{0}, B, A^{0}\right)$ successfully and found that the optimal value is $\bar{t}=-1$. Let us denote 
the game $v_{t}^{0}$ by $v^{1}$. According to theorem 2.14 we have that $P \mathcal{N}\left(B, v^{0}\right)=$ $P \mathcal{N}\left(B, v^{1}\right)$, so we are left with the computation of the B-prenucleolus of $v^{1}$.

Observe that $v^{1}(1)+v^{1}(2345)=1+9=10=v^{1}(N)$. Hence, $x(1)=v^{1}(1)$ and $x(2345)=v^{1}(2345)$ for all $x \in \operatorname{Core}\left(v^{1}\right)$. This is no coincidence, it is the consequence of equation (2.3) applied on the game $v_{t_{2}}$ and the partition $S^{1}$ in the previous example. We update $A^{1}=A^{0} \cup\{1,2345\}$ and solve problem $\mathcal{Q}\left(v^{1}, B, A^{1}\right)$ in the same way we solved $\mathcal{Q}\left(v^{0}, B, A^{0}\right)$. In the table below we see how repeatedly a problem of the type $\mathcal{Q}\left(v^{i}, B, A^{i}\right)$ is solved. The process ends when $A^{3}$ contains all singletons: the game $v^{2}$ has only one core-element, and this must then be the B-prenucleolus of $v$.

\begin{tabular}{|l||l|l|l|l|l|}
\hline & & & $t_{0}=-70$ & $t_{1}=-5 / 4$ & $t_{2}=-1$ \\
\hline$S$ & & $v^{1}$ & $v_{t_{0}}$ & $v_{t_{1}}^{1}$ & $v_{t_{2}}^{1}$ \\
\hline 1 & fixed & 1 & 1 & 1 & 1 \\
2 & & 1 & 71 & $9 / 4$ & 2 \\
3 & & 1 & 71 & $9 / 4$ & 2 \\
4 & & 1 & 71 & $9 / 4$ & 2 \\
5 & & 1 & 71 & $9 / 4$ & 2 \\
12 & & 1 & 71 & $9 / 4$ & 2 \\
23 & & 4 & 74 & $21 / 4$ & 5 \\
34 & & 1 & 71 & $9 / 4$ & 2 \\
45 & & 1 & 71 & $9 / 4$ & 2 \\
123 & & 5 & 75 & $25 / 4$ & 6 \\
234 & & 6 & 76 & $21 / 4$ & 7 \\
345 & & 4 & 74 & $29 / 4$ & 5 \\
1234 & 6 & 76 & 9 & 7 \\
2345 & fixed & 9 & 9 & 10 & 9 \\
$N$ & fixed & 10 & 10 & $S^{1}=\{1,23,4,5\}$ & stable \\
\hline & & & $S^{0}=\{1,2,3,4,5\}$ & \\
\hline
\end{tabular}

Update $A^{2}:=A^{1} \cup\{1,23,4,5\}$ and $v^{2}:=v_{-1}^{1}$. 


\begin{tabular}{|l||l|l|l|l|}
\hline & & & $t_{0}=-70$ & $t_{1}=-1 / 2$ \\
\hline$S$ & & $v^{2}$ & $v_{t_{0}}^{2}$ & $v_{t_{1}}^{2}$ \\
\hline 1 & fixed & 1 & 1 & 1 \\
2 & & 2 & 72 & $5 / 2$ \\
3 & & 2 & 72 & $5 / 2$ \\
4 & fixed & 2 & 2 & 2 \\
5 & fixed & 2 & 2 & 2 \\
12 & & 2 & 72 & $5 / 2$ \\
23 & fixed & 5 & 5 & 5 \\
34 & & 2 & 72 & $5 / 2$ \\
45 & & 2 & 72 & $5 / 2$ \\
123 & & 6 & 76 & $13 / 2$ \\
234 & & 7 & 77 & $15 / 2$ \\
345 & & 5 & 75 & $11 / 2$ \\
1234 & & 7 & 77 & $15 / 2$ \\
2345 & fixed & 9 & 9 & 9 \\
$N$ & fixed & 10 & 10 & 10 \\
\hline & & & $S^{0}=\{1,2,3,4,5\}$ & stable \\
\hline
\end{tabular}

Update $A^{3}:=A^{2} \cup\{1,2,3,4,5\}$ and $v^{3}:=v_{-1 / 2}^{2}$. The collection $A^{3}$ contains all singletons. Furthermore, we know that $x(S)=v^{3}(S)$ for all $S \in A^{3}$ and all $x \in \operatorname{Core}\left(B, v^{3}\right)$. Hence, $v^{3}$ has exactly one element in the $B$-restricted core: $x=\left(v^{3}(1), v^{3}(2), v^{3}(3), v^{3}(4), v^{3}(5)\right)=(1,5 / 2,5 / 2,2,2)$. This must then be the B-prenucleolus of $v^{3}$ and of $v=v^{0}$.

In each step of the algorithm it is necessary to determine a maximal $B$-partition of $N$ in the game $v_{t_{i}}$. We have seen in section 2.3 that it takes $O(n|B|)$ elementary operations to determine such a partition. Consequently, the algorithm to solve problem $Q(v, B, A)$ has complexity $O\left(n^{2}|B|\right)$. Problem $\mathcal{Q}(v, B, A)$ has to be solved $O(|B|)$ times, in order to compute the $B$-prenucleolus. This yields an $O\left(n^{2}|B|^{2}\right)$ algorithm to compute the $B$-prenucleolus of a game with the TD-property.

We mentioned in section 2.3 that the collection of essential coalitions has the TD-property for sequencing games, for the dual of minimum cost spanning tree games, and for the dual of routing games. In case the core is non-empty, the nucleolus coincides with the prenucleolus, and according to corollary 2.16 the prenucleolus coincides with the $\mathcal{E}(v)$-prenucleolus. This means that the results of this section can be applied directly to sequencing games and to the dual of minimum cost spanning tree games to compute the 
nucleolus of these games. For the dual of a routing game it should be checked whether the core is non-empty. Since sequencing games and the dual of a routing game only have $O\left(n^{2}\right)$ essential coalitions, where $n$ is the number of players, this yields an $O\left(n^{6}\right)$ algorithm to compute the nucleolus of these games in case of a non-empty core. However, for these games it is possible to compute a maximal partition of $N$ in $O\left(n^{2}\right)$ elementary operations instead of $O\left(n^{3}\right)$ (see chapter 3 , the remark after theorem 3.2). It follows that the nucleolus can be computed in $O\left(n^{5}\right)$ elementary operations.

\subsection{Location games}

In this section we want to point out the relationships between the fractional partitioning problem that we have discussed in section 2.3 and another well-known optimization problem in literature, called the fractional covering problem. This relationship is expressed in the similarity of theorem $\mathbf{2 . 1 0}$ of section 2.3 and theorem 2.21 of this section. In terms of a game $v$ and a collection $B$ of formable coalitions, the fractional covering problem can be formulated as $\operatorname{FCP}(B, v)$

$$
\begin{array}{lll}
\min & \sum_{B \in B} \lambda_{B} v(B) & \\
\text { subject to } & \sum_{B: i \in B} \lambda_{B} \geq 1 & \text { for all } i \in N \\
& \lambda_{B} \geq 0 & \text { for all } B \in B
\end{array}
$$

The dual problem is

$$
\begin{array}{ll}
\max & x(N) \\
\text { subject to } & x(B) \leq v(B) \quad \text { for all } B \in B \\
& x \geq 0 .
\end{array}
$$

Since the dual problem searches for a non-negative element in the 8 -restricted anti-core of the game $v$, we shall refer to this problem as the positive anticore problem or $\operatorname{PACP}(B, v)$. The problems $\operatorname{FCP}(B, v)$ and $\operatorname{PACP}(B, v)$ are discussed in Hoffman, Kolen and Sakarovitch[26]. Hoffman et al. give conditions such that $\operatorname{FCP}(B, v)$ and $\operatorname{PACP}(B, v)$ can be solved efficiently. We shall discuss these conditions.

Index the elements of $B$ and define the matrix $A^{B}$ by

$$
A_{i j}^{81}= \begin{cases}1 & \text { if } i \in B_{j} \\ 0 & \text { otherwise. }\end{cases}
$$


The matrix $A^{B}$ is called totally balanced if it does not contain a square submatrix of size at least three, whose row and column sums are equal to two, and which has no two identical columns. Hoffman et al. show that $\operatorname{FCP}(B, v)$ and $\operatorname{PACP}(B, v)$ are easily solved when the matrix $A^{B}$ is totally balanced. By permuting rows and columns, a totally balanced matrix can be transformed into a matrix with a much simpler structure. A $(0,1)$-matrix is called a row inclusion matrix if it does not contain the submatrix $F=\left(\begin{array}{ll}1 & 1 \\ 1 & 0\end{array}\right)$.

\section{Lemma 2.17 Row inclusion matrices are totally balanced.}

Proof: Let $A$ be a row inclusion matrix. Suppose $A$ is not totally balanced. Then it contains a square submatrix $B$ of size at least 3 , whose row and column sums are all equal to 2 , and which has no two identical columns. Then there exist $i, j, k, l$ with $i<j$ and $l<k$ such that $B_{i l}=B_{i k}=B_{j l}=1$. Then $B_{j k}=0$, since otherwise $B$ contains two identical columns. Hence, $A$ contains the submatrix $F$, a contradiction.

In the book of Nemhauser and Wolsey[39] the following lemma is proved. We do not include a proof of this lemma, since its proof is rather lengthy.

Lemma 2.18 Any totally balanced matrix can be transformed into a row inclusion matrix by permuting its rows and columns.

As a consequence of lemma 2.18 , totally balancedness of the matrix $A^{B}$ can be expressed as follows in terms of the collection $B$. A player $i \in N$ is said to have the inclusion property with respect to $B$ if for all $A, B \in B$ with $i \in A \cap B$ we have that $A \subseteq B$ or $B \subseteq A$. The collection $B$ is said to have the total inclusion property (TI-property) if, possibly after renumbering the players, player $k$ has the inclusion property with respect to the collection $B_{k}=\{B \cap\{k, \ldots, n\} \mid B \in B\}$.

Lemma 2.19 The matrix $A^{B}$ is totally balanced if and only if the collection $B$ has the TI-property.

Proof: Suppose $A^{B}$ is totally balanced. According to lemma 2.18 we may assume that the elements of $B$ and the players in $N$ are indexed such that $A^{8}$ does not contain the submatrix $F$. Choose $i \in N$ and $B_{k}, B_{l} \in B$ such that $i \in B_{k} \cap B_{l}$. Assume that $l<k$. We shall prove that $B_{l} \cap\{i, \ldots, n\} \subseteq$ 
$B_{k} \cap\{i, \ldots, n\}$. Suppose this is not true. Then we choose $j>i$ such that $j \in B_{l}$ and $j \notin B_{k}$. Define

$$
A=\left(\begin{array}{ll}
A_{i l}^{B} & A_{i l}^{B} \\
A_{i k}^{B} & A_{j k}^{B}
\end{array}\right) .
$$

Observe that $A=F$, a contradiction. We conclude that $B$ has the TIproperty.

Now suppose that $B$ has the TI-property. For any two subsets $S, T \subseteq N$ $(S \neq T)$, let us say that $S$ is lexicographically greater than $T$ or $S \succ T$, if $\max (S \cup T) \backslash(S \cap T) \in S$. Now, arrange the elements of $B$ such that $B_{k}>B_{k}$ if $k>l$, and construct the matrix $A^{B}$ with respect to this indexation (the index of players is increasing from top to bottom, and the index of coalitions is increasing from left to right). We shall prove that $A^{B}$ is a row inclusion matrix. Suppose this is not true. Then $A^{8}$ contains $F$ as a submatrix. Equivalently, we can choose $i, j \in N$ with $j>i$ and we can choose $B_{l}, B_{k} \in B$ with $k>l$, such that $i \in B_{l}, i \in B_{k}, j \in B_{l}$ and $j \notin B_{k}$. Furthermore, since $B_{k} \succ B_{l}$, it follows that $h=\max \left(B_{l} \cup B_{k}\right) \backslash\left(B_{l} \cap B_{k}\right) \in B_{k}$. Observe that $h>j$.

Now,

$$
B_{l} \cap\{i, \ldots, n\} \nsubseteq B_{k} \cap\{i, \ldots, n\},
$$

since $j \in B_{l}$ and $j \notin B_{k}$. On the other hand

$$
B_{k} \cap\{i, \ldots, n\} \notin B_{l} \cap\{i, \ldots, n\},
$$

since $h \in B_{k}$ and $h \notin B_{l}$. This contradicts the TI-property. We conclude that $A^{B}$ does not contain $F$ as a submatrix, and hence $A^{B}$ is a row inclusion matrix.

Next we shall prove that problems $\operatorname{FCP}(B, v)$ and $\operatorname{PACP}(B, v)$ are easily solved when $B$ has the TI-property and $v \geq 0$. Define the vector $z^{B}$ defined recursively by

$$
z_{k}^{B}=\min \left\{v(B)-z^{B}(B \cap\{1, \ldots, k-1\}) \mid k \in B \in B\right\} .
$$

Lemma 2.20 The vector $z^{B}$ is a feasible solution of $P A C P(B, v)$ if $v \geq 0$.

Proof: Let $B \in B$ and let $k$ be the largest index in $B$. By definition we have $z_{k}^{B} \leq v(B)-z^{B}(B \cap\{1, \ldots, k-1\})=v(B)-z^{B}(B \backslash\{k\})$. It follows that $z^{\hat{B}}(B) \leq v(B)$. 
It remains to prove that $z^{B} \geq 0$. Let $k \in N$. Choose $\bar{B} \in B$ with $k \in \bar{B}$ such that

$$
z_{k}^{B}=v(\bar{B})-z^{B}(\bar{B} \cap\{1, \ldots, k-1\}) .
$$

If $\bar{B} \cap\{1, \ldots, k-1\}=\emptyset$ then it follows immediately that $z_{k}^{B}=v(\bar{B}) \geq 0$. Otherwise, choose $l$ maximal in $\bar{B} \cap\{1, \ldots, k-1\}$. It follows immediately from the definition of $z_{l}^{\beta}$ that $z_{l}^{\beta} \leq v(\bar{B})-z_{l}^{B}(\bar{B} \cap\{1, \ldots, l-1\})$, and hence, $z^{B}(\bar{B} \cap\{1, \ldots, l\}) \leq v(\bar{B})$. Now it follows that

$$
z_{k}^{B}=v(\bar{B})-z^{B}(\bar{B} \cap\{1, \ldots, k-1\})=v(\bar{B})-z^{B}(\bar{B} \cap\{1, \ldots, l\}) \geq 0 .
$$

Theorem 2.21 The vector $z^{B}$ defined by (2.4) is an optimal solution of $\operatorname{PACP}(B, v)$ if $B$ has the TI-property.

Proof: According to the duality theorem of linear programming it suffices to construct a cover for $N$ with value $z^{B}(N)$. Let $B_{k}$ denote a minimizing coalition in

$$
\min \left\{v(B)-z^{B}(B \cap\{1, \ldots, k-1\}) \mid k \in B \in B\right\},
$$

and among these minimizing coalitions, choose the one which is lexicographically maximal. Furthermore, define the collections $C_{k}(k \in N)$, recursively as follows.

$$
\begin{array}{ll}
C_{n}=\left\{B_{n}\right\}, & \\
C_{k}= \begin{cases}C_{k+1} & \text { if } k \text { is contained in one of the elements of } C_{k+1} \\
C_{k+1} \cup\left\{B_{k}\right\} & \text { otherwise. }\end{cases}
\end{array}
$$

$C_{1}$ is trivially a cover for $N$. It remains to prove that its value equals $z^{B}(N)$.

Suppose $j \in N$ is covered twice by $C_{1}$, i.e. there exist $k$ and $l(k \neq l)$, such that $B_{k}, B_{l} \in C_{1}$ and $j \in B_{k} \cap B_{l}$. Assume that $l<k$. Observe that $j \neq l$, since $l \notin B_{k}$. We shall prove that $j>l$. Suppose this not true, i.e. $j<l$. Because of the TI-property, we must have $B_{k} \cap\{j, \ldots, n\} \subseteq B_{l} \cap\{j, \ldots, n\}$ or $B_{l} \cap\{j, \ldots, n\} \subseteq B_{k} \cap\{j, \ldots, n\}$. Since $l \notin B_{k}$ the latter is not possible, so it follows that $B_{k} \cap\{j, \ldots, n\} \subseteq B_{l} \cap\{j, \ldots, n\}$, and that $B_{l}>B_{k}$. Furthermore, $z^{B}\left(B_{l}\right) \geq z^{B}\left(B_{l} \cap\{1, \ldots, k\}\right) \geq z^{B}\left(B_{l} \cap\{1, \ldots, l\}\right)=v\left(B_{l}\right)$. We also have $z^{B}\left(B_{l}\right) \leq v\left(B_{l}\right)$. Combining this we find $z^{B}\left(B_{l} \cap\{1, \ldots, k\}\right)=$ $v\left(B_{l}\right)$. We see that $B_{l}$ is also a minimizing coalition in

$$
\min \left\{v(B)-z^{B}(B \cap\{1, \ldots, k-1\}) \mid k \in B \in B\right\},
$$


contradicting the fact that $B_{k}$ was chosen lexicographically maximal. We conclude that the assumption $j<l$ is false, and hence $j>l$.

Since $j>l$, it follows that

$$
z^{B}\left(B_{l}\right) \geq z^{B}\left(B_{l} \cap\{1, \ldots, j\}\right) \geq z^{B}\left(B_{l} \cap\{1, \ldots, l\}\right)=v\left(B_{l}\right) .
$$

Moreover, $z^{8}\left(B_{l}\right) \leq v\left(B_{l}\right)$ and it follows that $z^{8}\left(B_{l} \cap\{1, \ldots, j\}\right)=v\left(B_{l}\right)$. Now we find that

$$
z_{j}^{B}=v\left(B_{l}\right)-z^{B}\left(B_{l} \cap\{1, \ldots, j-1\}\right) \leq v\left(B_{l}\right)-z^{B}\left(B_{l} \cap\{1, \ldots, l\}\right)=0,
$$

and we conclude $z_{j}^{\beta}=0$. So for all $j \in N$ which appear more than once in the cover $C_{1}$ we have $z_{j}^{B}=0$. Hence,

$$
\sum_{B \in C_{1}} z^{8}(B)=z^{B}(N)
$$

For $B \in C_{1}$ we have $z^{B}(B)=v(B)$, so it follows that

$$
\sum_{B \in C_{1}} v(B)=z^{8}(N)
$$

Observe that the proof of theorem 2.21 is very similar to that of theorem 2.10. First, an integral feasible solution for $\operatorname{FCP}(B, v)$ is constructed, much in the same way as the partition was constructed for $\operatorname{FPP}(B, v)$. Then it is shown that the value of this solution equals the value of $z^{B}$.

Totally balanced matrices arise in the formulation of some location problems. Let $G=(V, E)$ be an undirected, connected graph and $d: E \rightarrow \mathbb{R}^{+}$a weight function on the edges of $G$. For each pair of vertices $v, w \in V$ the distance $d(v, w)$ is defined as a minimum weight path between $v$ and $w$, where the weight of a path is defined as the sum of the weights of the edges that lie on this path. A subset $N \subseteq V$ is interpreted as a set of customers who need to have have some kind of service center, for instance a supermarket, in their neighborhood. Each customer $i \in N$ requires that at least one service center should be located at a distance not exceeding some non-negative number $M_{i}$. Furthermore, let $Q \subseteq V$ denote the set of possible locations for a service center and let $k_{q}$ denote the cost of placing a service center in $q \in Q$. The problem is to find a set of locations for service centers, such that the total cost is minimized and such that the requirements of all customers are satisfied. It is assumed that at least one feasible set of locations exists. 
The location problem can be formulated as a set covering problem as follows. For each $q \in Q$ let $B(q) \subseteq N$ denote the set of customers who are satisfied when a service center is placed at location $q$. Furthermore, let $B=\{B(q) \mid q \in Q\}$ and for each $B \in B$ define $c(B)=\min \left\{k_{q} \mid B(q)=B\right\}$. The location problem is now equivalent to solving problem $\operatorname{FCP}(B, c)$. The following theorem is due to Hoffman et al.[26].

Theorem 2.22 If the underlying graph $G$ of the location problem considered above is a tree, then the matrix $A^{B}$ in the formulation as a set covering problem is totally balanced.

It follows that the location problem can be solved efficiently if the underlying graph is a tree.

In a natural way the location problem gives rise to a location game, where the cost $c(S)$ is taken to be the worth of an optimal solution which satisfies the requirements of coalition $S$. The following result is due to Tamir[56].

Theorem 2.23 Consider the setting above, where a graph $G=(V, E)$, a weight function $d^{t}: E \rightarrow \mathbb{R}^{+}$, a set of players $N \subseteq V$, a set of possible locations for service centers $Q \subseteq V$ and a cost function $k: Q \rightarrow \mathbb{R}^{+}$determines a location game $(N, c)$. If the graph $G$ is a tree, then $c$ has a non-empty anti-core.

Proof: The matrix $A^{B}$ is totally balanced. Hence, the vector $z^{B}$ defined by $(2.4)$ is an optimal solution for $\operatorname{PACP}(B, c)$.

From the feasibility of $z^{B}$ it follows that $z^{B}(B) \leq c(B)$ for all $B \in B$. Let $S \subseteq N$. The worth $c(S)$ is defined as the value of an optimal cover for $S$. Let $S \subseteq B$ be such an optimal cover. We then have $c(S)=\sum_{B \in S} c(B)$. Using the non-negativity of $z^{B}$ it follows that

$$
z^{B}(S) \leq \sum_{B \in S} z^{B}(B) \leq \sum_{B \in S} c(B)=c(S) .
$$

The worth $c(N)$ of the grand coalition is by definition equal to the optimal cover for $N$. Since the optimal values of $\operatorname{PACP}(B, c)$ and $\operatorname{FCP}(B, c)$ coincide, it follows that $z^{B}(N)=c(N)$. We conclude that $z^{B}$ is an element of ACore (c). 


\section{Chapter 3}

\section{Traveling salesman games}

Section 3.2 of this chapter is based on Derks and Kuipers[14]. Section 3.3 is based on Kuipers[28].

\subsection{Introduction}

Consider the following problem. A repairman has to visit a number of customers. He starts in his home city, visits each customer, and returns home. The total cost of his trip has to be paid by the customers. The problem is to find a fair allocation of the costs among the customers. This type of problem was first investigated by Fishburn and Pollack[17]. In Potters, Curiel and Tijs[46] the problem was tackled with techniques from cooperative game theory. Their approach was to associate a cooperative game with the allocation problem. Techniques from game theory can then be applied to find reasonable outcomes. However, several games can be associated with the allocation problem. Potters et al. consider two types of games.

The first class of games Potters et al. considered are traveling salesman games. Let $N=\{1, \ldots, n\}$ denote the set of customers and let 0 denote the repairman. Furthermore, let $G$ be a directed graph with node set $N \cup\{0\}$, such that for each ordered pair $i, j \in N \cup\{0\}$ there is a directed path from $i$ to $j$. Furthermore, a non-negative weight is assigned to each arc in the graph. A tour for coalition $S$ is a roundtrip along the arcs in the graph of the repairman, who starts in 0, visits each customer in $S$ at least once, and returns home. The length of the tour is defined as the sum of the arc weights on this tour. The cost of a coalition $S$ is defined as the length of a minimum length tour. This cost is denoted by $c(S)$. Potters et al. give 
sufficient conditions on the weights of the arcs in the graph, such that a traveling salesman game (TSG) has a non-empty anti-core. A 3-person TSG always has a non-empty anti-core. However, the anti-core of a TSG can be empty. Potters et al. gave an example of a 4-person game with an empty anti-core in which the arc weights of the graph are not symmetric, i.e. the cost to travel from $i$ to $j$ differs from the cost to travel from $j$ to $i$. Even if these weights are symmetric a TSG can have an empty anti-core. Tamir[57] gave an example of a 6-person symmetric TSG for which the anti-core is empty. Using a result of Fonlupt and Naddef[18] he also showed that a symmetric TSG always has a non-empty anti-core when there are at most 4 players. The question remained whether a 5-person symmetric TSG always has a non-empty anti-core. The answer is yes, and the proof is given in section 3.3 .

The second class of games Potters et al. considered was the following. Here, the game is defined on a complete directed graph. The cost of the grand coalition $N$ is the sum of the arc weights in an arbitrarily chosen tour traversed by the repairman, who starts at node 0 , visits each customer exactly once, and returns home. Such a tour can be represented by a permutation $\tau=\left(\tau_{1}, \ldots, \tau_{n}\right)$ of the elements of $N$, where $\tau_{i}$ is the customer who is visited in $i$-th place. This cost is denoted by $c_{r}(N)$. Each subset $S \subseteq N$ of customers can also hire the repairman to serve its members only. For such a subset $S$ a tour is constructed from $\tau$ by visiting the customers in $S$ in the same order as they were visited in the tour $\tau$, and by skipping the other customers. The cost for coalition $S$ is the sum of the arc weights on this tour. The cost is denoted by $c_{\tau}(S)$. A game defined in this way is called a fixed-route traveling salesman game or a routing game.

Potters et al. proved that a routing game has a non-empty anti-core if the weights in the graph $G$ satisfy the triangle inequalities and if the tour $r$ which is chosen for the grand coalition is a tour of minimum length. In section 3.2 we give an alternative proof of this result. This proof has the advantage that it provides an efficient algorithm for constructing an element in the anti-core. In that section we also prove that a routing game has a nonempty anti-core if the tour $\tau$ for the grand coalition is an adapted nearest neighbour tour.

Keeping the order of the players fixed makes a routing game mathematically tractable, while results for a traveling salesman game are much harder to obtain. However, the routing game model seems not very satisfactory from an economic point of view. It is likely that a coalition will object to a proposed sharing of the costs, when this coalition can do better on its 
own by rearranging the order in which its members are visited, or by visiting some players or the home city more than once. If in such a situation the repairman is indeed capable and willing to deviate from the fixed order tour, then the TSG model should be prefered above the routing game model. However, one can think of situations in which the repairman cannot deviate from the fixed order tour. This could be the case when the repairman made an appointment with each individual player to meet him at a specified time. Rearranging the order of the players means that some appointments have to be canceled. This is not in the interest of the players if they have tight schedules with other activities as well. Another good reason for considering the routing game model is that there exist interesting instances where the characteristic functions of the routing game and the traveling salesman game coincide.

Suppose the repairman repairs yachts. He lives at the coast of an island and his customers all live at the coast too. Furthermore, assume that travel costs are proportional to the Euclidian distances. In this case it is wellknown that an optimal tour is cross-free, i.e. the repairman should not come across a point in the Euclidian plane more than once. This is still true if we restrict ourselves to some connected area in the Euclidean plane (e.g. an island). If all the cities lie on the boundary of this area, as in the case of our repairman, there are only two tours that are cross-free: the tour which visits the cities in the order as we meet thern when we go clockwise along the boundary of the area, and the tour which goes anti-clockwise along the boundary of the island. When travel costs are symmetric, both tours must be optimal. An optimal tour for a subset of cities can be found in the same way, by going clockwise or anti-clockwise along the boundary of the island, thereby skipping the cities that are not in the subset. This is exactly the tour that the repairman would follow in a routing game. Hence, the routing game and the traveling salesman game coincide, and the nice mathematical results for routing games can be applied to the traveling salesman game as well.

\subsection{Routing games}

\subsubsection{Introduction}

In this section we consider the class of routing games into more detail. As a first result we show that the anti-core of a routing game may be empty. Consider the graph in figure 3.1, let $\tau=(1,2,3)$ and $\sigma=(1,3,2)$. 


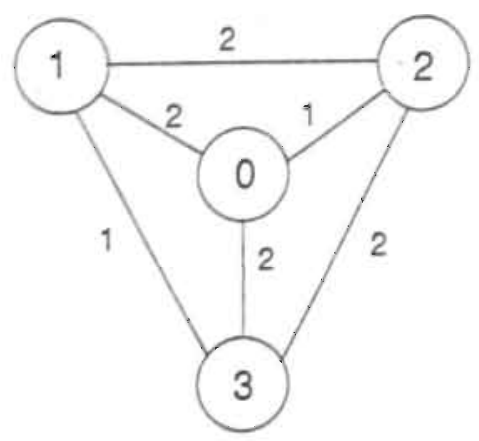

Figure 3.1

The corresponding characteristic functions $c_{\tau}$ and $c_{\sigma}$ are given in the table below.

\begin{tabular}{|c||r|r|r|r|r|r|r|}
\hline$S$ & 1 & 2 & 3 & 12 & 13 & 23 & 123 \\
\hline$c_{\tau}$ & 4 & 2 & 4 & 5 & 5 & 5 & 8 \\
\hline$c_{\sigma}$ & 4 & 2 & 4 & 5 & 5 & 5 & 6 \\
\hline
\end{tabular}

Suppose that $x \in \mathbb{R}^{3}$ is a vector in the anti-core of $c_{r}$. Then $x(12) \leq$ $c_{\tau}(12)=5, x(13) \leq c_{\tau}(13)=5$ and $x(23) \leq c_{\tau}(23)=5$. Thus, $x(123)=$ $\frac{1}{2} x(12)+\frac{1}{2} x(13)+\frac{1}{2} x(23) \leq 7 \frac{1}{2}<8=c_{\tau}(123)$, a contradiction. This shows that the anti-core of $c_{\tau}$ is empty. Clearly, the emptiness of the anti-core depends on the choice of the tour along $N \cup\{0\}$, since $(2,2,2)$ is an element of the anti-core of $c_{\sigma}$.

In section 3.2.2 we address the following problem. Let $G$ be a graph with node set $N \cup\{0\}$ and let $\tau$ be a tour along this node set. Is the anti-core of the associated routing game empty or not? And is it possible to compute an element in the anti-core efficiently if it is non-empty? We show that the anticore of a routing game can be described by at most $\frac{1}{2} n(n+1)$ restrictions, and we provide an $O\left(n^{2}\right)$ algorithm which computes an allocation in the anti-core if it is non-empty. Furthermore, we show in this section that the anti-core of a routing game is non-empty under the mild condition $c(N) \leq c(S)+c(N \backslash S)$ for all $S \subseteq N$. A game with this property is called $N$-subadditive. In section 3.2 .3 we provide some procedures to construct a tour $\tau$ which will guarantee that the corresponding routing game is $\mathrm{N}$-subadditive. 


\subsubsection{The anti-core of a routing game}

Let $(N, v)$ be a game. Recall that the dual of a game is defined by $v^{*}(S)=$ $v(N)-v(N \backslash S)$ for all $S \subseteq N$ and that Core $\left(v^{*}\right)=\operatorname{ACore}(v)$.

In the following we shall see that the number of essential coalitions in the dual $c^{*}$ of a routing game $c$ with $n$ players is at most $\frac{1}{2} n(n+1)$. Hence, $\operatorname{Core}\left(c^{*}\right)=\operatorname{ACore}(c)$ can be described by only $\frac{1}{2} n(n+1)$ restrictions.

Let $G$ be the complete directed graph with node set $V=N \cup\{0\}$ and let $d: V \times V \rightarrow \mathbb{R}^{+}$be a weight function on the arcs of $G$. Furthermore, let $\tau$ be a tour that visits each node in $V$ exactly once and let $c$ be the routing game associated with $\tau$. Without loss of generality assume that on this tour the players are visited in the order $1,2, \ldots, n$. Let $i, j \in N$ with $i \leq j$. We denote the coalition $\{k \in N \mid i \leq k \leq j\}$ by $[i, j]$.

Lemma 3.1 Let $(N, c)$ be a routing game. Then, possibly after reindexing the players, all essential coalitions of the dual $c^{*}$ are of the form $[i, j]$.

Proof: Let us first express the dual value of a coalition $[i, j]$ in terms of arc weights in the graph. We have

$$
c(N \backslash[i, j])=\sum_{h=1}^{i-1} d(h-1, h)+d(i-1, j+1)+\sum_{h=j+2}^{n+1} d(h-1, h)
$$

and

$$
c(N)=\sum_{h=1}^{n+1} d(h-1, h),
$$

where arithmetic is done modulo $n$. Hence,

$$
c^{*}([i, j])=c(N)-c(N \backslash[i, j])=\sum_{h=i}^{j+1} d(h-1, h)-d(i-1, j+1) .
$$

In figure 3.2 the arcs that have a positive sign in this expression are indicated with ' + ', and the arc that has a negative sign is indicated with '-'.

Now, let $S \subseteq N . S$ can be partitioned into coalitions of the form $\left[i_{k}, j_{k}\right]$, $k=1, \ldots, K$, where the coalitions $\left[i_{k}, j_{k}\right]$ are taken maximal w.r.t. inclusion. Furthermore, define $j_{0}=-1$ and $i_{K+1}=n+2$. Then

$$
\begin{aligned}
c^{*}(S)= & c(N)-c(N \backslash S) \\
= & \sum_{h=1}^{n+1} d(h-1, h)- \\
& \left.\sum_{k=1}^{K+1} \sum_{h=j_{k-1}+2}^{i_{k}-1} d(h-1, h)-\sum_{k=1}^{K} d\left(i_{k}-1, j_{k}+1\right)\right) \\
= & \sum_{k=1}^{K}\left(\sum_{h=i_{k}}^{j_{k}+1} d(h-1, h)-d\left(i_{k}-1, j_{k}+1\right)\right) \\
= & \sum_{k=1}^{K} c^{*}\left(\left[i_{k}, \hat{j}_{k}\right]\right) .
\end{aligned}
$$


We conclude that in the dual of a routing game only coalitions of the form $[i, j]$ can be essential.

In figure 3.3 we have pictured a coalition $S$ which consists of two components, $S_{1}$ and $S_{2}$. To compute the dual value of $S$ we have to add the weights of the arcs that have a positive sign in this picture, and subtract the arcs with a negative sign. This is precisely the sum of the dual values of $S_{1}$ and $S_{2}$.

Observe that there are only $\frac{1}{2} n(n+1)$ coalitions of type $[i, j]$. Hence, the anti-core of a routing game can be described by at most $\frac{1}{2} n(n+1)$ linear restrictions. In Curiel, Pederzoli and Tijs[11] it is proved that also sequencing games possess this property.

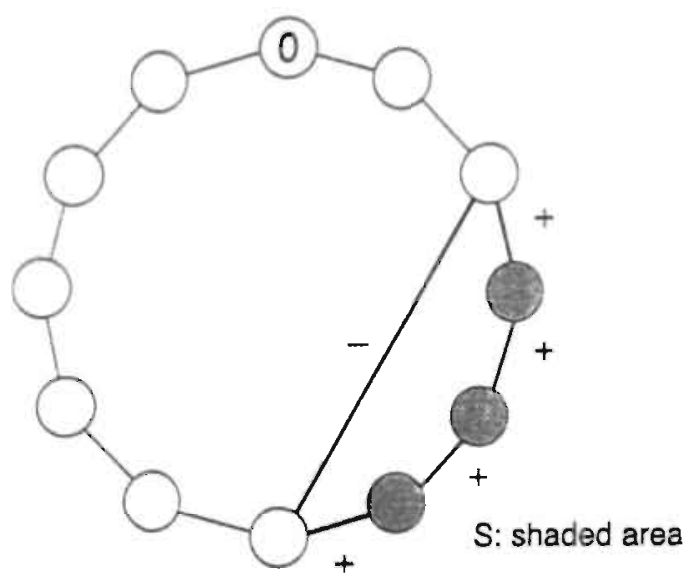

Figure 3.2

Let $P$ be the graph with vertex set $N=\{1, \ldots, n\}$ and edge set $E=$ $\{\{i, i+1\} \mid i=1,2, \ldots, n-1\}$. Then the subgraph on a coalition of the type $[i, j] \subseteq N$ is a connected graph. Hence, all essential coalitions of the dual of a routing game are connected in the path graph $P$, and it follows that the collection of essential coalitions has the TD-property. Hence, we can apply the findings of chapter 2 to deduce the following result.

Theorem 3.2 Let $(N, c)$ be a routing game. Define $x=\left(x_{1}, \ldots, x_{n}\right)$ recur- 


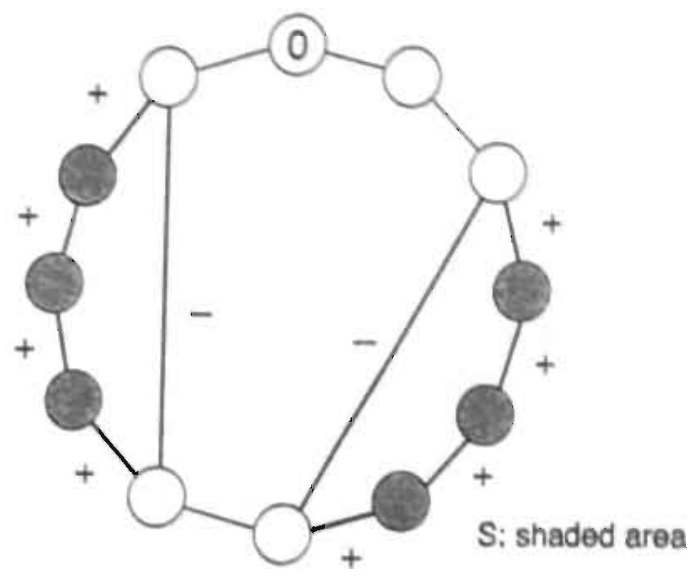

Figure 3.3

sively by

$$
x_{k}=\max _{j=1}^{k}\left(c^{*}([j, k])-x([j, k-1])\right) .
$$

Then $x \in \operatorname{Core}\left(c^{*}\right)(=A \operatorname{Core}(c))$ if and only if the grand coalition is stable in $c^{*}$.

Proof: Observe that the vector $x$ defined by (3.1) coincides with the vector $y^{B}$ defined by $(2.2)$ when we take $B=\{[i, j] \mid 1 \leq i \leq j \leq n\}$. According to theorem 2.12 we have $y^{B} \in \operatorname{Core}\left(B, c^{*}\right)$ if and only if $N$ is $B$-stable. Since $B$ contains all essential coalitions, the restricted core coincides with the core and $B$-stability is equivalent to stability. It follows that $x=y^{B} \in \operatorname{Core}\left(c^{*}\right)=\operatorname{ACore}(c)$ if and only if $N$ is stable in the game $c^{*}$.

The vector $x$ defined by (3.1) can be computed in $O\left(n^{2}\right)$ elementary operations. Thus, it is possible to verify in $O\left(n^{2}\right)$ time whether a routing game has an empty anti-core or not. At the same time an element in the anti-core is computed in case it is non-empty.

Theorem 3.3 A routing game has a non-empty anti-core if and only if the game is $N$-subadditive.

Proof: $N$-subadditivity of a game is trivially a necessary condition for the non-emptiness of the anti-core. It remains to prove that for routing games 
this is also a sufficient condition. According to theorem 3.2 it suffices to prove that $N$-subadditivity of a routing game $c$ implies the stability of $N$ in the dual game $c^{*}$. A maximal partition of $N$ in the game $c^{*}$ can be found among partitions into essential coalitions. Let $T=\left(\left[i_{k}, j_{k}\right]\right)_{k=1}^{K}$ be such a maximal partition of $N$ with $1=i_{1}<i_{2}<\ldots<i_{K}$ and $j_{k}=i_{k+1}-1$ for $k=1, \ldots, K-1$, and $j_{K}=n$. Define $S_{\text {even }}$ as the union of coalitions with even index in this partition, and $S_{\text {odd }}$ as the union of coalitions with odd index. We have seen earlier that the dual value of a coalition equals the sum of the values of its maximal components. Thus, $c^{*}\left(S_{\text {even }}\right)=\sum_{k \text { even }} c^{*}\left(\left[i_{k}, j_{k}\right]\right)$ and $c^{*}\left(S_{\text {odd }}\right)=\sum_{k \text { odd }} c^{*}\left(\left[i_{k}, j_{k}\right]\right)$. Using the $N$-subadditivity of $c$ we find

$$
\begin{aligned}
\sum_{k=1}^{K} c^{*}\left(\left[i_{\boldsymbol{k}}, j_{k}\right]\right) & =c^{*}\left(S_{\text {even }}\right)+c^{*}\left(S_{\text {odd }}\right)= \\
& =2 c(N)-\left(c\left(S_{\text {even }}\right)+c\left(S_{\text {odd }}\right)\right) \leq \\
& \leq c(N)=c^{*}(N) .
\end{aligned}
$$

Thus, $N$ is stable in $c^{*}$.

\subsubsection{Routing games with a non-empty anti-core}

In the previous subsection we did not put any restrictions on the tour for the grand coalition in a routing game and as we have seen in subsection 3.2.1, non-emptiness of the anti-core may very well depend on the choice of this tour. In this section we adress the problem of constructing tours, which guarantee the non-emptiness of the anti-core. This is not always possible. Consider the symmetric case in which the weight of an edge $\{i, 0\}$ is 0 for all $i \in N$ and in which the weight of an edge $\{i, j\}$ is 1 for all $i, j \in N$. In this case the game has an empty anti-core, no matter which tour is chosen for the grand coalition.

The weight function $d$ is said to satisfy the triangle inequalities if

$$
d(i, j) \leq d(i, k)+d(k, j)
$$

for all $i, j, k \in N \cup\{0\}$. In the example above the weight function does not satisfy the triangle inequalities. It turns out that it is always possible to construct a tour $r$ such that the associated routing game $c_{r}$ has a nonempty anti-core if the weight function satisfies the triangle inequalities. In fact, even the weaker condition

$$
d(i, j) \leq d(i, 0)+d(0, j)
$$


for all $i, j \in N$ is sufficient to guarantee the existence of such a tour. We shall say that $d$ satisfies weak triangle inequalities if it satisfies (3.2).

Theorems 3.4 and 3.5 provide two methods for constructing tours which induce a routing game with a non-empty anti-core. Theorem 3.4 , which states that a minimum length tour has the desired property, was proved earlier by Potters, Curiel and Tijs. They provided two different proofs. In [46] they define an associated game $\bar{c}$ with a routing game $c$. The associated game satisfies $\bar{c}(S) \leq c(S)$ for all $S \subseteq N$ and $\bar{c}(N)=c(N)$. Furthermore, $\bar{c}$ is a linear production game in the sense of Owen[43]. Since these games have a non-empty anti-core, it follows that $\mathrm{ACore}(c) \supseteq \operatorname{ACore}(\bar{c}) \neq \emptyset$. Another proof by Potters et al. is given in [47]. Here they give a direct proof that the balancedness conditions of Shapley-Bondareva hold, when the repairman travels a tour of minimum length. We provide an alternative proof which makes use of theorem 3.3.

Theorem 3.4 Let $G$ be the complete directed graph with node set $N \cup\{0\}$ and let $d$ be a non-negative weight function on the arcs of $G$ which satisfies weak triangle inequalities. Let $(N, c)$ be the routing game associated with a minimum length tour $\tau$ along $N \cup\{0\}$. Then $c$ has a non-empty anti-core.

Proof: According to theorem 3.3 it is sufficient to prove that $c$ satisfies the $N$-subadditivity condition $c(N) \leq c(S)+c(N \backslash S)$ for all $S \subseteq N$. Let $S \subseteq N, \tau_{1}$ the tour that results from $\tau$ by skipping the players in $N \backslash S$, and $\tau_{2}$ the tour that results from $\tau$ by skipping the players in $S$. Construct the tour $\sigma$ as follows. Start in 0 and follow the tour $\tau_{1}$ up to the last player, say $i$ in $S$. Then, instead of returning to 0 , jump to the first player, say $j$, in $\tau_{2}$. Finally, follow $\tau_{2}$ until the home city 0 is reached. Using the triangle inequality $d_{i j} \leq d_{i 0}+d_{0 j}$, it follows that the length of $\sigma$ is at most the sum of the lengths of $\tau_{1}$ and $\tau_{2}$. Since $\tau$ is a minimum length tour, it also follows that the length of $\tau$ is at most the sum of the lengths of $\tau_{1}$ and $\tau_{2}$, i.e. $c(N) \leq c(S)+c(N \backslash S)$.

Constructing a minimum length tour for the grand coalition is not the only way of ensuring the non-emptiness of the anti-core. An adapted nearest neighbour tour also possesses this property.

Two games $v$ and $w$ are called strategically equivalent if $\alpha>0$ and $\beta \in \mathbb{R}^{n}$ exist such that $v(S)=\alpha w(S)+\beta(S)$ for all $S \subseteq N$. Clearly, the core (anticore) of $v$ is empty if and only if the core (anti-core) of $w$ is empty. Now, consider the following adaptation of the weight function $d$. Choose $i \in N$ 
and $\epsilon>0$ and raise the weights of all outgoing arcs from $i$ with the amount $\epsilon$. Denote the adapted weight function by $\tilde{d}$. The weight function $\tilde{d}$ satisfies the weak triangle inequalities if $d$ does. Let $\tau$ be a tour along $N \cup\{0\}$, and let $c$ and $\tilde{c}$ be the routing games associated with $r$ and the weight functions $d$ and $\tilde{d}$ respectively. We have

$$
\tilde{c}(S)= \begin{cases}c(S) & \text { if } i \notin S \\ c(S)+\epsilon & \text { if } i \in S .\end{cases}
$$

Thus, $c$ and $\tilde{c}$ are strategically equivalent. The same is true if we raise all ingoing arcs from $i$. Now, let $m$ be the maximum weight of the $\operatorname{arcs}(0, i)$ and $(i, 0)(i \in N)$. First, raise all outgoing arcs from player 1 such that the arc $(1,0)$ gets weight $m$. Proceed with player 2,3 , etc. until all arcs $(i, 0)$ have weight $m$. Analogously, raise the ingoing arcs from all players such that all arcs $(0, i)$ get weight $m$. Finally, divide all arc weights by $m$. Let us denote the adapted weight function by $\bar{d}$. Let $c$ be a routing game corresponding to some tour $r$ and to the original weight function $d$, and let $\bar{c}$ be the routing game corresponding to the same tour $\tau$ and to the adapted weight function $\bar{d}$. Then $c$ and $\bar{c}$ are strategically equivalent. The weight function $\bar{d}$ satisfies the weak triangle inequalities if $d$ does. Observe that for $\bar{d}$ the weak triangle inequalities are equivalent to the property $\bar{d}(i, j) \leq 2$ for all $i, j \in N$.

The following theorem states that a routing game has a non-empty anticore if $\tau$ is a nearest neighbour tour with respect to the adapted weight function $\bar{d}$.

Theorem 3.5 Let $G$ be the complete directed graph with node set $N \cup\{0\}$ and let $d$ be a non-negative weight function on the arcs of $G$ which satisfies $d(0, i)=d(i, 0)=1$ for all $i \in N$, and $d(i, j) \leq 2$ for all $i, j \in N$. Let $\tau$ be a nearest neighbour tour along $N \cup\{0\}$ starting with the home city 0 and let $c$ be the routing game associated with $\tau$. Then $c$ has a non-empty anti-core.

Proof: Without loss of generality we assume that $(1,2, \ldots, n)$ is a nearest neighbour tour. Then we have $d(i, i+1) \leq d(i, j)$ for all $i \in N$ and all $j \in N$ with $j \geq i+1$. According to theorem 3.3 it is sufficient to prove the $N$-subadditivity of $c$. Let $S \subseteq N, \tau_{1}$ the tour that results from $\tau$ by skipping the players in $N \backslash S$ and $\tau_{2}$ the tour that results from skipping the players in $S$. If $i \in S$ we denote by $f(i)$ the follower of $i$ in the tour $\tau_{1}$. If $i \in N \backslash S$ then $f(i)$ is the follower of $i$ in the tour $\tau_{2}$. Clearly, we have either $f(i) \geq i+1$ or $f(i)=0$. There are two players $i \in N$ with $f(i)=0$, namely the players 
which are visited last in $\tau_{1}$ and $\tau_{2}$. One of them must be player $n$. Let us denote the other player by $k$. Then we have

$$
\begin{aligned}
c(S)+c(N \backslash S) & =2+\sum_{i \in N} d(i, f(i)) \geq \\
& \geq 4+\sum_{i \neq k, n} d(i, i+1) \geq \\
& \geq 2+d(k, k+1)+\sum_{i \neq k, n} d(i, i+1)= \\
& =2+\sum_{i \neq n} d(i, i+1)=c(N) .
\end{aligned}
$$

Both the minimum tour and the nearest neighbour tour have a serious drawback. Finding a minimum tour is a well-known $N P$-hard problem, so that for a large number of players the problem becomes intractable. On the other hand, the ratio between the length of a nearest neighbour tour and an optimal tour can be arbitrarily bad (see e.g. Papadimitriou and Steiglitz[45]. This may be unacceptable to the players, who are naturally interested in a low cost for the grand coalition.

Suppose that some approximation algorithm, no matter which one, is applied for finding a sub-optimal tour $\tau$ for the grand coalition. If a good approximation algorithm is used, then the cost for the grand coalition will not be too high. However, we do not have the guarantee then that the associated routing game has a non-empty anti-core. We shall describe an $O\left(n^{2}\right)$ procedure, which takes $\tau$ as its input, checks whether the routing game associated with $\tau$ has an element in the anti-core, and computes a tour $\sigma$ of strictly lesser length than $\tau$ if this is not the case.

Let $\left(N, c_{\tau}\right)$ be a routing game associated with a tour $\tau$ for the grand coalition. First, determine a maximal partition $S_{1}, \ldots, S_{k}$ of $N$ in the game $c_{r}^{*}$. Such a partition can be found using the algorithm of theorem 2.10. The complexity of this algorithm applied on a routing game is $O\left(n^{2}\right)$.

The elements of the partition are all of the type $[i, j]$. Without loss of generality we assume that the elements of the partition are indexed in such a manner that the last player in $S_{i}$ and the first player in $S_{i+1}$ are consecutive players for $i=1,2, \ldots, k-1$. Now, let $S=\cup_{k \text { odd }} S_{k}$. According to lemma 3.1 we have

$$
c_{r}^{*}(S)=\sum_{k_{\text {odd }}} c_{r}^{*}\left(S_{k}\right)
$$

and

$$
c_{r}^{*}(N \backslash S)=\sum_{k \text { even }} c_{\tau}^{*}\left(S_{k}\right)
$$


Suppose that $\operatorname{Core}\left(c_{\tau}\right)=\emptyset$, and thus $N$ is not stable in the game $c_{r}^{*}$. Then

$$
c_{\tilde{\tau}}^{*}(N)<\sum_{i=1}^{\boldsymbol{k}} c_{\tau}^{*}\left(S_{\mathbf{i}}\right)=c_{\tau}^{*}(S)+c_{\tau}^{*}(N \backslash S),
$$

and hence

$$
c_{\tau}(S)+c_{\tau}(N \backslash S)=2 c_{\tau}(N)-c_{\tau}^{*}(S)-c_{\tau}^{*}(N \backslash S)<c_{\tau}(N) .
$$

Then construct the tour $\sigma$ as follows. Start in the home city 0 and visit the players in $S$ as prescribed by the tour $\tau$. From the last player in $S$, jump to the first player in $N \backslash S$. Then visit the players in $N \backslash S$ as prescribed by $r$. Finally, return to 0 . Using the triangle inequalities it follows that $c_{\sigma}(N) \leq c_{r}(S)+c_{r}(N \backslash S)<c_{r}(N)$.

Repeating the above procedure a number of times, eventually produces a tour for which the associated routing game has a non-empty anti-core. In the worst case it reaches a minimum tour after at most $n$ ! steps. We were not able to give better bounds on the number of steps needed before termination. We leave it as an open problem whether the number of steps is polynomially bounded by the number of players in the game.

\subsection{The 5-person TSG}

In this section we discuss the symmetric version of the traveling salesman garne. Therefore, the game will be defined on an undirected graph and we shall omit the word symmetric from now on. The traveling salesman problem defined on a graph $G$ may be formulated as an integer programming problem. In their paper Fonlupt and Naddef[18] consider a relaxation of the integer problem in which the integrality constraints and some other constraints are left out. They provide sufficient conditions on the graph $G$ such that the value of the relaxed problem equals the value of the original problem.

An edge contraction is the process of replacing two adjacent vertices, say $i$ and $j$, by a single vertex. As a result of this the edge $\{i, j\}$ disappears. Other edges that had $i$ or $j$ as an endpoint will now have the new vertex as an endpoint.

The conditions of Fonlupt and Naddef that ensure that the relaxed traveling salesman problem equals the value of the original problem require that $G$ contains no minor which is isomorphic to one of the following three graphs. Here, a minor of $G$ is a graph that results from $G$ by a sequence of edge deletions and edge contractions. 


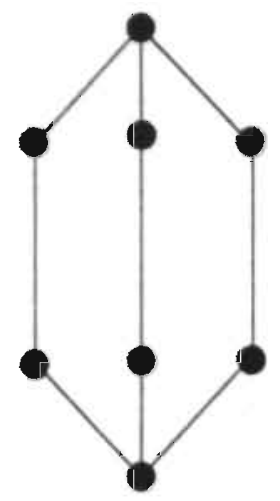

Figure 3.4

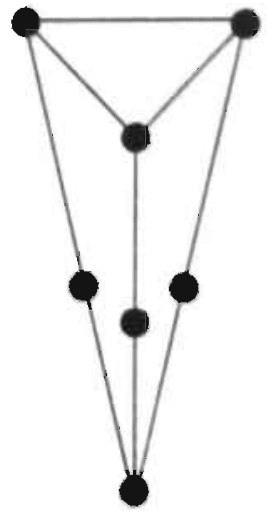

Figure 3.5

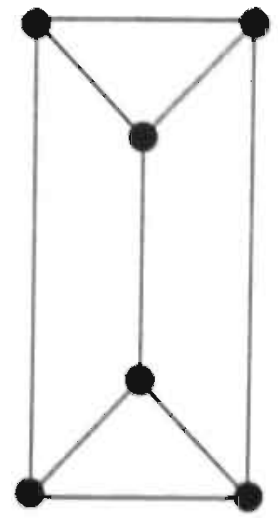

Figure 3.6

Tamir observed that as a direct consequence of this, a TSG has a nonempty anti-core if the graph $G$ contains no minor which is isomorphic to one of these graphs. An immediate corollary is that all TSG's with 4 or less players have a non-empty anti-core. Since the graph of a 5-person TSG has only six nodes, it cannot contain a minor isomorphic to one of the graphs in figure 3.4 and 3.5. Thus, a 5-person TSG can only have an empty anti-core if its graph $G$ contains a minor isomorphic to the graph in figure 3.6. Using this observation one easily proves the following two lemma's.

Lemma 3.6 Let $(N, c)$ te a 5-person TSG defined on $G=(N \cup\{0\}, E)$. If there exists a player $i \in N$ such that there are at most 2 edges in $E$ with $i$ as an endpoint, then $A C$ Core $(c) \neq 0$.

Proof: For each node of the graph in figure 3.6 there are exactly 3 edges with this node as an endpoint. Hence, $G$ cannot contain a minor isomorphic to the graph in figure 3.6. It follows immediately that the TSG has a nonempty anti-core. 
Lemma 3.7 Let $(N, c)$ be a 5-person $T S G$ defined on $G=(N \cup\{0\}, E)$ and suppose that the complement of $G$ contains a cycle on less than 6 nodes. Then $A$ Core $(c) \neq 0$.

Proof: The complement of the graph in figure 3.6 forms a cycle graph on six nodes. Thus, if a graph of six nodes contains a minor isomorphic to the graph in figure 3.6, then its complement is a minor of the cycle graph. Therefore, this complement cannot contain a cycle on less than six nodes. Consequently, $G$ cannot contain a minor isomorphic to the graph in figure 3.6. It follows that the TSG has a non-empty anti-core.

Let $c$ be a TSG defined on the graph $G=(N \cup\{0\}, E)$ with weight function $d$. In this section we find it convenient to denote the weight $d(i, j)$ by $d_{i j}$. Let $\tilde{G}$ be the complete graph with node set $N \cup\{0\}$ and define the weight $\tilde{d}_{i j}$ of an arbitrary edge $\{i, j\}$ in $\tilde{G}$ as the length of a shortest path in $G$ between the $i$ and $j$. It is obvious that $c$ is also the cost function of the TSG defined on the graph $\tilde{G}$ with weight function $\tilde{d}$. Furthermore, the weights $\tilde{d}$ satisfy the triangle inequalities, and therefore each coalition $S \subseteq N$ has a shortest tour that visits the players in $S$ exactly once. In the following it is assumed throughout that a TSG is defined on a complete graph and that the weight function satisfies the triangle inequalities, unless explicitly stated otherwise.

A cooperative game $(N, v)$ is called $p$-normalized if $v(\{i\})=p$ for all $i \in N$.

Lemma 3.8 Each TSG is strategically equivalent to a 2-normalized TSG.

Proof: Let $c$ be a TSG with weight function $d$. Choose $k$ arbitrarily in $N$ and $\epsilon>0$. Define the weight function $\hat{d}$ by

$$
\hat{d}_{i j}= \begin{cases}d_{i j} & \text { if } i, j \neq k \\ d_{i j}+\epsilon & \text { if } i=k \text { or } j=k\end{cases}
$$

Let $\hat{c}$ be the TSG associated with the weight function $\hat{d}$. It is easily verified that the weight function $\hat{d}$ still satisfies the triangle inequalities and that

$$
\hat{c}(S)= \begin{cases}c(S) & \text { if } k \notin S \\ c(S)+2 \epsilon & \text { if } k \in S\end{cases}
$$

So $\hat{c}$ is strategically equivalent to $c$. Now, let $m$ be the maximum weight between any player and 0 , i.e. $m=\max _{j \in N} d_{0 j}$. First, raise the weight of 
all edges $\{1, j\}$ such that $\{1,0\}$ gets weight $m$. Then raise the weight of all edges $\{2, j\}$ such that $\{2,0\}$ gets weight $m$. Proceed until all edges $\{j, 0\}$ have weight $m$. The game associated with the new weight function is a $2 m$-normalized TSG which is strategically equivalent to the original TSG $c$. Finally, divide all edge weights by $m$.

Notice that in a 2-normalized TSG $d_{i 0}=d_{0 i}=1$ for all $i \in N$ and that the weight of any edge $\{i, j\}$ with $i, j \in N$ is bounded by 2 , since the triangle inequalities imply $d_{i j} \leq d_{i 0}+d_{0 j}=2$.

Lemma 3.9 For each 2-normalized TSG $c$ there is a 2-normalized TSG $\hat{c}$ with the following properties.

i. $\hat{c}$ has a shortest tour for $N$ containing an edge of maximal weight, i.e. weight 2 .

ii. $\operatorname{ACore}(\hat{c}) \neq \emptyset \Rightarrow A \operatorname{Core}(c) \neq \emptyset$.

Proof: Let $c$ be a 2-normalized TSG with weight function $d$. If the game $c$ has a shortest tour for $N$ containing an edge of weight 2 , then define $\hat{c}=c$. Now suppose that each shortest tour for $N$ contains only edges that have weight less than 2. Let $\epsilon>0$. Define

$$
\hat{d}_{i j}= \begin{cases}d_{i j} & \text { if } i=0 \text { or } j=0 \text { or } d_{i j}=2 \\ d_{i j}+\epsilon & \text { otherwise }\end{cases}
$$

Note that for $\epsilon$ such that $\hat{d}_{i j} \leq 2$ for all $i, j \in N$, the weight function $\hat{d}$ still satisfies the triangle inequalities. Let $\hat{c}$ be the TSG associated with the weight function $\hat{d}$. All shortest tours for $N$ contain only edges of weight less than 2, so for $\epsilon$ small enough we have

$$
\begin{aligned}
& \hat{c}(N)=c(N)+(n-1) \epsilon \text { and } \\
& \hat{c}(S) \leq c(S)+(|S|-1) \epsilon \text { for all } S \subseteq N
\end{aligned}
$$

Suppose that $\hat{x}$ is an element in the anti-core of $\hat{c}$. Define $x_{i}=\hat{x}_{i}-\frac{n-1}{n} \epsilon$ for all $i \in N$. It is straightforward that $x \in \operatorname{ACore}(c)$. So, $\operatorname{ACore}(\hat{c}) \neq \emptyset \Rightarrow$ ACore $(c) \neq 0$. Choose $\epsilon$ such that one of the tours containing an edge of weight 2 becomes a shortest tour or such that the edge with largest weight among the edges with weight less than 2 gets weight 2. (Whatever happens first.) If $\hat{c}$ now has a shortest tour containing an edge of weight 2 , then stop. Otherwise repeat the process of raising edge weights, until an edge of 
weight 2 appears on as shortest tour.

We have seen earlier that in proving that all 5-person TSG's have a non-empty anti-core, it is allowed to restrict ourselves to TSG's defined on complete graphs with weight functions that satisfy the triangle inequalities. From the above two lemma's it follows that we may further restrict ourselves to 2-normalized 'TSG's that have an edge of weight 2 on a shortest tour. Without loss of generality we may assume that on this shortest tour the players $1,2,3,4$ and 5 are visited in this order. There are 4 possible locations for the edge of weight 2 on this tour, but for reasons of symmetry we only need to consider the cases $d_{12}=2$ and $d_{23}=2$. Both cases are pictured below. We shall refer to the first case as a type $I$ TSG and to the second case as a type II TSG.

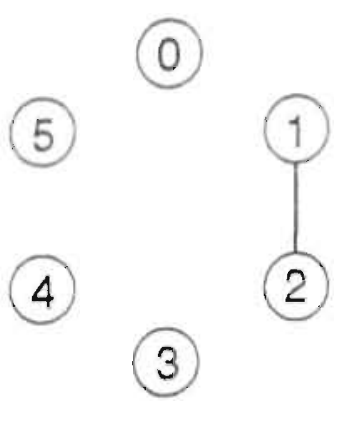

Figure 3.7 Type I TSG

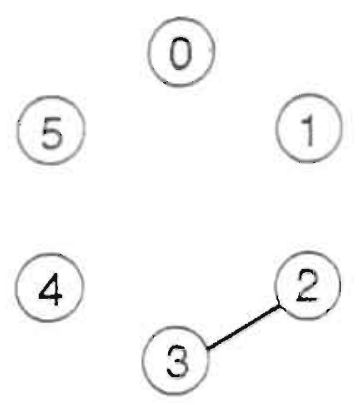

Figure 3.8 Type II TSG

Theorem 3.10 Type II TSG's have a non-empty anti-core.

Proof: We have that $[1,2,3,4,5]$ is a shortest tour and that $d_{23}=2$. The tour $[2,1,5,4,3]$ uses the edge $\{1,5\}$ instead of the edge $\{2,3\}$, but for the rest it uses edges of the same length as the tour $[1,2,3,4,5]$. We conclude that the edge $\{1,5\}$ must have weight 2 . Analogously one shows that the edges $\{1,3\}$ and $\{2,5\}$ also have weight 2 . These edges form a cycle. Remove all edges of weight 2 from the graph $G$ and denote the resulting graph by $G^{\prime}$. If a tour in the graph $G$ uses an edge $\{i, j\}$ of weight 2 , then the tour which uses the edges $\{i, 0\}$ and $\{0, j\}$ to go from $i$ to $j$ instead, has the same cost. Hence, the removal of the four edges of weight 2 does not change the characteristic 
function of the TSG. The complement of $G^{\prime}$ contains a cycle on less than 6 nodes. From lemma 3.7 it follows that the TSG has a non-empty anti-core.

\section{It remains to prove that type $I$ TSG's have a non-empty anti-core.}

Lemma 3.11 Let $c$ be a type I TSG. Then for each coalition $S$ containing both players 3 and 4 there exists a shortest tour for $S$ along the edge $\{3,4\}$.

Proof: Let $S$ be a coalition containing players 3 and 4 . The cases $|S|=2$ and $|S|=5$ are trivial. First assume that $|S|=3$, say $S=\{3,4, i\}$. There are only three possible tours for this coalition. These are $[3,4, \mathrm{i}],[4,3, \mathrm{i}]$ and $[3, i, 4]$. Since the tour $[1,3,2,4,5]$ is at least as long as $[1,2,3,4,5]$ it follows that $d_{13}+d_{24} \geq d_{12}+d_{34}=2+d_{34}$. Thus, $d_{13} \geq d_{34}$ and $d_{24} \geq d_{34}$. Analogously one shows that $d_{35} \geq d_{34}$ and $d_{14} \geq d_{34}$. So the edges $\{3, i\}$ and $\{4, i\}$ cannot both have lesser weight than $\{3,4\}$. Therefore, either $[3,4, \mathrm{i}]$ or $[4,3, \mathrm{i}]$ is at least as short as $[3, \mathrm{i}, 4]$.

Now assume that $|S|=4$. There are three coalitions of cardinality 4 that contain players 3 and 4 . These are $\{1,2,3,4\},\{1,3,4,5\}$ and $\{2,3,4,5\}$. It is obvious that $[2,3,4,5]$ is a shortest tour for coalition $\{2,3,4,5\}$.

Let $S=\{1,2,3,4\}$. If a tour for $S$ uses the edge $\{1,2\}$ then it is possible to construct another tour, which replaces $\{1,2\}$ by some other edge, but for the rest it uses the edges of the same length as the original tour. For instance, $[1,2,3,4]$ can be replaced by $[2,3,4,1]$. This tour is at least as short as the original tour. Therefore, in searching a shortest tour for $S$ we may restrict. ourselves to tours that do not make use of the edge $\{1,2\}$. Furthermore, if a shortest tour for $S$ ends with player 4 , then it is possible to construct. a shortest tour for $N$ by visiting the players in $S$ as prescribed by this tour and visit player 5 directly after player 4. Therefore, any tour for $S$ ending (or starting) with player 4 must be as least as long as $[1,2,3,4]$ and this tour is at least as long as $[1,4,3,2]$. We conclude that a shortest tour for $S$ can be found among tours that do not make use of the edge $\{1,2\}$ and do not start or end with player 4 . There are only four such tours. These are $\pi_{1}=[1,4,3,2]$, $\tau_{2}=[1,3,4,2], \tau_{3}=[1,4,2,3]$ and $\tau_{4}=[2,4,1,3]$. It was shown earlier that $d_{13}+d_{24} \geq 2+d_{34}$. From this it follows that $d_{13}+d_{24} \geq d_{23}+d_{34}$. Therefore $\tau_{4}$ cannot be shorter than $\tau_{1}$. It also follows that $d_{24} \geq d_{34}$, so $\tau_{3}$ is not shorter than $\tau_{1}$. We have proved that either $\tau_{1}$ or $\tau_{2}$ is shortest for $S$. Both tours use the edge $\{3,4\}$.

It remains to prove that a shortest tour for $S=\{1,3,4,5\}$ uses the edge $\{3,4\}$. Observe that the tour $[1,5,4,3,2]$ is at least as short as $[1,2,3,4,5]$ 
and therefore the edge $\{1,5\}$ must have weight 2. After renumbering the players: $\pi(1)=1, \pi(2)=5, \pi(3)=4, \pi(4)=3$ and $\pi(5)=2$, it is clear that this case is similar to the case $S=\{1,2,3,4\}$.

Lemma 3.12 Let c be a type I TSG. Then there exists a type I TSG $\hat{c}$ with the following properties.

i. Besides $\{1,2\}$ and $\{1,5\}$ there is another edge of weight 2 with a shortest tour along this edge.

ii. $\operatorname{ACore}(\hat{c}) \neq \emptyset \Rightarrow A \operatorname{Core}(c) \neq \emptyset$.

Proof: Let $K$ denote the set of edges $\{\{2,3\},\{2,4\},\{2,5\},\{4,5\},\{3,5\}\}$. The edges in $K$ are drawn in the picture below.

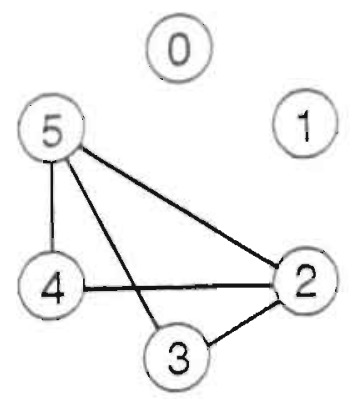

Figure 3.9

Remark that any tour for $N$ must use at least one of the edges in $K$. First suppose that there is a shortest tour for $N$, say $\tau$, that uses exactly 1 edge in $K$. Let $L$ denote the set of edges $\{\{1,3\},\{3,4\},\{1,4\}\}$ and $E$ the set of all edges between players. Since the three edges in $L$ form a cycle, any tour can use at most 2 edges in $L$ and therefore it uses at least 2 edges in $E \backslash L=K \cup\{\{1,2\},\{1,5\}\}$. Since $\tau$ uses only 1 edge in $K$, it must use one of the edges $\{1,2\}$ or $\{1,5\}$. It is easy to check that any tour starting with player 1 and then visiting player 2 or 5 must use at least 2 edges in $K$. Therefore, $\tau$ does not start or end with player 1 . Because $\tau$ contains an edge of weight 2 , also the edge between the first and the last player in this tour must have weight 2 , since else an improvement would be possible. 
Furthermore, there is a shortest tour along this edge. Since 1 is not the first or last player in $\tau$, this edge cannot be $\{1,2\}$ or $\{1,5\}$, which proves the lemma in this case.

Now suppose that all shortest tours for $N$ use at least 2 edges in $K$. Raise the weights of each edge in $K$ by $\epsilon$ if this edge has weight less than 2 . Note that the new edge weights still satisfy the triangle inequalities. Let $\hat{c}$ denote the TSG associated with the new edge weights. Since all shortest tours in the game $c$ use at least two edges in $K$, it follows that $[1,2,3,4,5]$ is still a shortest tour, provided that $\epsilon$ is small enough. Using lemma 3.11 we find

$$
\begin{array}{ll}
\hat{c}(N)=c(N)+2 \epsilon & \\
\hat{c}(S) \leq c(S)+2 \epsilon & \text { if } 2,5 \in S \\
\hat{c}(S) \leq c(S)+\epsilon & \text { if } 2 \in S, 5 \notin S \text { or } 2 \notin S, 5 \in S \\
\hat{c}(S)=c(S) & \text { if } 2,5 \notin S .
\end{array}
$$

Assume that $\hat{x} \in \operatorname{ACore}(\hat{c})$. Define $x_{j}=\hat{x}_{j}$ for $j \neq 2,5$ and $x_{j}=\hat{x}_{j}-\epsilon$ for $j=2,5$. It is staightforward that $x \in \operatorname{ACore}(c)$. Thus, ACore $(\hat{c}) \neq 0 \Rightarrow$ ACore $(c) \neq \emptyset$. We can raise the edge weights of edges in $K$ until one of the following cases occur.

i) An edge in $K$ gets weight 2 .

ii) A tour along an edge in $K$ that has weight 2 becomes a shortest tour.

iii) A tour that uses at most 1 edge in $K$ becomes a shortest tour.

If ii) or iii) occurs the proof is finished. If i) occurs we can repeat the process of raising edge lenghts until ii) or iii) occurs.

Theorem 3.13 Type I TSG's have a non-empty anti-core.

Proof: Let $c$ be a type I TSG. According to lemrna 3.12 we may assume that besides $\{1,2\}$ and $\{1,5\}$ there is another edge of weight 2 with a shortest toir along this edge. If this edge is on the tour $[1,2,3,4,5]$, then one easily shows that the game is also of type $I I$ (possibly after renumbering the players), so the game has a non-empty anti-core according to theorem 3.10. If the edge has player 1 as one of its endpoints then the anti-core is non-empty according to lemma 3.6 . If it is the edge $\{2,5\}$ the anti-core is non-empty according to lemma 3.7. So we assume that none of these situations occur. Then only the cases $d_{24}=2$ and $d_{35}=2$ remain. Both cases are easily seen 


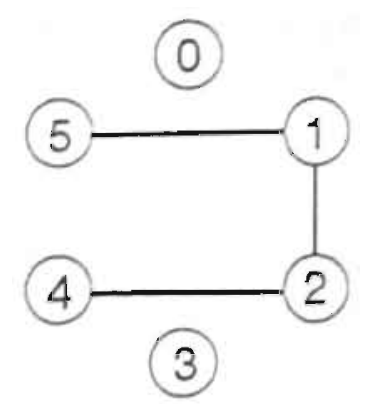

Figure 3.10

to be equivalent for reasons of symmetry. Therefore we assume that $d_{24}=2$. This situation is pictured in figure 3.10.

Let $\tau$ be a shortest tour along the edge $\{2,4\}$. If $\{2,4\}$ is the third or second edge in this tour then the game is also of type $I I$, so the garne has a non-empty anti-core according to theorem 3.10. So assume that it is the first edge in this tour. If player 4 is the first player in this tour then there must be another edge $\{4, j\}$ of weight 2 and the game has a non-empty anti-core either according to lemma 3.7 or to theorem 3.10 . So assume that player 2 is the first player in $\tau$ and 4 the second player in this tour. If 1 is not the last player in $\tau$ then there must be a third edge $\{2, j\}$ of weight 2 and the game has non-empty anti-core according to lemma 3.6. So we may assume that player 1 is the last player in this tour. There are only two tours that satisfy these conditions. These are $[2,4,3,5,1]$ and $[2,4,5,3,1]$. If $[2,4,3,5,1]$ is a shortest tour then also $[2,1,5,3,4]$ is a shortest tour, thus the game is also of type II. From theorem 3.10 it follows that the game has a non-empty anti-core. Finally suppose that $[2,4,5,3,1]$ is a shortest tour. Since the tour $[4,5,3,2,1]$ is at least as long as $[1,2,3,4,5]$ it follows that $d_{35} \geq d_{34}$. So also $[2,1,3,4,5]$ is a shortest tour and the edge $\{2,5\}$ has weight 2 . It follows both from lemma 3.6 and from lemma 3.7 that the game has a non-empty anti-core.

Corollary 3.14 All 5-person TSG's have a non-empty anti-core.

Proof: This follows directly from theorems 3.10 and 3.13 , and the fact that we may restrict ourselves to 2-normalized TSG's which have an edge of weight 2 on a shortest tour. 


\section{Chapter 4}

\section{Minimum cost spanning tree games}

Section 4.3 of this chapter is based on Kuipers[29].

\subsection{Introduction}

This chapter is based on a problem which was initially raised by Claus and Kleitman[9]. Roughly, the problem is as follows. A set of customers a.l need to be connected to a common supplier, for instance to a transmitter of cable television. This is done by establishing a set of links that connect two customers or that connect a customer to the supplier. The total cost of establishing the necessary links has to be shared by the customers. The question is how to divide this cost among the customers.

Bird[5] was the first to follow a garne theoretic approach to tackle the problem. He associated a cooperative cost game with the problem, which he called the minimum cost spanning tree (MCST) game. This way, solution concepts known in game theory can be used to solve the problem. Granot and Huberman[24] were the first to provide a correct proof that the anticore of a MCST game is never empty. This proof also provided an efficient procedure to construct an element in the anti-core.

In section 4.2 we investigate the structure of the anti-core of MCST games. As in the case of routing games it turns out to be a fruitful approach to consider the dual of a MCST game. We shall prove that the essential coalitions of the dual are connected in a forest. With this observation we shail be able to characterize a number of extreme elements of the anti-core 
of a MCST game, though certainly not all of them. In section 4.3 we restrict ourselves to MCST games for which the cost to establish a link can take only two different values, which gives rise to so-called information graph games. In this case we are able to give a nice characterization of the set of all extreme elements of the anti-core as well as the anti-nucleolus. In section 4.4 we consider a more general setting in which there are several common suppliers, each of them offering a different type of service. Each customer needs to be connected to at least one of these suppliers. We call these games minimum cost forest (MCF) games. We provide sufficient conditions such that an MCF game has a non-empty anti-core.

\subsection{The anti-core of MCST games}

Let $N=\{1, \ldots, n\}$ denote a set of customers and let 0 denote a common supplier to which the customers need to be connected. To each pair $\{i, j\}$ with $i, j \in N \cup\{0\}$ a non-negative number $d_{i j}$ is assigned, which denotes the cost of establishing a link between $i$ and $j$. The customers need to be connected to the common supplier by establishing a suitable set of links. Let us denote this set of links by $E$. The goal of connecting all customers to the common supplier is achieved if the graph with node set $N \cup\{0\}$ and edge set $E$ is a connected graph. The total cost of establishing the links in this graph is $\sum_{\{i, j\} \in E} d_{i j}$. If we assume that the customers want to be connected to the supplier at minimal cost, then the links in $E$ will form a spanning tree on $N \cup\{0\}$ of minimal cost. If a coalition $S \subseteq N$ decides to operate on its own, it is assumed that they are not allowed to establish a link which involves a customer outside $S$. Hence, the cost of coalition $S$ is the cost of a minimum cost spanning tree on the nodes $S \cup\{0\}$. Let us denote this cost by $c(S)$. A game defined in this way is called a minimum cost spanning tree game or shortly a MCST game.

Granot and Huberman[23] proved that the anti-core of a MCST game is never empty and they provide the following procedure to construct an element in the anti-core. Let $\Gamma$ denote a minimum cost spanning tree for the grand coalition. For every customer $i \in N$ let $f(i)$ denote the node which follows $i$ on the path in $\Gamma$ from $i$ to the common supplier 0 . Define

$$
x_{i}=d_{i, f(i)} \text { for all } i \in N .
$$

We shall refer to this vector as the tree vector. We include a proof of Granot and Huberman's theorem, since it is instructive to see that the fundamental idea, in this proof is also used in the proof of theorem 4.5 and theorem 4.25. 
Theorem 4.1 (Granot and Huberman) The tree vector defined by (4.1) is an element of the anti-core of the MCST game $c$.

Proof: Let $x$ be the tree vector defined by (4.1). It is trivial that $x(N)=$ $c(N)$. Let $S \subseteq N$. It remains to prove that $x(S) \leq c(S)$. In order to prove this, we construct a graph with node set $N \cup\{0\}$ in the following way. First, construct a minimum spanning tree with node set $S \cup\{0\}$. Then add the vertices in $N \backslash S$ together with the edges $\{i, f(i)\}$ for $i \in N \backslash S$. The resulting graph, which we shall denote by $G$, is connected. To see this, we choose $i \in N$ and we construct a path in $G$ from $i$ to 0 . If $i \in S$, then a path in $G$ from $i$ to 0 exists, since $G$ contains a spanning tree on $S \cup\{0\}$. If $i \in N \backslash S$, then follow the path $i, f(i), f(f(i)), \ldots$ until a node in $S \cup\{0\}$ is reached. This will surely happen, since the edges $\{i, f(i)\}$ for $i \in N \backslash S$ contain no cycle. Once a node in $S \cup\{0\}$ is reached it is trivial again that a path to 0 exists. Since we can reach 0 from every $i \in N$, we conclude that $G$ is connected. Therefore, the costs of construction are at least $c(N)$. Thus,

$$
x(N \backslash S)+c(S) \geq c(N) .
$$

Now, using that $x(N)=c(N)$, we find that

$$
x(S)=x(N)-x(N \backslash S) \leq c(S) .
$$

Notice that the tree vector is a marginal allocation vector, i.e. there exist coalitions $S_{1} \subseteq S_{2} \subseteq \ldots \subseteq S_{n}$ with the property $x\left(S_{k}\right)=c\left(S_{k}\right)$ and $\left|S_{k}\right|=k$ for all $k \in\{1, \ldots, n\}$. Hence, the tree vector is an extreme element of the anti-core.

Assume for a moment that the customers in a coalition $S$ are allowed to use links which involves customers outside $S$. Let $d(S)$ denote the cost of coalition $S$ in this model. Observe that $d(S)=\min \{c(T) \mid S \subseteq T\}$, where $c(S)$ denotes the cost of $S$ in the ordinary MCST game. Consequently ACore $(d)=\operatorname{ACore}(c) \cap \mathbb{R}_{+}^{n}$. It follows that the tree vector is not only in the anti-core of the MCST game $c$, but also in the anti-core of the game $d$. Notice that $d(S) \leq d(T)$ if $S \subseteq T$. For this reason the game $d$ is called a monotonic MCST game. From now we discuss again the ordinary MCST game model.

Granot and Huberman argue in [24] that the tree vector will often be considered unfair, since players which are connected directly to the supplier 
will have to pay their link to the supplier, while other players profit from this link without contributing to it. Therefore, it is desirable to further investigate the structure of the anti-core. In their paper Granot and $\mathrm{Hu}$ berman give procedures to generate other vectors in the anti-core. One of these procedures is called the weak demand operation. Although they do not mention it explicitly in their paper, it follows easily from their results on the weak demand operation that for each player $i \in N$ there exists an extreme element in the anti-core in which player $i$ pays his marginal contribution to the grand coalition, i.e. $c(N)-c(N \backslash\{i\})$. In this section we prove a generalization of this result. If a coalition $S \subseteq N$ is stable in the dual game $c^{*}$, then there exists an extreme element $x$ in the anti-core of $c$, which satisfies $x(S)=c(N)-c(N \backslash S)=c^{*}(S)$.

In [24] Granot and Huberman proved a theorem which they called the minimum excess coalition structure theorem. This theorem implies that all essential coalitions of the dual of a MCST game are connected in a forest with node set $N$. Their proof of the theorem contains some statements which are presented as being trivial, but which are not trivial at all in our opinion. Therefore we provide our own proof.

In the next lemma we use the following notation. Let $V$ be a finite set and let $E \subseteq V \times V$ be a collection of edges. Then for any $U \subseteq V$ we denote the set $\{\{v, w\} \in E \mid v \in U$ and $w \in U\}$ by $E_{U}$.

Lemma 4.2 Let $\Gamma=(V, E)$ be a tree and let $U \subseteq V$ be a set whose components with respect to $\Gamma$ are $U_{1}, \ldots, U_{k}$. Furthermore, let $\Gamma_{V \backslash U}=$ $\left(V \backslash U, E_{V \backslash U}\right)$ be the forest which results from $\Gamma$ when all vertices of $U$ are removed. Finally, let $F$ be a collection of edges such that $\left(V \backslash U, E_{V \backslash U} \cup F\right)$ forms a tree. Then there exists a partition $F_{1}, \ldots, F_{k}$ of $F$ such that all graphs $\left(V \backslash U_{i}, E_{V \backslash U_{i}} \cup F_{i}\right)$ are connected.

Proof: For all $f \in F$ define $I(f)$ as the set of indices $i$ for which the path between the endpoints of $f$ in the tree $\Gamma$ has a non-empty intersection with $U_{i}$. I $(f) \neq \emptyset$ for all $f \in F$, since otherwise the addition of $f$ to the forest $\Upsilon_{V \backslash U}$ would create a cycle.

Let us first consider the case that $|I(f)|=1$ for all $f \in F$, and hence $\sum_{j \in F}|I(f)|=|F|$. Then define $F_{i}=\{f \in F \mid I(f)=\{i\}\}$. Trivially, $\left(F_{i}\right)_{i=1}^{k}$ forms a partition of $F$ (possibly some of the sets in this partition are empty). It remains to prove that all graphs $\left(V \backslash U_{i}, E_{V \backslash U_{i}} \cup F_{i}\right)$ are connected. Obviously, the graph $\left(V \backslash U_{i}, E_{V \backslash U_{i}} \cup F\right)$ is connected. Choose $f \in F \backslash F_{i}$. The path between the endpoints of $f$ in $\Gamma$ does not meet the set $U_{i}$. Therefore, 
this path is also present in $\left(V \backslash U_{i}, E_{V \backslash U_{i}} \cup F\right)$, and it follows that $f$ lies on a cycle in this graph. Therefore, $f$ can be removed, while the resulting graph remains connected. Now choose another edge in $F \backslash F_{i}$. Again, the path between the endpoints of this edge in $\Gamma$ does not meet the set $U_{i}$, and the path does not use any edge we have already removed, since we do not remove edges in $\Gamma$. Now, apply the same reasoning to find that also this edge can be removed without disturbing the connectedness of the graph. This way, we can sequentially remove all edges in $F \backslash F_{i}$ and we conclude that the graph $\left(V \backslash U_{i}, E_{V \backslash U_{i}} \cup F_{i}\right)$ is connected.

Now we shall prove that the lemma also holds when $\sum_{f \in F}|I(f)|=M>$ $|F|$. Assume the lemma holds when $\sum_{f \in F}|I(f)|<M$. Since $\left.M\right\rangle|F|$, there exists an edge $\bar{f} \in F$ such that $|I(\bar{f})| \geq 2$. Removal of the edge $\bar{f}$ from the graph $\Gamma_{V \backslash U}=\left(V \backslash U, E_{V \backslash U} \cup F\right)$ causes the graph to break into two components. Let us denote the vertex sets of these components by $L$ and $R$. Let $\left(v_{0}, \ldots, v_{h}\right)$ be the path in $\Gamma$ between the endpoints of $\bar{f}=\left\{v_{0}, v_{h}\right\}$. Assume that $v_{0} \in L$ and $v_{h} \in R$. For two adjacent vertices $v_{j}$ and $v_{j+1}$ on this path it is impossible that one of them lies in $L$ and that the other lies in $R$, since otherwise $L$ and $R$ would also be connected in the graph $\left(V \backslash U, E_{V \backslash U} \cup F \backslash\{\bar{f}\}\right)$. Hence we can choose $v_{l} \in L$ and $v_{r} \in R$ with $l<r-1$ such that $v_{j} \in V \backslash(L \cup R)=U$ for all $j \in\{l+1, \ldots, r-1\}$. Furthermore, there exists an index $\vec{i}$ such that all vertices $v_{j}$ with $j \in\{l+1, \ldots, r-1\}$ belong to $U_{\bar{s}}$, since $\left\{v_{l+1}, \ldots, v_{r-1}\right\}$ is connected in $\Gamma$. Define $\bar{g}=\left\{v_{l}, v_{r}\right\}$. Now it is clear that the removal of the edge $\bar{f}$ from $\Gamma_{V \backslash U}$ and the addition of $\bar{g}$ to it, results in a tree again, and that $|I(\bar{g})|=1<|I(\bar{f})|$.

Define $G=F \cup\{\bar{g}\} \backslash\{\bar{f}\}$. Then the graph $\left(V \backslash U, E_{V \backslash U} \cup G\right)$ is a tree and $\sum_{g \in G}|I(g)|<\sum_{f \in F}|I(f)|$. According to the induction hypothesis there exists a partition $\left(G_{i}\right)_{i=1}^{k}$ of $G$, such that all graphs $\left(V \backslash U_{i}, E_{V \backslash U_{i}} \cup G_{i}\right)$ are connected.

Assume for a moment that the partition is such that $\vec{g} \in G_{\hat{i}}$ with $\hat{i} \neq \overline{\mathbf{i}}$. The path in $\Gamma$ between the endpoints of $\bar{g}$ does not meet the set $U_{\hat{\mathrm{i}}}$, so this path also exists in the graph $\left(V \backslash U_{\hat{i}}, E_{V \backslash U_{i}}\right)$. It follows that $\bar{g}$ lies on a cycle in the graph $\left(V \backslash U_{\hat{i}}, E_{V \backslash U_{i}} \cup G_{\hat{i}}\right)$. Therefore, $\bar{g}$ can be transfered from $G_{\hat{i}}$ to $G_{\tilde{i}}$, still leaving all graphs $\left(V \backslash U_{i}, E_{V \backslash U_{i}} \cup G_{i}\right)$ connected.

Assume from now on that $\bar{g} \in G_{\bar{i}}$. Define

$$
F_{i}= \begin{cases}G_{i} & \text { if } i \neq \bar{i} \\ G_{\bar{i}} \cup\{\bar{f}\} \backslash\{\bar{g}\} & \text { if } i=\bar{i} .\end{cases}
$$

The graphs $\left(V \backslash U_{i}, E_{V \backslash U_{i}} \cup F_{i}\right)$ are trivially connected for $i \neq \bar{i}$. It remains to prove that $\left(V \backslash U_{\bar{i}}, E_{V \backslash U_{\bar{i}}} \cup F_{\bar{i}}\right)$ is connected. We know that $\left(V \backslash U_{\bar{i}}, E_{V \backslash U_{\bar{i}}} \cup G_{\bar{i}}\right)$ 
is connected. Removal of the edige $\bar{g}=\left\{v_{l}, v_{p r}\right\}$ causes the graph to break into two components. The path from $v_{0}$ to $v_{l}$ in $\Gamma$ uses no vertices of $U_{\bar{i}}$. Hence, in the graph $\left(V \backslash U_{\bar{i}}, E_{V \backslash U_{\bar{i}}} \cup G_{\bar{i}} \backslash\{\bar{g}\}\right)$ the vertices $v_{0}$ and $v_{l}$ lie in the same component. Analogously one shows that $v_{r}$ and $v_{h}$ lie in the same component. It follows that $v_{0}$ and $v_{h}$ lie in different components. Thus, addition of the edge $\bar{f}=\left\{v_{0}, v_{h}\right\}$ results in a connected graph.

Let $V$ be a finite set and let $d$ be a weight function which associates a non-negative number to each pair $\{i, j\}$ with $i, j \in V$. In the following the ordered pair $(V, d)$ will be called a network.

Lemma 4.3 Let $(V, d)$ be a network and let $\Gamma$ be a minimum spanning tree on $V$ in this network. Furthermore, let $U \subseteq V$ and $\left(U, E_{U}\right)$ be the forest which results from $\Gamma$ when all vertices outside $U$ are removed. Then there exists a mest $\Gamma U=(U, F)$ on $U$, such that $E_{U} \subseteq F$.

Proof: Let $T_{U}=(U, \bar{F})$ be a mcst for $U$ with respect to the weight function d. If $E_{U} \subseteq \bar{F}$, or equivalently, if $\left|E_{U} \cap \bar{F}\right|=\left|E_{U}\right|$, then the proof is finished. Therefore assume that $\left|E_{U} \cap \bar{F}\right|=k<\left|E_{U}\right|$. Choose an edge $e \in E_{U} \backslash \bar{F}$. When $e$ is deleted in the tree $\Gamma$, the result is a forest which consists of two components. At least one edge on the path between the endpoints of $e$ in the tree $T_{U}$ connects these two components. Let us denote this edge by $f$. From the minimality of $\Gamma$ it follows that $d_{f} \geq d_{e}$. From the minimality of $T_{U}$ it follows that $d_{c} \geq d_{f}$. Hence, $d_{e}=d_{f}$. Replace the edge $f$ in $T_{U}$ by $e$ and the result is a mest for $U$ which has $k+1$ edges in common with $E_{U}$. The replacement of edges can be repeated to obtain mcst's for $U$ which have $k+2, k+3, \ldots$ edges in common with $E_{U}$. We conclude that there exists a mest on $U$ which has $\left|E_{U}\right|$ edges in common with $E_{U}$.

Theorem 4.4 Let $(N, c)$ be a minimum cost spanning tree game defined on the network $(N \cup\{0\}, d)$, and let $\Gamma=(N \cup\{0\}, E)$ be a mcst for the grand coalition $N$. Then all essential coalitions of the dual game $c^{*}$ are connected in the forest, which results from $\Gamma$ by deleting the common supplier 0 from it.

Proof: Let $S \subseteq N$ and let $S_{1}, S_{2}, \ldots, S_{k}$ denote the components of $S$ with respect to $\Gamma$. Let $\bar{N}=N \cup\{0\}$ and as usual, let $E_{\bar{N} \backslash S} \subseteq E$ denote the set of edges which have both endpoints in $\bar{N} \backslash S$. According to lemma 4.3 it 
is possible to construct a minimal tree $\Gamma_{\bar{N} \backslash S}$ for $\bar{N} \backslash S$ which uses all edges in $E_{\bar{N}} \backslash S$. Equivalently, we can choose a collection of edges $F$, such that $\Gamma_{\bar{N} \backslash S}=\left(\bar{N} \backslash S, E_{\bar{N} \backslash S} \cup F\right)$ is a mcst for $\bar{N} \backslash S$. Hence,

$$
c^{*}(S)=c(N)-c(N \backslash S)=\sum_{e \in E \backslash E_{\bar{N} \backslash S}} d_{e}-\sum_{e \in F} d_{e} .
$$

According to lemma 4.2 there exists a partition $\left(F_{i}\right)_{i=1}^{k}$ of $F$, such that all graphs $\left(\bar{N} \backslash S_{i}, E_{\bar{N} \backslash S_{i}} \cup F_{i}\right)$ are all connected. Hence,

$$
c^{*}\left(S_{i}\right)=c(N)-c\left(N \backslash S_{i}\right) \geq \sum_{e \in E \backslash E_{\bar{N} \backslash s_{i}}} d_{e}-\sum_{e \in F_{i}} d_{e} \text { for all } i \in\{1, \ldots, k\} \text {. }
$$

Observe that $E \backslash E_{\bar{N} \backslash S_{i}}$ is the collection of edges in $\Gamma$ with at least one endpoint, in $S_{i}(i \in\{1, \ldots, k\})$, and $E \backslash E_{\bar{N} \backslash S}$ is the collection of edges in $\Gamma$ with at least one endpoint in $S$. Hence, $\left(E \backslash E_{\bar{N} \backslash S_{i}}\right)_{i=1}^{k}$ forms a partition of $E \backslash E_{\bar{N} \backslash S}$. Now it follows that

$$
\begin{aligned}
c^{*}(S) & =\sum_{e \in E \backslash E_{\bar{N} \backslash S}} d_{e}-\sum_{e \in F} d_{e} \\
& =\sum_{i=1}^{k}\left(\sum_{e \in E \backslash E_{\bar{N} \backslash s_{i}}} d_{e}-\sum_{e \in F_{i}} d_{e}\right) \\
& \leq \sum_{i=1}^{k} c^{*}\left(S_{i}\right) .
\end{aligned}
$$

Thus, $S$ cannot be essential in $c^{*}$ when it consists of more than one component with respect to $\Gamma$. The theorem follows.

Combining theorem 4.4 and theorem 2.8 it follows that the collection of essential coalitions of the dual of a MCST game possesses the TD-property. Also the grand coalition $N$ is stable in the dual game $c^{*}$, since the tree vector lies in its core. Therefore, we can apply theorem 2.12 to generate other vectors in the core of $c^{*}$. That such a procedure really produces other core elements than the tree vector, is shown in the following example.

Example: Consider the graph in figure 4.1 consisting of 4 nodes, the common supplier 0 and three customers, 1,2 and 3. The minimum spanning tree $\Gamma$ contains three edges, $\{0,1\},\{1,2\}$ and $\{2,3\}$. Let $B$ be the collection of coalitions which are connected in $\Gamma$. According to theorem 4.4 all essential coalitions of the dual game $c^{*}$ are contained in $B$. The characteristic functions of $c$ and $c^{*}$ are given in the table below. 


\begin{tabular}{|r||r|r|r|r|r|r|r|}
\hline$S$ & 1 & 2 & 3 & 12 & 13 & 23 & 123 \\
\hline$c$ & 1 & 3 & 4 & 3 & 4 & 5 & 5 \\
\hline$c^{*}$ & 0 & 1 & 2 & 1 & 2 & 4 & 5 \\
\hline
\end{tabular}

$B$ has the TD-property with respect to two different permutations of $N$, namely $(3,2,1)$ and $(1,2,3)$. If we apply theorem 2.12 with respect to the first permutation, we obtain the vector $(1,2,2)$, the tree vector. However, if we apply theorem 2.12 with respect to the second permutation, we obtain the vector $(0,1,4)$, which is also an extreme element of Core $\left(c^{*}\right)$ (and of ACore(c)).

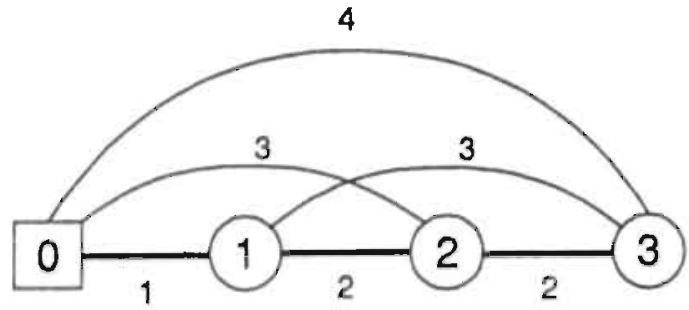

Figure 4.1

In the example above the MCST game has four extreme elements in the anti-core, namely the two vectors found in the example and two other vectors, $(1,1,3)$ and $(0,3,2)$. So even in this simple example we do not find all extreme elements by applying theorem 2.12 . It is worth noting that the procedure offered by theorem 2.12 can be strengthened in the following way for MCST games.

Let $(N, c)$ be a MCST game and suppose that $U \subseteq N$ is a stable coalition in the dual game $c^{*}$. Let $c_{U}^{*}$ denote the subgame of $c^{*}$ restricted to the coalition $U$ and let $c_{N \backslash U}$ denote the subgame of $c$ restricted to the coalition $N \backslash U$. From theorem 4.4 and corollary 2.13 it follows that $c_{U}^{*}$ has a nonempty core. Furthermore, it is trivial that $c_{N \backslash U}$ is a MCST game. Hence, a vector $z=\left(z_{U}, z_{N \backslash U}\right) \in \mathbb{R}^{N}$ with the following properties exists.

Property $4.1 z_{U}$ is a core element of the subgame $c_{U}^{*}$. 


\section{Property $4.2 z_{N \backslash U}$ is a tree vector of the subgame $c_{N \backslash U}$.}

Theorem 4.5 Any vector $z$ which satisfies properties 4.1 and 4.2 is an element of the anti-core of the MCST game $c$ (and an element of Core $\left(c^{*}\right)$ ). Moreover, if $z_{U}$ is chosen extreme in Core $\left(c_{U}^{*}\right)$ then $z$ is extreme in ACore $(c)$.

Proof: Let $\Gamma_{N \backslash U}$ be a minimum spanning tree on the node set $N \backslash U \cup\{0\}$ such that $z_{N \backslash U}$ is the tree vector associated with $\Gamma_{N \backslash U}$. For each player $i \in N \backslash U$, let $f(i)$ denote its follower, i.e. the node which is visited directly after $i$ on the unique path in $\Gamma_{N \backslash U}$ from $i$ to 0 . Hence, for any player $i \in N \backslash U$ we have $z_{i}=d_{i, f(i)}$.

Now, let $S \subseteq N$. We have to show that $z(S) \geq c^{*}(S)$. Therefore, we construct a graph $G$ with node set $\{0\} \cup N \backslash(S \cap U)$ as follows. First, construct a minimal spanning tree on the node set $\{0\} \cup N \backslash S$. Then, add the remaining nodes $S \cap \boldsymbol{N} \backslash U$, together with the edges $\{i, f(i)\}$ for $i \in S \cap N \backslash U$. This graph is well defined, since the endpoints of all edges lie within $\{0\} \cup N \backslash(S \cap U)$. The costs of construction of $G$ equal $z(S \cap N \backslash U)+c(N \backslash S)$. Furthermore, $G$ is a connected graph. To see this, we show that for each $i \in N \backslash(S \cap U)$ there exists a path to 0 . If $i \in N \backslash S$ this follows directly from the fact that $G$ contains a spanning tree on $\{0\} \cup N \backslash S$. If $i \in S \cap N \backslash U$, then follow the path $i, f(i), f(f(i)), \ldots$ until a node in $\{0\} \cup N \backslash S$ is reached. This will surely happen, since the edges $\{i, f(i)\}(i \in S \cap N \backslash U)$ contain no cycle. Once a node in $\{0\} \cup N \backslash S$ is reached, it is trivial again that a path to 0 exists. We conclude that $G$ is connected (in fact, it is a tree). The costs of construction therefore are at least $c(N \backslash(S \cap U))$. Thus, $z(S \cap N \backslash U)+c(N \backslash S) \geq c(N \backslash(S \cap U))$. Using this inequality, we find

$$
\begin{aligned}
c^{*}(S) & =c(N)-c(N \backslash S) \leq \\
& \leq c(N)+z(S \cap N \backslash U)-c(N \backslash(S \cap U))= \\
& =z(S \cap N \backslash U)+c^{*}(S \cap U) \leq \\
& \leq z(S \cap N \backslash U)+z(S \cap U)=z(S) .
\end{aligned}
$$

The efficiency of $z$ follows from $z(N)=z(U)+z(N \backslash U)=c^{*}(U)+c(N \backslash U)=$ $c^{*}(N)$.

Suppose $z_{U}$ is an extreme core element of $c_{U}^{*}$. We shall prove that $z$ is an extreme element in ACore $(c)$. Let $u$ denote the cardinality off $U$ and assume without loss of generality that $U=\{1,2, \ldots, u\}$. Since $z_{U}$ is extreme, we can choose $u$ coalitions $S_{1}, \ldots, S_{u} \subseteq U$ such that $z\left(S_{i}\right)=c_{U i}^{*}\left(S_{i}\right)$ for all $i \in\{1, \ldots, u\}$ and such that the incidence vectors $\bar{B}_{i}$ of these coalitions are linearly independent. We may assume that $S_{u}=U$. Define $T_{i}=N \backslash S_{\mathrm{i}}$ 
for $i \in\{1,2, \ldots, u-1\}$ and define $T_{u}=N$. Then $z\left(T_{i}\right)=c\left(T_{i}\right)$ for $i \in$ $\{1,2, \ldots, u\}$. The tree vector $z_{N \backslash U}$ is extreme in the game $c_{N \backslash U}$. Hence, we can choose coalitions $T_{u+1}, \ldots, T_{n} \subseteq N \backslash U$ such that $z\left(T_{i}\right)=c\left(T_{i}\right)$ for all $i \in\{u+1, \ldots, n\}$ and such that the incidence vectors $t_{i}(i=u+1, \ldots, n)$ are linearly independent.

It will be sufficient to prove that the incidence vectors $t_{i}$ of the sets $T_{i}$ $(i \in\{1,2, \ldots, n\})$ are linearly independent. To see that this is indeed the case, suppose that $\sum_{i=1}^{n} \alpha_{i} t_{i}=0$. After some rearranging we obtain

$$
\sum_{i=1}^{u-1} \alpha_{i} s_{i}-\sum_{i=u+1}^{n} \alpha_{i} t_{i}=1 \sum_{i=1}^{u} \alpha_{i}
$$

where 1 denotes the all-one vector. Observe that the coordinates $u+1, \ldots, n$ of the vectors $s_{i}$ are all 0 , and that the coordinates $1, \ldots, u$ of the vectors $t_{i}$ $(i=u+1, \ldots, n)$ are also 0 . It follows that

$$
\sum_{i=1}^{u-1} \alpha_{i} s_{i}=s_{u} \sum_{i=1}^{u} \alpha_{i}
$$

and that

$$
\sum_{i=u+1}^{n} \alpha_{i} t_{i}=\left(1-s_{u}\right) \sum_{i=1}^{u} \alpha_{i}
$$

Since the vectors $s_{i}(i=1, \ldots, u)$ are linearly indepedent, it follows from (4.2) that $\alpha_{i}=0$ for $i=1, \ldots, u$, and (4.3) can be rewritten as

$$
\sum_{i=u+1}^{n} \alpha_{i} t_{i}=0
$$

Since the vectors $t_{i}(i=u+1, \ldots, n)$ are also linearly independent, it follows that $\alpha_{i}=0$ for $i=u+1, \ldots, n$. We conclude that $t_{1}, \ldots, t_{n}$ are inearly indepedent.

Theorem 4.5 suggests the following approach for generating new (extreme) core elements. Choose a coalition $U \subseteq N$. Use theorem 2.12 to find out whether coalition $U$ is stable in $c^{*}$ and compute an extreme core element $z_{l \prime}$ of $c_{U}^{*}$ if this is the case. Then compute a tree vector $z_{N \backslash U}$ associated with a minimum spanning tree on $\{0\} \cup N \backslash U$. Then $z=\left(z_{U}, z_{N \backslash U}\right)$ is an extreme element of $\mathrm{ACore}(c)$.

Example : (continued) Earlier, we missed the extreme core allocations $(1,1,3)$ and $(0,3,2)$. Using the procedure which we have just sketched, we 
can generate both vectors. Choose $U=\{2\}$. This coalition is trivially stable in $c^{*}$. We charge player 2 with the amount $c^{*}(2)=1$ and we charge players 1 and 3 according to the tree vector with respect to the minimal tree with node set $\{0,1,3\}$. This gives us exactly the missing core element $(1,1,3),(0,3,2)$ can be found by setting $U=\{1\}$.

Still it is not dificult to construct examples in which not all extreme core elements can be found in this way. One of the reasons is that not all coalitions need to be stable in $c^{*}$.

Example : Consider the graph in figure 4.2 consisting of 5 nodes, the common supplier 0 and four customers, 1,2,3 and 4. The minimal spanning tree $\Gamma$ contains four edges, $\{0,1\},\{1,2\},\{2,3\}$ and $\{2,4\}$. The table below contains only the values $c^{*}(S)$ for coalitions $S$, which are connected in $\Gamma$.

\begin{tabular}{|c||r|r|r|r|r|r|r|r|r|r|r|}
\hline$S$ & 1 & 2 & 3 & 4 & 12 & 23 & 24 & 123 & 124 & 234 & $N$ \\
\hline$c^{*}$ & 0 & 0 & 2 & 2 & -4 & 3 & 3 & 2 & 2 & 6 & 8 \\
\hline
\end{tabular}

The vector $(0,0,3,5)$ is an extreme core element of $c^{*}$. However, the procedure sketched in theorem 4.5 will not produce this particular vector, no matter how the set $U$ is chosen. This is easily seen by observing that $U$ should contain both player 1 and player 2. The only stable coalition that contains both players is $N$. The collection of connected coalitions in $\mathrm{r}$ has the TD-property w.r.t. eight different permutations of $N$. In six of them player 2 is the last player in the permutation, and all of these permutations produce the vector $(0,4,2,2)$. The remaining two permutations produce the tree vector $(2,2,2,2)$.

The solution to the problem in this example seems to be simple. We cannot use theorem 4.5 with respect to the set $U=\{1,2,3\}$, because $U$ is not stable in $c^{*}: c^{*}(\{1\})+c^{*}(\{2,3\})=3>2=c^{*}(\{1,2,3\})$. If we simply lower the weight of the edge $\{0,4\}$ from 6 to 5 , we achieve that the dual value of $\{1,2,3\}$ becomes 3 , while the values of other stable coalitions remain unchanged. Hence, the core of the game does not change, but the coalition $\{1,2,3\}$ becomes stable. Now apply theorem 4.5 with respect to the set $\{1,2,3\}$ (permutation: $(1,2,3)$ ), and we obtain the vector $(0,0,3,5)$. Naturally, we wonder whether this approach can be formalized. 


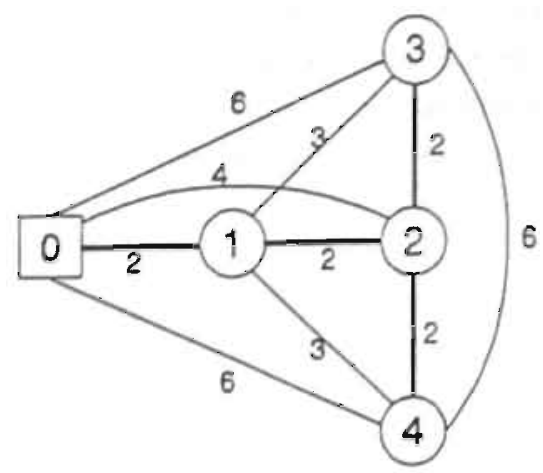

Figure 4.2

In this light it is interesting to discuss briefly the work of Aarts and Driessen in [2] and [1]. In these papers they also investigate the structure of the anti-core of MCST games. Their goal is to deduce elements in the anticore directly from the underlying network, other than the tree vector. Their approach is to lower the weights $d_{i j}$ of establishing a link between $i$ and $j$, such that the anti-core remains unchanged. In general, much computation is needed to verify whether a specific weight reduction of a link will leave the anti-core unchanged. However, Aarts and Driessen propose two types of weight reductions which can be carried out efficiently. We state these results without proof.

Let $(N, c)$ be a MCST game and let $\Gamma$ be a minimum spanning tree with node set $N \cup\{0\}$.

Reduction 1) Let $i, j, k, l$ be such that there is a path in $\Gamma$ from $k$ to $l$ which visits $i$ and $j$. Furthermore, suppose that $d_{i j}>d_{k l}$. Then the weight of the edge $\{i, j\}$ can be lowered from $d_{i j}$ to $d_{k l}$ without changing the anti-core of the game.

Reduction 2) Let $i, j, k, l$ be such that there is a path in $\Gamma$ from $k$ to $l$ which visits $i$ and $j$. Moreover let $i$ and $j$ be adjacent in $\Gamma$ and let $i$ be visited before $j$ on this path. Suppose that $d_{k l}+d_{i j}>d_{k j}+d_{i l}$. Then the weight of the edge $\{k, l\}$ can be lowered from $d_{k l}$ to $d_{k j}+d_{i l}-d_{i j}$ without changing the anti-core of the game.

In the example we actually applied the second weight reduction. We see that $d_{04}+d_{12}=8>7=d_{02}+d_{14}$. The result of Aarts and Driessen 
tells us that we can lower the weight of the edge $\{0,4\}$ from $d_{04}=6$ to $d_{02}+d_{14}-d_{12}=5$ without changing the anti-core of the MCST game. So indeed, the approach of lowering edge weights in order to obtain a greater number of stable coalitions in the dual game without changing its core, can be generalized. However, the following example shows that is unlikely that such an approach allows us to characterize all extreme elements of the core.

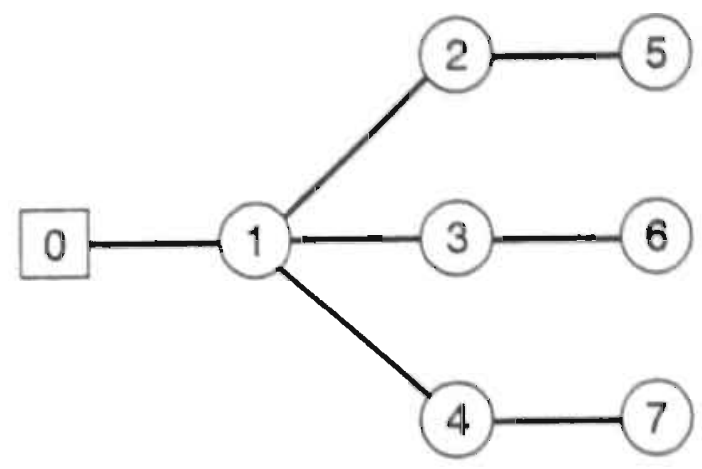

Figure 4.3

Example: Consider the minimum cost spanning tree game with player set $N=\{1,2, \ldots, 7\}$ and minimum cost spanning tree as pictured in figure 4.3. The weights of all edges in this minimal tree are equal to 2. The weights of the other edges are listed in the table below.

\begin{tabular}{|l||c|c|c|c|c|c|c|c|}
\hline & 0 & 1 & 2 & 3 & 4 & 5 & 6 & 7 \\
\hline 0 & - & 2 & 3 & 3 & 3 & 5 & 5 & 5 \\
\hline 1 & - & - & 2 & 2 & 2 & 4 & 4 & 4 \\
\hline 2 & - & - & - & 5 & 5 & 2 & 5 & 4 \\
\hline 3 & - & - & - & - & 5 & 4 & 2 & 5 \\
\hline 4 & - & - & - & - & - & 5 & 4 & 2 \\
\hline 5 & - & - & - & - & - & - & 5 & 5 \\
\hline 6 & - & - & - & - & - & - & - & 5 \\
\hline 7 & - & - & - & - & - & - & - & - \\
\hline
\end{tabular}

Observe that $c(3,5,6)=9$ and $c(N \backslash 3)=c(N)=14$, so for any $x=$ $\left(x_{1}, \ldots, x_{7}\right)$ in the anti-core of $c$ we have $x_{3}+x_{5}+x_{6} \leq 9$ and $x_{3} \geq 0$. We conclude that $x_{5}+x_{6} \leq 9$. Analogously, one proves that $x_{5}+x_{7} \leq 9$ 
and $x_{6}+x_{7} \leq 9$. Adding these inequalities and dividing by 2 gives us $x_{5}+x_{6}+x_{7} \leq 27 / 2$. The last constraint turns out to be binding, i.e. elements in the anti-core exist which satisfy $x_{5}+x_{6}+x_{7}=27 / 2$. It is a matter of verification that $(1 / 2,0,0,0,9 / 2,9 / 2,9 / 2)$ is an extreme element of the anti-core of the game.

The example above is constructed such that no edge weight can be lowered without changing the anti-core of $c$. Since all edge weights are integers, it follows that the values of all coalitions are integers too. Therefore, our procedure to generate extreme elements of the anti-core will only produce integral vectors and hence, the fractional vector $(1 / 2,0,0,0,9 / 2,9 / 2,9 / 2)$ will not be found in this way.

\subsection{Information graph games}

\subsubsection{Introduction}

In the previous section we have provided a method for constructing many extreme elements of the anti-core of a MCST game. We have seen that this method does not characterize all extreme elements, and to our knowledge, no such characterization of the extreme elements exists.

The anti-nucleolus of a MCST game can be computed using the algorithm described in section 2.6. Although the computational savings of this algorithm are considerable compared to a general purpose algorithm, the algorithm is still not polynomial in the number players of the game. To our knowledge no polynomial time algorithm exists for computing the antinucleolus of a MCST game.

However, for some subclasses of MCST games and monotonic MCST games the results are more satisfactory. Earlier we discussed monotonic MCST games. These games are not necessarily defined on a complete weighted graph, but they can also be defined on any connected graph. Megiddo[36] considers the case where the underlying graph is a tree. He shows that in this case it is possible to compute the Shapley value in $O(n)$ elementary operations and the anti-nucleolus in $O\left(n^{3}\right)$ elementary operations, where $n$ is the number of players in the game. In this section we restrict ourselves to a subclass of MCST games, called information graph games. In this case a full characterization of the extreme elements of the anti-core is possible and also the anti-nucleolus can be computed efficiently.

Given is a set of customers $N$ who are all interested in a particular 
piece of information, e.g. a computer program. A subset $Z$ of $N$, called the informed set, already possesses this information. Other customers may purchase the information from a supplier for a fixed price, say 1, or they may share the information with a friendly customer, who already has the information. These friendly relations between customers are stored in an undirected graph $G=(\boldsymbol{N}, E)$, called the information graph. Players $i$ and $j$ can send information to each other if and only if $\{i, j\} \in E$.

Suppose a subset of customers $S \subseteq N$ decides to form a coalition. Assume that the customers in $S$ all need to get informed and that they do not seek the cooperation of customers outside $S$ in order to achieve this goal. Consider the graph $G_{S}$ that results from $G$ by deleting all vertices (customers) outside $S$ and all edges with at least one endpoint outside $S$. Customers within one component of $G_{S}$ can freely share their information. So, if this component contains a customer in the informed set, then the cost to get all customers of this component informed equals 0 . Otherwise, one of the customers in this component will have to purchase the information from the supplier at cost 1 and then share it with the other customers in the component. Thus, the cost to get all players of $S$ informed is equal to the number of components of $G_{S}$ that have no customer in the informed set. We denote this cost by $c(S)$. The cooperative cost allocation game with player set $N$ and characteristic function $c$ is called an information graph game.

One may also view information graph games as a subclass of minimum cost spanning tree games. This is seen as follows. Let $(N, c)$ be an information graph game with informed set $Z$ and information graph $G=(N, E)$. For a pair $i, j \in N$ define $d_{i j}=0$ if $\{i, j\} \in E$ and $d_{i j}=1$ otherwise. Furthermore, define $d_{i 0}=0$ if $i \in Z$ and $d_{i 0}=1$ otherwise. Now, observe that the characteristic function of the MCST game defined on the complete graph with vertex set $N \cup\{0\}$ and weight function $d$ equals $c$.

\subsubsection{Concave information graph games}

Let $(N, c)$ be a cooperative cost allocation game. The game $c$ is said to be concave if

$$
c(S)+c(T) \geq c(S \cap T)+c(S \cup T)
$$

for all $S, T \subseteq N$.

In this section we give necessary and sufficient conditions on the information graph and the informed set, such that the associated information graph game is concave. First we need some preliminary definitions. 
Let $G=(V, E)$ be an undirected graph. $G$ is called 2-connected if it is connected and if removing one vertex, no matter which one, leaves the remainder of the graph connected. For convenience we shall say that a set $U \subseteq V$ is connected, 2-connected, complete, etc., if the graph $G_{U}$, which results from $G$ by deleting all vertices outside $U$, has this property.

The maximal 2-connected subsets of a graph $G$ are called the blocks of $G$.

We call a graph cycle complete (see van den Nouweland and Borm[41] and van den Nouweland[40]) if every for cycle in the graph the subgraph on the vertices in this cycle is a complete graph. In chapter 6 we also use the properties of cycle complete graphs. There it is proved in lemma 6.14 that the collection of connected coalitions of a cycle complete graph is closed under taking intersections. This result also follows from theorem 2.2.3 in van den Nouweland $[40]$. We shall use that result here too.

Lemma 4.6 Let. $G \equiv(V, E)$ be a cycle complete graph and let $S, T \subseteq V$ with components $S_{1}, \ldots, S_{k}$ and $T_{1}, \ldots, T_{l}$ respectively. Furthermore, let $\mathcal{G}$ be the bipartite graph with node set $\mathcal{V}=\left\{S_{1}, \ldots, S_{k}\right\} \cup\left\{T_{1}, \ldots, T_{l}\right\}$ and edge set $\mathcal{E}=\left\{\left\{S_{i}, T_{j}\right\} \mid S_{i} \cup T_{j}\right.$ is connected $\}$. Then $\mathcal{G}$ is a forest.

Proof: Suppose that $g$ is not a forest. Then let $\left(U_{1}, \ldots, U_{n}, U_{1}\right)$ (with $n \geq 3$ ) be a cycle in $G$.

Choose $u_{i} \in U_{i}$ for $i=1, \ldots, n$. Since $U_{i} \cup U_{i+1}$ is connected for $i=$ $1, \ldots, n-1$, there exists a path in $G$ from $u_{i}$ to $u_{i+1}(i=1, \ldots, n-1)$. Also, there exists a path in $G$ from $u_{n}$ to $u_{1}$. After concatenation of these paths we obtain a path in the graph $G$, which visits all sets $U_{i}$, and which has $u_{1}$ as its initial and final vertex. Let us denote this path by $P$. The path $P$ is not necessarily a cycle, because it may visit some vertices more than once. However, a cycle which visits at least three of the sets $U_{i}$ can be constructed from $P$ as follows.

If $P$ is not a cycle, then it contains a sequence of vertices $\left(v_{1}, \ldots, v_{k}, v_{1}\right)$ for which only the initial and final vertex coincide. (It is not necessarily a cycle, perhaps the sequence contains only two different vertices.) If this sequence visits at least three of the sets $U_{i}$, then stop: we have found a cycle which visits at least three of the sets $U_{i}$. Otherwise, contruct a new path $\bar{P}$ by replacing the sequence $\left(v_{1}, \ldots, v_{k}, v_{1}\right)$ in $P$ by its initial (and final) vertex $v_{1}$. The path $\bar{P}$ still visits all sets $U_{1}, \ldots, U_{n}$. Let us make this clear. If the sequence $\left(v_{1}, \ldots, v_{k}, v_{1}\right)$ visits only one set $U_{i}$, then this is trivial. Thus suppose that the sequence visits precisely two of the sets $U_{i}$. Obviously, 
these sets must be consecutive. Without loss of generality assume that the sequence visits $U_{1}$ and $U_{2}$ and that its initial vertex $v_{1}$ lies in $U_{1}$. The path $\bar{P}$ still visits $U_{1}$ because it contains $v_{1} \in U_{1}$. The path $\bar{P}$ also visits $U_{2}$ since otherwise the path would jump from a vertex in $U_{1}$ to a vertex in $U_{3}$, contradicting the fact that $U_{1}$ and $U_{3}$ are either both components of $S$ or both components of $T$. Repeat the process until a cycle is found which visits at least three of the sets $U_{i}$ or until the path $P$ has become a cycle which visits all of the sets $U_{i}$.

We have found a cycle in $G$ which visits at least three of the sets $U_{i}$. Without loss of generality we assume that at least two of these sets are components of $S$, say $U_{l}$ and $U_{k}$. Choose a vertex $v_{l} \in U_{l}$ which lies on the cycle, and a vertex $v_{k} \in U_{k}$ on the cycle. From the cycle completeness of $G$ it follows that $\left\{v_{l}, v_{k}\right\} \in E$. Hence $U_{l} \cup U_{k}$ is a connected set, contradicting the fact that $U_{l}$ and $U_{k}$ are components of $S$. We conclude that the graph $\mathcal{G}$ contains no cycles. In other words, $\mathcal{G}$ is a forest.

The following theorem gives necessary and sufficient conditions on the informed set and the information graph, such that the corresponding information graph game is concave.

Theorem 4.7 Let $(N, c)$ be an information graph game with information graph $G=(N, E)$ and informed set $Z$. Then $c$ is concave if and only if $G$ and $Z$ satisfy the following properties.

i) Each elementary path with its initial and final vertex in $Z$ is contained in $Z$.

ii) Each cycle, whose intersection with $Z$ contains at most one element, is complete.

Proof: To prove the 'only if'-part suppose that $c$ is concave. Let $\left(v_{1}, \ldots, v_{k}\right)$ be an elementary path with $v_{1}, v_{k} \in Z$. Let $i$ be such that $1<i<k$. Define. $S=\left\{v_{1}, \ldots, v_{i}\right\}$ and $T=\left\{v_{i}, \ldots, v_{k}\right\}$. Then $S, T$ and $S \cup T$ are connected and contain at least one element of $Z$. Therefore $c(S)=c(T)=c(S \cup T)=0$. From the concavity of $c$ it follows that $c(S \cap T)=c\left(\left\{v_{i}\right\}\right)=0$ and thus $v_{i} \in Z$. This proves that $Z$ satisfies property i).

Let $U \subseteq N$ be a cycle whose intersection with $Z$ contains at most one element. Choose $i, j \in U$. Clearly there exist two vertex-disjoint paths in $U$ from $i$ to $j$, say $\left(i, v_{1}, \ldots, v_{k}, j\right)$ and $\left(i, w_{1}, \ldots, w_{l}, j\right)$. Define $S=$ $\left\{i, v_{1}, \ldots, v_{k}, j\right\}$ and $T=\left\{i, w_{1}, \ldots, w_{l}, j\right\}$. Consider three possibilities. a) 
Neither $S$ nor $T$ contains an element of $Z$. b) Precisely one of the coalitions $S$ and $T$ contains an element of $Z$. c) Both $S$ and $T$ contain the unique element of $Z \cap U$. In all three cases it is easily verified that $\{i, j\} \notin E$ implies $c(S \cap T)+c(S \cup T)>c(S)+c(T)$. This contradicts the concavity of $c$, so it follows that $\{i, j\} \in E$. This proves that $G$ and $Z$ satisfy property ii).

To prove the 'if'-part suppose that $G$ and $Z$ satisfy properties i) and ii). Suppose that $S$ forms ar cycle, which is not complete. Then it must contain at least 2 vertices in $Z$, say $z_{1}$ and $z_{2}$. Let $i \in S$. Clearly, an elementary path from $z_{1}$ to $z_{2}$ exists that contains $i$. And hence, $i \in Z$. It follows that $S \subseteq Z$. Now it is clear that the characteristic function $c$ does not change if an edge is added between two vertices in $S$. Therefore we may assume that all cycles are complete.

Let $S, T \subseteq N$. Denote the components of $S$ by $S_{1}, \ldots, S_{k}$ and the components of $T$ by $T_{1}, \ldots, T_{l}$. Construct the graph $\mathcal{G}=(\mathcal{V}, \mathcal{E})$ as in lemma 4.6, i.e. $\mathcal{V}=\left\{S_{1}, \ldots, S_{k}\right\} \cup\left\{T_{1}, \ldots, T_{l}\right\}$ and $\mathcal{E}=\left\{\left\{S_{i}, T_{j}\right\} \mid S_{i} \cup T_{j}\right.$ is connected $\}$. According to lemma 4.6 this graph is a forest. Therefore, the number of edges equals the number of vertices minus the number of components, i.e.

$$
|\mathcal{E}|=k(S)+k(T)-k(S \cup T)
$$

where $k(S), k(T)$ and $k(S \cup T)$ denote the number of components of $S, T$ and $S \cup T$ respectively.

Let $\mathcal{G}_{Z}=\left(\mathcal{V}_{Z}, \varepsilon_{Z}\right)$ be the graph that results from $\mathcal{G}$ when all vertices $S_{i}$ and $T_{j}$ are removed that have empty intersection with $Z$. This graph is also a forest and its number of edges therefore equals the number of vertices minus the number of components. $c(S)+c(T)$ vertices have been removed, so the graph $g_{z}$ has $k(S)-c(S)+k(T)-c(T)$ vertices. It follows from property i) that within each component of $\mathcal{G}$ the vertices that have a nonempty intersection with $Z$ form a connected set in $\mathcal{G}$. Thus, the number of components that have been removed equals $c(S \cup T)$ and it follows that the graph $\mathcal{G}_{Z}$ has $k(S \cup T)-c(S \cup T)$ components. It follows that

$$
|\mathcal{E}|-\left|\mathcal{E}_{Z}\right|=c(S)+c(T)-c(S \cup T)
$$

Furthermore, if $S_{i} \cap T_{j} \neq \emptyset$ and $S_{i} \cap T_{j} \cap Z=\emptyset$ then it follows from property i) that $S_{i} \cap Z=\emptyset$ or $T_{j} \cap Z=\emptyset$. Using these results the concavity of $c$ now 


\section{follows from}

$$
\begin{array}{rlrl}
c(S \cap T)= & c\left(\left(\cup_{i=1}^{k} S_{i}\right) \cap\left(\cup_{j=1}^{l} T_{j}\right)\right) \\
= & & c\left(\cup_{i, j}\left(S_{i} \cap T_{j}\right)\right) \\
\leq & & \sum_{i, j} c\left(S_{i} \cap T_{j}\right) \\
= & & \mid\left\{(i, j) \mid S_{i} \cap T_{j} \neq \emptyset \text { and } S_{i} \cap T_{j} \cap Z=\emptyset\right\} \mid \\
= & & \mid\left\{(i, j) \mid S_{i} \cap T_{j} \neq \emptyset \text { and }\left(S_{i} \cap Z=\emptyset \text { or } T_{j} \cap Z=\emptyset\right)\right\} \mid \\
\leq & & \mid\left\{(i, j) \mid S_{i} \cup T_{j} \text { connected and }\left(S_{i} \cap Z=\emptyset \text { or } T_{j} \cap Z=\emptyset\right)\right\} \mid \\
= & & \mid\left\{(i, j) \mid S_{i} \cup T_{j} \text { connected }\right\} \mid- \\
& & \mid\left\{(i, j) \mid S_{i} \cup T_{j} \text { connected and } S_{i} \cap Z \neq \emptyset \text { and } T_{j} \cap Z \neq \emptyset\right\} \mid \\
= & & |\mathcal{E}|-\left|\mathcal{E}_{Z}\right| \\
= & & c(S)+c(T)-c(S \cup T) .
\end{array}
$$

Theorem 4.7 is closely related to a theorem of van den Nouweland and Borm[41]. In that paper they consider so calied communication situations. A communication situation is a triple $(N, v, E)$, where $(N, v)$ is a game and $(N, E)$ is an undirected graph. The worth $v(S)$ of a coalition $S$ represents the potential profit of $S$, provided that all players in $S$ can cooperate. The graph $(N, E)$ represents the communication possibilities of players. Players $i$ and $j$ can communicate with each other if and only if $\{i, j\} \in E$. In the commumication model it is assumed that two groups of players cannot cooperate if they cannot communicate. This assumption gives rise to a new game, the so called graph restricted game $v_{E}$, in which the limited possibilities for cooperation are reflected. Let $S \subseteq N$ and let $S_{1}, \ldots, S_{k}$ denote the components of $S$ with respect to the graph $(N, E)$. The worth of coalition $S$ in the graph restricted game $v_{E}$ is defined by

$$
v_{E}(S)=\sum_{i=1}^{k} v\left(S_{i}\right) .
$$

The main result of van den Nouweland and Borm is stated in the following theorem.

Theorem 4.8 Let $(N, v, E)$ be a communication situation where the game $(N, v)$ is convex and the graph $(N, E)$ is cycle complete. Then the corresponding graph restricted game $\left(N, v_{E}\right)$ is convex.

Theorem 4.8 is still valid when the term convex is replaced by concave. Using theorem 4.8 it is possible to give an alternative proof of the 'if'-part of theorem 4.7 in case $|Z| \leq 1$. We give a short sketch of the proof. 
Let $N$ be a set of players, let $Z \subseteq N$ with $|Z| \leq 1$ and let $(N, E)$ be an undirected graph. Define the game $(N, c)$ by

$$
c(S)= \begin{cases}0 & \text { if } S \cap Z \neq \emptyset \\ 1 & \text { if } S \cap Z=\emptyset .\end{cases}
$$

Observe that the game $c$ is concave. Furthermore, the graph restricted game $c_{E}$ is precisely the information graph game with information graph $(N, E)$ and informed set $Z$. Now, suppose $Z$ and $(N, E)$ satisfy the properties of theorem 4.7. It follows from property ii) in theorem 4.7 that the graph $(N, E)$ is cycle complete. Now we can prove the 'if'-part of theorem 4.7 by applying theorem 4.8 to deduce that $c_{E}$ is concave. When $|Z| \geq 2$ a proof along these lines fails, since then the game $c$ defined above is not concave.

At first glance, it is not obvious that the conditions in theorem 4.7 can be verified efficiently, since the number of cycles and the number of paths in a graph may be exponential in the number of vertices of the graph. However, an efficient check is possible. This will be shown in the following. The next lemma can be found in many text books on graph theory and is therefore stated without proof (see e.g. Berge[4]).

Lemma 4.9 (Menger, 1926). A graph is 2-connected if and only if each pair of distinct vertices can be joined by two vertex-disjoint paths.

The conditions in theorem 4.7 are equivalent to the conditions in the following corollary of the theorem.

Corollary 4.10 Let $(N, c)$ be an information graph game with information graph $G$ and informed set $Z$. Then $c$ is concave if and only if $G$ and $Z$ satisfy the following properties.

i) For each component $K$ of the information graph $G$ we have that $K \cap Z$ is connected.

ii) Each block of $G$ is contained in $Z$ or it has at most one element in common with $Z$.

iii) Each block, whose intersection with $Z$ contains at most one element, is complete.

Proof: We shall prove that the conditions in theorem 4.7 are equivalent to the conditions in this corollary. In this proof we shall denote the two conditions in theorem 4.7 by $\mathrm{Ti}$ ) and $\mathrm{Tii}$ ). The three conditions in this 
corollary are denoted by Ci), Cii) and Ciii). First suppose that Ti) and Tii) hold. We have to to prove that $\mathrm{Ci}$ ), Cii) and Ciii) hold. Ci) follows directly from $\mathrm{Ti}$ ). To prove that $\mathrm{Cii}$ ) holds, let $B$ be a block which contains at least 2 elements of $Z$, say $i$ and $j$. We have to prove that $B \subseteq Z$. Choose $k \in B$. According to lemma 4.9 there exist two vertex-disjoint paths from $i$ to $k$ that use only vertices in $B$. At least one of these paths does not contain $j$, so there exists a path in $B$ from $i$ to $k$ not containing $j$. Furthermore, there exist two vertex-disjoint paths in $B$ from $k$ to $j$. These paths may all be taken elementary. Follow the path from $i$ to $k$ until it hits one of the paths from $j$ to $k$. From here, follow this path until the vertex $k$ is reached. Then finally, follow the second path from $j$ to $k$ backwards. This way we have constructed an elementary path from $i$ to $j$ which contains the vertex $k$. It follows directly from $\mathrm{Ti}$ ) that $k \in Z$. Since $k \in B$ was chosen arbitrarily, we conclude that $B \subseteq Z$.

To prove that Ciii) holds, suppose that $B$ is a block which contains at most one element in $Z$. Choose $i, j \in B$. There exist two vertex-disjoint paths from $i$ to $j$ in $B$, and these paths may be taken elemetary. Hence, the vertices on these paths form a cycle which contains at most one element of $Z$. It follows from Tii) that $i$ and $j$ are adjacent. Since $i$ and $j$ were chosen arbitrarily, it follows that $B$ is complete.

Now assume that $\mathrm{Ci}$ ), $\mathrm{Cii}$ ) and $\mathrm{Ciii}$ ) hold. We shall prove that $\mathrm{Ti}$ ) and Tii) hold. We prove Ti) using an inductive argument. Let $i, j \in Z$, and suppose that $p(i, j)$ is an elementary path from $i$ to $j$. If the path $p(i, j)$ consists of only two vertices, namely $i$ and $j$, then the path is trivially contained in $Z$, so in this case Ti) holds. Assume that $p(i, j)$ contains $k$ vertices $(k \geq 3)$, and assume that $\mathrm{Ti}$ ) holds if the path contains less than $k$ vertices. First assume that $i$ and $j$ are the only elements of $Z$ on the path $p(i, j)$. Clearly, $i$ and $j$ lie in one component of the information graph. Let us denote this component by $K$. According to $\mathrm{Ci}$ ) the set $K \cap Z$ is connected. This means that there exist an elementary path $q(i, j)$ from $i$ to $j$ that uses only vertices in $Z$. Thus, the paths $q(i, j)$ and $p(i, j)$ are vertex-disloint, and the vertices on these two paths form a cycle. Since a cycle is a 2-connected set, there must be a block, say $B$, that contains the cycle. It follows from $C$ ii) that $B \subseteq Z$, since $B$ contains more than one element of $Z$. This contradicts the fact that $i$ and $j$ were the only elements of $Z$ on the path $p(i, j)$. So we may assume that the path $p(i, j)$ contains another vertex in $Z$ besides $i$ and $j$, say $k$. Now apply the induction hypothesis twice, once to the path from $i$ to $k$, and once to the path from $k$ to $j$. It follows that the complete path $p(i, j)$ is contained in $Z$. 
Finally, Tii) follows directly from Ciii).

There exist algorithms to determine all blocks of a graph, which are linear in the number of edges of the graph (see Tarjan [58]). Therefore, it is also possible to verify the conditions in corollary 4.10 efficiently.

\subsubsection{A description of the anti-core by means of linear con- straints}

Let $(N, c)$ be an information graph game with information graph $G=(N, E)$ and informed set $Z$. Assume for the moment that the graph $G$ is connected. Let $G_{i}$ denote the graph that results from $G$ if vertex $i$ and all edges with $i$ as an endpoint are deleted. Furthermore, let $K_{i 1}, \ldots, K_{i k_{i}}$ denote the vertex sets of the components of $G_{i}$. Let us denote the collection of coalitions $K_{i j}$ over all graphs $G_{i}$ by $K$. Of course, $\operatorname{ACore}(c)$ is contained in the set $C(c)$ described by

$$
\left\{\begin{array}{l}
x(N)=c(N) \\
x(K) \leq c(K) \text { for all } K \in K .
\end{array}\right.
$$

In fact we have the following theorem.

Theorem 4.11 The set $C(c)$ equals ACore $(c)$. Furthermore, the number of constraints in the description of $C(c)$ is at most $2 n-1$.

Let us first prove that the number of constraints that describe $C(c)$ is bounded by $2 n-1$. Suppose that we remove an edge from the graph $G$, such that the resulting graph remains connected. Clearly, this can only increase the number of components of the graphs $G_{i}$. Thus, we get the maximum number of restrictions when no edge of $G$ can be deleted without disturbing its connectedness, i.e. when $G$ is a tree. Therefore, in deriving an upper bound for the number of restrictions, we may assume that $G$ is a tree. Consequently, the graphs $G_{i}$ are all forests. Let us denote the number of edges in $G_{i}$ by $e_{i} . k_{i}$ denotes the number of components of $G_{i}$ and the number of vertices of $G_{i}$ is $n-1$. Thus we have

$$
k_{i}=n-1-e_{i} .
$$

Suppose that $\left\{i_{1}, i_{2}\right\}$ is an edge of the graph $G$. It is clear that this edge is an edge in all graphs $G_{i}$, except in $G_{i_{1}}$ and in $G_{i_{2}}$. Thus,

$$
\sum_{i=1}^{n} e_{i}=(n-2)(n-1)
$$


since each edge of $G$ is counted $n-2$ times and there are $n-1$ edges in $G$. So it follows that

$$
\sum_{i=1}^{n} k_{i}=n(n-1)-\sum_{i=1}^{n} e_{i}=2(n-1) .
$$

I.e. the number of inequalities in the description of $C(c)$ is precisely $2(n-1)$ when $G$ is a tree. We have one extra equality $x(N)=c(N)$, which makes a total of exactly $2 n-1$ constraints when $G$ is a tree. When $G$ contains cycles, there are even less constraints.

Now we have to prove that $\operatorname{ACore}(c)=C(c)$, i.e. we have to prove that each constraint of the form $x(S) \leq c(S)$ is implied by the constraints of $C(c)$.

Let $S \subseteq N$. First suppose that $S$ is connected. Let us say that a vertex $v \notin S$ separates the vertex $u \neq v$ from $S$, if all paths with initial vertex $u$ and final vertex in $S$ contain the vertex $v$. Equivalently, the vertex $u$ and the set $S$ lie in different components of the graph $G_{v}$. Define

$$
U:=\{u \in N \backslash S \mid \text { no vertex in } N \backslash S \text { can separate } u \text { from } S\} .
$$

We then have the following lemma.

Lemma 4.12 For each vertex $v \in N \backslash(U \cup S)$ there is precisely one vertex $u \in U$ that separates $v$ from $S$.

Proof: Suppose $v \in N \backslash(U \cup S)$. We first prove that at least one vertex $u \in U$ separates $v$ from $S$. By definition there exists a vertex $u_{0} \in N \backslash S$ that separates $v$ from $S$. If $u_{0} \in U$ then the existence part of the proof is finished. If not, choose $u_{1} \in N \backslash S$ that separates $u_{0}$ from $S$. Obviously, $u_{1}$ also separates $v$ from $S$. Continue this process until finally a vertex $u_{k} \in U$ is found, which separates $v$ (and $u_{0}, \ldots, u_{k-1}$ ) from $S$. We shall prove now that at most one vertex $u \in U$ separates $v$ from $S$. Suppose that $u, w \in N \backslash S$ $(u \neq w)$ both separate $v$ from $S$. Then each path from $v$ to $S$ contains both $u$ and $w$. On such a path the vertices $u$ and $w$ are always visited in a fixed order, since otherwise it would be possible to construct a path from $v$ to $S$ with only one of the vertices $u$ or $w$. Assume that $w$ is always visited before $u$. Then $u$ separates $w$ from $S$ and therefore $w$ cannot be an element of $U$. It follows that $U$ contains at most one element that separates $v$ from $S$. The existence of such an element was already proved, which shows that there is precisely one element in $U$ that separates $v$ from $S$. 
For each $u \in U$ let $K_{u}$ denote the component of $G_{u}$ that contains $S$ and let $x \in \mathbb{R}^{N}$. Using lemman 4.12, we obtain the following equality.

$$
\sum_{u \in U} x\left(K_{u}\right)=(|U|-1) x(N)+x(S) .
$$

This is explained as follows. Let $i$ be a player in $S$. Then by definition $i \in K_{u}$ for all $u \in U$. Thus, the variable $x_{i}$ is counted $|U|$ times on the left hand side of the equation. Trivially, $x_{i}$ is also counted $|U|$ times on the right hand side.

Now suppose that $i \in U$. Trivially, $i$ is not an element of $K_{i}$ and since $i$ cannot be separated from $S$ it is an element of $K_{u}$ for all $u \neq i$. Thus the variable $x_{i}$ is counted $|U|-1$ times on the left hand side and also $|U|-1$ times on the right hand side of the equation.

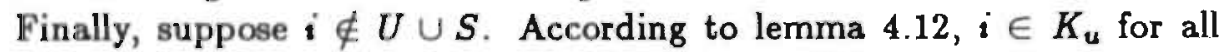
$u \in U$ except one. Thus the variable $x_{i}$ is counted $|U|-1$ times on the left hand side and also $|U|-1$ times on the right hand side. We conclude that equation (4.5) is correct.

The coalitions $K_{\mathrm{u}}$ are all members of the collection $K$. Together with the constraint $x(N)=c(N)$, they imply

$$
x(S)=\sum_{u \in U} x\left(K_{u}\right)-(|U|-1) x(N) \leq \sum_{u \in U} c\left(K_{u}\right)-(|U|-1) c(N) .
$$

It remains to prove that

$$
\sum_{u \in U} c\left(K_{u}\right)-(|U|-1) c(N) \leq c(S) .
$$

We distinguish three cases.

i) The informed set $Z$ is empty.

ii) $S$ contains an element of $Z$.

iii) The remaining case, i.e. $Z \neq \emptyset$ and $Z \cap S=\emptyset$.

Let us first consider case i). If the informed set is empty, then all connected sets have a cost equal to 1 . Recall that $G$ is still assumed to be connected. Thus,

$$
\sum_{u \in U} c\left(K_{u}\right)-(|U|-1) c(N)=|U|-(|U|-1)=1=c(S)
$$


Case ii): In this case all connected sets containing an element of $Z$ have a cost equal to 0 . Thus,

$$
\sum_{u \in U} c\left(K_{u}\right)-(|U|-1) c(N)=0=c(S) .
$$

Case iii): Let $z \in Z$. Since $z \notin S$, it follows from lemma 4.12 that $z$ is an element of precisely $|U|-1$ of the sets $K_{\mathrm{u}}$. These $|U|-1$ components therefore have a cost equal to 0 . The remaining component can have a cost of at most 1. Thus,

$$
\sum_{u \in U} c\left(K_{u}\right)-(|U|-1) c(N) \leq 1=c(S) .
$$

We have proved that the constraints of $C(c)$ imply all constraints of the form $x(S) \leq c(S)$ for connected coalitions $S$. Let us suppose therefore that $S$ is not connected. Let $S_{1}, S_{2}, \ldots, S_{k}$ denote the components of $S$. We have just proved that the constraints $x\left(S_{i}\right) \leq c\left(S_{i}\right)$ are all implied by the constraints of $C(c)$. And thus,

$$
x(S)=\sum x\left(S_{i}\right) \leq \sum c\left(S_{i}\right)=c(S)
$$

is also implied by these constraints.

We conclude that ACore $(c)=C(c)$. Up to this moment it was assumed that the information graph $G$ was connected. If this is not the case then one can deal with each component of $G$ separately, because the anti-core of the game associated with $G$ is the Cartesian product of the anti-cores associated with the components of $G$.

Here the proof of theorem 4.11 ends.

\subsubsection{The extreme elements of the anti-core}

In this subsection we shall use the results of subsections 4.3 .2 and 4.3 .3 to prove that each information graph game has an associated concave information graph game, which has the same anti-core as the original game. This result is implied by two theorems, that tell us how to adjust the information graph and the informed set of an information graph game without changing its anti-core. After a finite number of adjustments we end up with a concave information game which has the same anti-core as the original game. This gives us a nice characterization of the extreme elements of the anti-core of an information graph game, since these are precisely the marginal allocation 
vectors of the associated concave game (see Shapley[49]). Also, every extreme vector in the anti-core is a marginal allocation vector in the original game, though not all marginal allocation vectors need to be elements of the anti-core.

Theorem 4.13 Let $(N, c)$ be an information graph game with information graph $G=(N, E)$ and informed set $Z$. Suppose there is a player $i \in N \backslash Z$ and an elementary path from one vertex in $Z$ to another vertex in $Z$ that contains player $i$. Let $\hat{c}$ be the information graph game with the same information graph $G$ and informed set $\hat{Z}=Z \cup\{i\}$. Then $A \operatorname{Core}(c)=A \operatorname{Core}(\hat{c})$.

Proof : Suppose $K$ is a component of the graph $G_{j}(j \in N)$. If $i \notin$ $K$ then trivially $c(K)=\hat{c}(K)$. Thus suppose $i \in K$. There exists an elementary path with both endpoints in $Z$ containing player $i$. The removal of $j$ from the graph $G$ can separate $i$ from at most one of these endpoints in $Z$. Equivalently, at least one of these two points in $Z$ also lies in $K$, so $c(K)=\hat{c}(K)=0$. We also have $c(N)=\hat{c}(N)$, so, using theorem 4.11, it follows directly that

$$
\operatorname{ACore}(\hat{c})=C(\hat{c})=C(c)=\operatorname{ACore}(c) .
$$

Also, the information graph of an information graph game can be adjusted without changing the anti-core. This is expressed in the following theorem.

Theorem 4.14 Let $(N, c)$ be an information graph game with information graph $G=(N, E)$ and informed set $Z$. Suppose $i, j \in N$ are non-adjacent players contained in a cycle $S \subseteq N$. Let $\hat{c}$ be the information graph game with information graph $\hat{G}=(N, E \cup\{\{i, j\}\})$ and informed set $Z$. Then $A \operatorname{Core}(\hat{c})=A \operatorname{Core}(c)$.

Proof: Let $u \in N$. We shall first prove that the components of $G_{u}$ and $\hat{G}_{u}$ are the same. To this end we show that $v$ and $w$ lie in one component of $\hat{G}_{u}$ if and only if they lie in one component of $G_{u}$. To prove the 'if'-part suppose that $v$ and $w$ lie in one component of $G_{u}$. Then apparently there exists a path from $v$ to $w$ in the graph $G$ that does not use the vertex $u$. This path also exists in $\hat{G}$, since the edge set of $\hat{G}$ contains the edge set of $G$ as a subset. Therefore, $v$ and $w$ lie in one component of the graph $\hat{G}_{u}$. 
To prove the 'only if'-part suppose that $v$ and $w$ lie in one component of $\hat{G}_{u}$. Then there exists a path from $v$ to $w$ in $\hat{G}$ that does not use the vertex $u$. Suppose this path uses the extra edge $\{i, j\}$. Since the vertices $i$ and $j$ lie on a cycle there exist two vertex-disjoint paths from $i$ to $j$ in the graph $G$. At least one of these paths does not use the vertex. $u$. Use this path to get from $i$ to $j$ instead of the edge $\{i, j\}$. Thus, we have constructed a path from $v$ to $w$ that does not use vertex $u$ in the graph $G$. Therefore, $v$ and $w$ lie in one component of $G_{u}$. It follows that the components of $\hat{G}_{u}$ and $G_{u}$ are the same.

Obviously, also the costs of these components is in both cases the same. Using theorem 4.11 it follows immediately that

$$
\operatorname{ACore}(\hat{c})=C(\hat{c})=C(c)=\operatorname{ACore}(c) .
$$

From theorems $4.7,4.13$ and 4.14 we deduce

Corollary 4.15 Let $(N, c)$ be an information graph game with information graph $G=(N, E)$ and informed set $Z$. Let $\hat{Z}$ be the set of all vertices that lie on an elementary path with both endpoints in $Z$ and let $\hat{E}$ be the set of all pairs $\{i, j\}$, such that there is a cycle $S$ in $G$ with $i, j \in S$. Let $\hat{c}$ be the information graph game with information graph $\hat{G}=(N, \hat{E})$ and informed set $\hat{Z}$. Then $\hat{c}$ is concave and $A \operatorname{Core}(\hat{c})=A \operatorname{Core}(c)$.

The extreme elements of the anti-core of an information graph game can now be characterized as being precisely the marginal allocation vectors of its associated concave information graph game. It follows that the extreme elements of the anti-core of an information graph game are integer, since the marginal allocation vectors are integer. This observation is helpful in the proof of the following theorem.

Theorem 4.16 Let $(N, c)$ be an information game with information graph $G=(N, E)$ and informed set $Z$. Then the set of marginal allocation vectors of $c$ contains the set of extreme elements of the anti-core of $c$ as a subset.

Proof: Without loss of generality we assume that $G$ is a connected graph. Let $x$ be extreme in ACore $(c)$. We shall show that there exist coalitions $S_{1} \subseteq S_{2} \subseteq \ldots \subseteq S_{n}$ with the property $x\left(S_{k}\right)=c\left(S_{k}\right)$ and $\left|S_{k}\right|=k$ for $k=1,2, \ldots, n$. We shall provide a proof using an inductive argument. 
First we show that there exists a player $i \in N$ with $x_{i}=c(\{i\})$. Since $c(\{i\}) \leq 1$ for all $i \in N$, we have $x_{i} \leq 1$ for all $i \in N$. If $x_{i}=1$ for some $i \in N$, then apparently $i \notin Z$ and $x_{i}=1=c(\{i\})$. Therefore, suppose $x_{i}<1$ for all $i \in N$. From the integrality of $x$ it follows that $x_{i} \leq 0$ for all $i \in N$ and thus $x(N) \leq 0$. This can only be the case if $Z \neq \emptyset$ and $x_{i}=0$ for all $i \in N$. Thus, for an arbitrary player $i \in Z$ we have $x_{i}=0=c(\{i\})$.

Let $S \neq N$ be a coalition such that $x(S)=c(S)$. We shall prove that there exists a player $i \in N \backslash S$ such that $x(S \cup\{i\})=c(S \cup\{i\})$. Define for all $i \in N \backslash S$ the number $q_{i}$ as the number of components of $S$ that contain a player adjacent to player $i$ and that contain no player in $Z$. Furthermore, define $d_{i}=0$ if $i \in Z$ or if there exists a component of $S$ that contains both a player in $Z$ and a player adjacent to $i$. Otherwise $d_{i}=1$.

It is not hard to see that $c(S \cup\{i\})=c(S)+d_{i}-q_{i}$. Clearly, $x_{i} \leq d_{i}-q_{i}$ for all $i \in N \backslash S$. Suppose that $x_{i}<d_{i}-q_{i}$ for all $i \in N \backslash S$. It then follows from the integrality of $x$ that $x_{i} \leq d_{i}-q_{i}-1$ for all $i \in N \backslash S$. And thus, $x(N) \leq c(S)+d(N \backslash S)-q(N \backslash S)-|N \backslash S|$.

If $S \neq N$ then each component of $S$ has at least one adjacent player in $N \backslash S$. (Here we use the fact that $G$ is connected.) Thus, $q(N \backslash S) \geq c(S)$. Furthermore, notice that $d(N \backslash S)=|N \backslash S|$ if $Z=\emptyset$ and that $d(N \backslash S)<$ $|N \backslash S|$ if $Z \neq \emptyset$.

It follows that $x(N) \leq c(S)+d(N \backslash S)-q(N \backslash S)-|N \backslash S| \leq 0$ if $Z=\emptyset$ and that $x(N) \leq c(S)+d(N \backslash S)-q(N \backslash S)-|N \backslash S|<0$ if $Z \neq \emptyset$. In both cases we have $x(N)<c(N)$, a contradiction. We conclude that there is a player $i \in N \backslash S$ satisfying $x_{i}=d_{i} \quad q_{i}$, and consequently $x(S \cup\{i\})=c(S \cup\{i\})$.

\subsubsection{The anti-nucleolus of information graph games}

There is a very efficient way for computing the anti-nucleolus of an information graph game. In this subsection we shall provide a system of linear equations, with the property that the unique solution of this system coincides with the anti-nucleolus of the game.

Let $c$ be an information graph game with information graph $G$ and informed set, $Z$. We have seen in subsection 4.3.3 that the coalitions in $X$ are the only coalitions which are needed in the description of ACore $(c)$. The coalitions in $K$ are also the only coalitions we need to consider when we want to compute the anti-nucleolus of $c$. This will be shown in the sequel. Again, assume that the information graph $G$ is connected. 
Let $x \in \mathrm{AI}(c)$. The excess $e(S, x)$ of coalition $S \subseteq N$ at $x$ is defined by $e(S, x)=c(S)-x(S)$ and $\theta(x)$ is the vector whose coordinates are the excesses at $x$ arranged in non-decreasing order. Recall that the anti-nucleolus is defined as the vector which lexicographically maximizes the vector $\theta(x)$ over the anti-imputation set $\operatorname{AI}(c)$. When $\operatorname{ACore}(c) \neq \emptyset$ the anti-nucleolus is an element of $\mathrm{ACore}(c)$, so in this case one may restrict oneself to a maximization over $\mathrm{ACore}(c)$, implying that all excesses are non-negative.

Consider a coalition $S$, which is not connected in the information graph $G$ and let us denote its components with respect to $G$ by $S_{1}, \ldots, S_{k}$. Then $c(S)=\sum_{i=1}^{k} c\left(S_{k}\right)$. According to corollary 2.16 we do not have to consider coalition $S$ in the lexicographic maximization, so we only have to consider coalitions which are connected in $G$ in the lexicographic maximization.

Now consider a coalition $S$ which is connected in $G$. In section 4.3 .3 we obtained the equality (4.5)

$$
\sum_{K \in \mathcal{K}_{S}} x(K)=\left(\left|\mathcal{K}_{S}\right|-1\right) x(N)+x(S),
$$

for some suitably chosen collection $\mathcal{K}_{S} \subseteq \mathcal{K}$. Furthermore, we obtained the inequality (4.6)

$$
\sum_{K \in K_{S}} c(K) \leq\left(\left|K_{S}\right|-1\right) c(N)+c(S) .
$$

Combining this we find

$$
\sum_{K \in K_{S}} e(K, x) \leq e(S, x)
$$

for any efficient vector $x$.

Now, let $\bar{x}$ denote $\mathscr{A N}(K, c)$ and let $\bar{y}$ denote $A \mathcal{N}(\mathcal{K} \cup\{S\}, c)$. Since $\bar{x}, \bar{y} \in$ ACore $(c)$, it follows that all excesses $e(K, \bar{x})$ and $e(K, \bar{y})$ are nonnegative for all $K \in K_{S}$. Hence,

$$
e(K, \bar{x}) \leq e(S, \bar{x}) \text { for all } K \in K_{S}
$$

and

$$
e(K, \bar{y}) \leq e(S, \bar{y}) \text { for all } K \in \mathcal{K}_{S} .
$$

Apply theorem 2.15 to prove that $\bar{x}=\bar{y}$. Repeated application of theorem 2.15 learns us that $\mathscr{A N}(c)=\mathcal{A N}(K, c)$. 
Therefore, let us introduce the vector $\phi(x)$ whose coordinates are the excesses of the coalitions in $\mathcal{K}$, arranged in non-decreasing order. Computing the anti-nucleolus comes down to the lexicographic maximization of the vector $\phi(x)$.

The blocks of the information graph play an important role in the characterization of the anti-nucleolus by means of a system of linear equations. Therefore, we shall prove some properties of the blocks of a graph. Let $G=(N, E)$ be a connected graph and let $B$ be a block of $G$. For each $b \in B$ let $K_{B, b}$ denote the component of the graph $G_{b}$ which contains the set $B \backslash\{b\}$.

Lemma 4.17 Let $G=(N, E)$ be a connected graph and let $B \subseteq N$ be a block of $G$. Then the collection $\left(N \backslash K_{B, b}\right)_{b \in B}$ forms a partition of $N$.

Proof: Let $i \in N$. We have to prove that there is exactly one $\bar{b} \in B$ such that $i \in N \backslash K_{B \bar{b}}$. We first prove the existence of such a $\bar{b}$.

If $i \in B$, we choose $\bar{b}=i$. Then trivially $i \in N \backslash K_{B, \bar{b}}$. Now assume that $i \notin B$. Then choose $b \in B$ arbitrarily and let $p(i, b)=\left(i, v_{1}, \ldots, v_{k}, b\right)$ be an elementary path from $i$ to $b$. Let $\vec{b}$ be the first vertex of $B$ on this path. We shall prove that $i \notin K_{B, \bar{b}}$. To see this, let $q(i, b)=\left(i, w_{1}, \ldots, w_{l}, b\right)$ also be an elementary path from $i$ to $b$ a nd let $\hat{b}$ be the first vertex of $B$ on this path. Suppose $\bar{b} \neq \hat{b}$. Let $x$ be the last vertex that the paths $p(i, b)$ and $q(i, b)$ have in common before they enter the set $B$. Follow the path $p(i, b)$ backward from $\bar{b}$ to $x$ and the follow the path $q(i, b)$ forward from $x$ to $\hat{b}$. This way we have constructed an elementary path from $\bar{b}$ to $\hat{b}$ and besides its initial and final vertex this path uses only vertices outside $B$. Let $T$ denote the set of vertices on this path not including $\hat{b}$ and $\bar{b}$. Then $T \neq \emptyset$ since $x \in T$. Clearly, $B \cup T$ is a connected set. It is even 2 -connected. This is seen as follows. Choose $j \in B \cup T$. First assume $j \in T$. The removal of $j$ leaves the remaining vertices of $T \backslash\{j\}$ still connected to either $\bar{b}$ or $\hat{b}$. Hence, $B \cup T \backslash\{j\}$ is connected. Now assume $j \in B$. Since $B$ is 2-connected we have that $B \backslash\{j\}$ is connected. The vertices in $T$ are still connected to $B \backslash\{j\}$, since this set contains at least one of the vertices $\hat{b}$ and $\bar{b}$. Hence, $B \cup T \backslash\{j\}$ is connected. It follows that $B \cup T$ is 2-connected, contradicting the maximality of $B$. The reason for the contradiction was the assumption $\hat{b} \neq \bar{b}$. We conclude that $\hat{b}=\bar{b}$. It follows that every path from $i$ to $b$ contains the vertex $\bar{b}$. Equivalently, $i$ and $b$ lie in different components from the graph $G_{\overline{\mathrm{b}}}$. Hence, $i \notin K_{B, \bar{b}}$ or $i \in N \backslash K_{B, \bar{b}}$. 
To prove the uniqueness of $\bar{b}$ observe that we have constructed a path from $i$ to $\bar{b}$ that uses only vertices outside $B$. Hence, $i$ and $\bar{b}$ lie in the same component of the graph $G_{b}$ for all $b \in B \backslash\{\bar{b}\}$. It follows that $i \in K_{B, b}$ or $i \notin N \backslash K_{B, b}$ for all $b \in B \backslash\{\bar{b}\}$.

Lemma 4.18 Let $G=(N, E)$ be a connected graph and let $B$ denote the collection of blocks of $G$. Then we have $\sum_{B \in B}(|B|-1)=|N|-1$.

Proof: We shall give a proof using induction on the number of vertices of the graph. If $|\boldsymbol{N}|=1$, then there is one block which contains one element, so $\sum_{B \in B}|B|-1=0=|N|-1$. Assume that $|N|=n$ and that the lemma is true if $|N|<n$. First consider the case that the graph $G$ is 2-connected. Then there is one block of cardinality $n$, and it follows that the lemma is true for $n$ also. Now consider the case that $G$ is not 2-connected. Choose a vertex $v \in N$, such that the removal of $v$ causes the graph to break into at least 2 components. Let $V_{1}, V_{2}, \ldots, V_{k}$ denote the vertex sets of these components. For each $i \in\{1, \ldots, k\}$ let $G_{i}$ denote the subgraph of $G$ with vertex set $V_{i} \cup\{v\}$ and let $B_{i}$ denote the collection of blocks of the graph $G_{i}$. We shall prove that $B_{1}, \ldots, B_{k}$ forms a partition of $B$.

Let $B \in B$. Since any two adjacent vertices form a 2-connected set, it follows that $B$ contains at least two element3. Hence, $B \backslash\{v\}$ is a non-empty connected set. It follows that $B \backslash\{v\} \subseteq V_{i}$ for precisely one $\hat{\imath} \in\{1, \ldots, k\}$ and that $B$ forms a 2-connected set in the graph $G_{\hat{f}}$. If $B$ were not a maximal 2-connected set in $G_{\hat{i}}$, then $B$ would not be maximal in $G$ either, a contradiction. Hence, $B \in B_{\hat{i}}$. The uniqueness of $\hat{i}$ implies that $B \notin B_{i}$ for $i \neq \hat{i}$. So far, we have proved that each coalition in $B$ is a member of precisely one collection $B_{i}$. It remains to prove that $B \supseteq \cup_{i=1}^{k} B_{i}$.

Let $B \in B_{i} . B$ is also a 2-connected set in $G$. We have to prove that $B$ is maximal in $G$. Let $\bar{B} \in B$ be a block in the graph $G$ that contains $B$ as a subset. We have just proved that there is precisely one $\hat{\imath}$ such that $\bar{B}$ is a block in $G_{i}$. From the maximality of $B$ in $G_{i}$ it follows that $\hat{i}=i$ and $\bar{B}=B: B$ is a maximal 2-connected set in $G$.

According to the induction hypothesis we have $\sum_{B \in B_{i}}(|B|-1)=\left|V_{i}\right|$ for all $i \in\{1, \ldots, k\}$. It follows that

$$
\sum_{B \in B}(|B|-1)=\sum_{i=1}^{k} \sum_{B \in B_{i}}(|B|-1)=\sum_{i=1}^{k}\left|V_{i}\right|=|N|-1
$$


Let $K^{*}$ denote the collection of coalitions $K_{B, b}$ over all blocks $B$ and all $b \in B$. We trivially have $K^{*} \subseteq K$.

Lemma $4.19 K^{*}=K$.

Proof: It suffices to prove that $K \subseteq \mathcal{K}^{*}$, so let $K \in \mathcal{K}$. Choose $i \in N$ such that $K$ is a component of the graph $G_{i}$. Choose $j \in K$ such that $j$ is adjacent to $i$ in $G$ and choose a block $B$ which contains both $i$ and $j$. Such a block exists, since $\{i, j\}$ is 2-connected. $K_{B, i}$ is the component of the graph $G_{i}$ which contains $B \backslash\{i\}$. We conclude that $j \in K_{B, i}$. Since we also have $j \in K$, it follows that $K=K_{B, i}$ and hence, $K \in K^{*}$.

For each block $B$ of the (connected) information graph $G=(N, E)$ we define

$$
\Delta(B)=\sum_{b \in B} c\left(K_{B, b}\right)-(|B|-1) c(N) .
$$

Let $B$ denote the collection of all blocks of $G$ and consider the following system of equations.

$$
\left\{\begin{array}{l}
x\left(K_{B, b}\right)=c\left(K_{B, b}\right)-\Delta(B) /|B| \text { for all } B \in B \text { and for all } b \in B \\
x(N)=c(N)
\end{array}\right.
$$

Lemma 4.20 System (4.7) has exactly one solution.

Proof: Let us give each element of $K^{*}$ an index, say $K^{*}=\left\{K_{1}, \ldots, K_{m}\right\}$. Furthermore, let $A$ be the $(0,1)$-matrix whose $i$-th row is the incidence vector $1_{k_{i}}$, and whose $(m+1)$-th row consists solely of ones. Then system (4.7) is a system of the type $A x=b$.

In section 4.3.3 it was proved that

$$
\operatorname{ACore}(c)=\left\{x \in \mathbb{R}^{N} \mid x(K) \leq c(K) \text { for } K \in K \text { and } x(N)=c(N)\right\} .
$$

Since ACore $(c)$ has at least one extreme element, it follows that the collection of incidence vectors $\left\{1_{K} \mid K \in \mathcal{K}\right\}$ together with the all-one vector contains a set of $n$ independent vectors. According to lemma 4.19 each incidence vector $1_{K}(K \in K)$ is a row of the matrix $A$, and hence $A$ has rank $n$. Therefore, any system of the type $A x=b$, like system (4.7), can have at most one solution. However, the matrix $A$ is not square, so we cannot conclude yet 
that the system indeed has a solution. We shall prove that some of the restrictions are redundant.

Let $B \in B$ be a block of the graph $G$, and let $\bar{b} \in B$. We shall prove that the restriction

$$
x\left(K_{B, \bar{b}}\right)=c\left(K_{B, \bar{b}}\right)-\Delta(B) /|B|
$$

is implied by the restrictions corresponding to the other elements of the block $B$, together with the restriction $x(N)=c(N)$. According to lemma 4.17 the collection $\left(N \backslash K_{B, b}\right)_{b \in B}$ forms a partition of $N$. This implies that

$$
\sum_{b \in B} x\left(K_{B, b}\right)=(|B|-1) x(N) .
$$

Now it follows that

$$
\begin{aligned}
x\left(K_{B, \bar{b}}\right) & =(|B|-1) x(N)-\sum_{b \in B \backslash\{\bar{b}\}} x\left(K_{B, b}\right) \\
& =(|B|-1) c(N)-\sum_{b \in B \backslash\{\bar{b}\}} c\left(K_{B, b}\right)+(|B|-1) \Delta(B) /|B| \\
& =c\left(K_{B, \bar{b}}\right)-\Delta(B) /|B| .
\end{aligned}
$$

Thus, we can delete this restriction without changing the set of solutions of system (4.7). The same can be done for each block of $G$, so in total $|B|$ restrictions can be deleted. The number of restrictions that remains is

$$
1+\sum_{B \in B}(|B|-1)
$$

According to lemma 4.18 this number equals $n$. Hence, system (4.7) is equivalent to a system of the type $\tilde{A} x=\tilde{b}$, where $\tilde{A}$ is a square non-singular matrix. This system has exactly one solution.

Theorem 4.21 The unique solution of system (4.7) is the anti-nucleolus of the information graph game $c$.

Proof: Let us number the blocks of $G$ such that

$$
\Delta\left(B_{1}\right) /\left|B_{1}\right| \leq \Delta\left(B_{2}\right) /\left|B_{2}\right| \leq \ldots \leq \Delta\left(B_{m}\right) /\left|B_{m}\right|,
$$

where $m$ is the number of blocks of $G$. For all $x \in \operatorname{AI}(c)$ we have

$$
\sum_{b \in B_{1}} e\left(K_{B_{1}, b}, x\right)=\Delta\left(B_{1}\right)
$$


Therefore, the smallest excess of a coalition in $K^{*}$ (the first coordinate of $\phi(x))$ will be at most $\Delta\left(B_{1}\right) /\left|B_{1}\right|$. Equality is only possible if the first $\left|B_{1}\right|$ coordinates of $\phi(x)$ are equal to $\Delta\left(B_{1}\right) /\left|B_{1}\right|$. The solution of system (4.7) indeed has its first $\left|B_{1}\right|$ excesses equal to $\Delta\left(B_{1}\right) /\left|B_{1}\right|$, so in the lexicographic maximization of $\phi(x)$ we may restrict ourselves to imputations which satisfy

$$
e\left(K_{B_{1}, b}, x\right)=\Delta\left(B_{1}\right) /\left|B_{1}\right| \text { for all } b \in B_{1} .
$$

Given this restriction we know that the $\left(1+\left|B_{1}\right|\right)$-th coordinate of $\phi(x)$ can be at most $\Delta\left(B_{2}\right) /\left|B_{2}\right|$. Again, equality is only possible if the coordinates of $\phi(x)$, numbered $1+\left|B_{1}\right|$ through $\left|B_{1}\right|+\left|B_{2}\right|$, are equal to $\Delta\left(B_{2}\right) /\left|B_{2}\right|$. Since the solution of system (4.7) satisfies this property, we now see that we may restrict ourselves to imputations which satisfy

$$
e\left(K_{B_{2}, b}, x\right)=\Delta\left(B_{2}\right) /\left|B_{2}\right| \text { for all } b \in B_{2} .
$$

We can repeat this reasoning for the blocks $B_{3}, \ldots, B_{m}$, and we realize that we may restrict ourselves to imputations which satisfy

$$
e\left(K_{B, b}, x\right)=\Delta(B) /|B| \text { for all } B \in B \text { and } b \in B \text {. }
$$

Hence, the solution of system (4.7) gives us the anti-nucleolus of $c$.

\subsection{Minimum cost forest games}

In this section we shall deal with the situation in which there are several suppliers, each supplier offering a different type of service. Let $N=\{1, \ldots, n\}$ denote the set of customers as usual, and let $M=\{n+1, \ldots, n+m\}$ denote the set of suppliers. For each customer $i \in N$ let $M(i) \subseteq M$ denote the set of suppliers to which customer $i$ wishes to be connected. We shall assume throughout that $M(i) \neq \emptyset$ for all $i \in N$. Furthermore, let $d$ be a non-negative weight function on the edges in the complete graph with vertex set $N \cup M$. Suppose the coalition $S \subseteq N$ forms. The set of links $E$ that have to be established in order to connect each player $i \in S$ to $M(i)$ does not necessarily form a connected graph. In contrast to the model of ordinary MCST games with only one supplier, we allow the coalition $S$ to use any edge in the network to establish a link. Hence, the cost $c(S)$ that this coalition incurs is equal to the minimal cost of any forest that connects each player $i \in S$ to the set of suppliers $M(i)$. Games defined in this way 
are called minimum cost forest games, or briefly, MCF games. A MCF game with only one supplier is a monotonic MCST game. Hence, the class of MCF games contains the class of monotonic MCST games as a subclass.

Several other generalizations of MCST games or monotonic MCST games have been studied in literature. Granot and Granot[22] introduced fixed cost spanning forest games. In this model, like in our model, there may be more than one supplier, but in contrast to our model, all suppliers offer the same type of service, so the customers need to be connected to only one supplier. These games may have an empty anti-core. However, spanning forest games need not be defined on a complete graph and if the game is defined on a tree, then the anti-core is non-empty.

Another generalization of monotone MCST games, called spanning network games, was considered by van den Nouweland[40] and by van den Nouweland, Maschler and Tijs[42]. They showed that this class coincides with the class of monotone games.

Capacitated network design games were introduced by Skorin-Kapov and Beltran[55]. Their model unifies capacitated versions of fixed cost spanning forest games, location garnes and Steiner tree games, which we will discuss next.

Steiner tree games were introduced by Megiddo[37]. In this model we have only one supplier. However, a link not necessarily connects two customers or a customer and the central supplier, but also other nodes, called switches, can be used as the endpoint of a link if this is favourable. Furthermore, a coalition $S$ is also allowed to establish links with an endpoint in $N \backslash S$. The class of Steiner tree games is easily be seen to be a subclass of MCF games, by interpreting the switches as suppliers to which no customer needs to be connected. Steiner tree games were also studied by Skorin-Kapov[54]. In that paper a sufficient condition is given to guarantee the non-emptiness of the anti-core. Moreover, an efficient algorithm is given which constructs an element in the anti-core if the Steiner tree game satisfies this condition. Megiddo[37] observed that a Steiner tree game without any switches is a monotonic MCST game, and that the anti-core is non-empty in this case. However, if there are switches, then a Steiner tree game may have an empty anti-core. Megiddo gave a 5-player example in which he showed that this negative result even holds if the Steiner tree game is situated in the Euclidean plane.

In this section we are interested in sufficient conditions such that a MCF game has a non-empty anti-core. Megiddo's result shows that a MCF game has a non-empty anti-core if there is only one supplier and all the customers 
wish to be connected to this supplier. In the following we shall prove a generalization of this result. We first need some preliminary results. The following lemma is due to Aarts and Driessen[2].

Lemraa 4.22 Let $V$ be a finite set and let $d$ be a non-negative weight function on $V \times V$. Let $\Gamma$ be a spanning tree with node set $V$. For each pair $v, w \in V$, let $d_{v w}^{\Gamma}$ denote the maximum over all weights among the edges along the unique path from $v$ to $w$ in $\Gamma$. Then the following statements are equivalent.

i) $\Gamma$ is a minimal spanning tree in the network $(V, d)$.

ii) $d_{v w} \geq d_{v w}^{\Gamma}$ for each pair $v, w \in V$.

Proof: Suppose that i) holds. Choose $v, w \in V$ arbitrarily. Let $\{i, j\}$ be an edge of maximal weight on the path from $v$ to $w$ in $I$, so $d_{i j} \cdots d_{v w}^{\Gamma}$. Remove the edge $\{i, j\}$ from $\Gamma$ and insert the edge $\{v, w\}$. The resulting graph is again a spanning tree on $V$. From the minimality of $\Gamma$ it follows immediately that $d_{v w} \geq d_{v w}^{\Gamma}$. Hence, i) $\Rightarrow$ ii).

Suppose that ii) holds and let $T$ be a minimal spanning tree with respect to the weight function $d$. Let $k$ be the number of edges that $T$ and $\Gamma$ have in common. In case $k=|V|-1$, the proof is finished: $T$ and $\Gamma$ coincide, and it trivially follows that $\Gamma$ is a minimal spanning tree. Therefore, suppose that $k<|V|-1$. Choose $v, w \in V$, such that $\{v, w\}$ is an edge of $T$, but not of $\Gamma$. Now, remove the edge $\{v, w\}$ from $T$. Let us denote the resulting graph by $F$. $F$ consists of two components, where $v$ and $w$ lie in different components. Denote the vertex sets of these components by $K$ and $L(v \in K$ and $w \in L$ ).

Let $P_{v w}$ denote the set of edges along the path from $v$ to $w$ in the tree $\Gamma$. Obviously, at least one edge in $P_{v w}$ connects an element of $K$ with an element of $L$. Choose such an edge, say $\{k, l\}$. The edge $\{k, l\}$ does not belong to $T$, since $\{v, w\}$ is the only edge in $T$ which connects an element of $K$ with an element of $L$. Add $\{k, l\}$ to the forest $F$, and the result is a tree. Denote this tree by $\bar{T}$.

We have $d_{k l} \leq d_{v w}^{\Gamma} \leq d_{v w}$, where the first inequality follows from the definition of $d_{v w}^{\Gamma}$ and the second inequality is simply property ii). Hence, the sum of the weights in $\bar{T}$ is at most the sum of the weights in $T$. It follows that $\bar{T}$ is a minimal spanning tree, which has $k+1$ edges in common with $\Gamma$. The process of replacing edges can be proceeded to produce minimal trees which have $k+2, k+3, \ldots$ edges in common with $\Gamma$. We conclude that $\Gamma$ itself is a minimal tree. 
Suppose that $\Gamma$ is a minimal tree with respect to the weight function $d$. The following lemma expresses that the numbers $d_{v w}^{\Gamma}$ do not change when we compute them with respect to another minimal tree. This lemma is also due to Aarts and Driessen[2].

Lemma 4.23 Let $(V, d)$ be a network and let $\Gamma$ and $\Omega$ be two minimal spanning trees on $V$ in this network. For each pair $v, w \in V$, let $d_{v w}^{\Gamma}\left(d_{v w}^{\Omega}\right)$ denote the maximum over all weights among the edges along the unique path from $v$ to $w$ in the tree $\Gamma(\Omega)$. Then $d_{v w}^{\Gamma}=d_{v w}^{\Omega}$ for all $v, w \in V$.

Proof: Choose $v, w \in V$. Let $E^{\Gamma}$ denote the set of edges on the path from $v$ to $w$ in $\Gamma$ and let $E^{\Omega}$ denote the set of edges on the path from $v$ to $w$ in $\Omega$. Let $\{i, j\}$ be an edge of maximum weight in $E^{\mathrm{I}}$. Remove this edge from the tree $\Gamma$. The resulting graph is a forest consisting of two components, say $K$ and $L$, where $v \in K$ and $w \in L$. When we follow the path from $v$ to $w$ in $\Omega$, we must come across an edge $\{k, l\} \in E^{\Omega}$ with $k \in K$ and $l \in L$. Add $\{k, l\}$ to the forest, and the result is a tree again. From the definition of $d_{v w}^{n}$ it follows that $d_{v w}^{n} \geq d_{k i}$. From the minimality of $\Gamma$ it follows that $d_{k l} \geq d_{i j}$, while $\{i, j\}$ was chosen such that $d_{i j}=d_{v w}^{\Gamma}$. Combining this we conclude that $d_{v w}^{n} \geq d_{v w}^{r}$. Exchanging the roles of $\Gamma$ and $\Omega$, one proves in a similar way that $d_{v w}^{\Gamma} \geq d_{v w}^{n}$, and hence $d_{v w}^{\Gamma}=d_{v w}^{n}$.

Let $\Gamma$ be a minimal tree with respect to the weight function $d$. In the following we use the notation $\bar{d}_{v w}$ to denote the maximum weight of an edge along the path between $v$ and $w$ in $\Gamma$. Lemma 4.23 justifies this notation. We call the edge $\{v, w\}$ minimal if $\bar{d}_{v w}=d_{v w}$. We call the network $(V, d)$ minimal if $\bar{d}_{v w}=d_{v w}$ for all $v, w \in V$.

Lemma 4.24 Let $(V, d)$ be a network and let $\Gamma$ be a minimal spanning tree on $V$ in this network. Then

i) the cost of a minimal spanning tree on $V$ in the network $(V, \bar{d})$ equals the cost of a minimal spanning tree on $V$ in the original network $(V, d)$, and

ii) for each $U \subseteq V$ there exists a minimal spanning tree $\bar{\Gamma}$ on $V$ in the network $(V, \bar{d})$, such that $U$ is a connected set in $\bar{\Gamma}$.

Proof: It is trivial that $\overline{(\bar{d})}=\bar{d}$. Hence, the weight function $\bar{d}$ satisfies property ii) of lemma 4.22 . It follows that $\Gamma$ is also a minimal tree with 
respect to the weight function $\bar{d}$. Since $d_{v w}=\bar{d}_{v w}$ whenever $\{v, w\}$ is an edge in $\Gamma$, i) follows.

Let $U \subseteq V$. Suppose that there is no minimal tree with respect to the weight function $\vec{d}$, such that $U$ is connected in this tree. Then let $T$ be a minimal tree for which the number of components of $U$ is minimal, and let $U_{1}, \ldots, U_{k}$ denote its components $(k \geq 2)$. Pick two elements of $U$ that lie in different components, say $u$ and $v$. The path in $T$ from $u$ to $v$ contains at least one vertex outside $U$. Let $w$ be such a vertex. Let $\bar{u}$ be the last element of $U$ on the path from $u$ to $w$, and let $\bar{v}$ be the first element of $U$ on the path from $w$ to $v$. We have found $\bar{u}$ and $\bar{v}$ such that the path in $T$ from $\bar{u}$ to $\bar{v}$ contains solely vertices outside $U$, besides of course the vertices $\bar{u}$ and $\bar{v}$ themselves. According to lemma 4.23, $\bar{d}_{\overline{u v}}$ equals the maximum over all weights among the edges on the path from $\bar{u}$ to $\bar{v}$ in $T$. Remove the maximal edge on this path and insert the edge $\{\bar{u}, \bar{v}\}$. The result is a minimal tree in which $U$ consists of $k-1$ components, a contradiction. We conclude that there exists a minimal tree in which $U$ is connected.

Now we proceed with some results which characterize classes of MCF gannes with a non-empty anti-core.

Theorem 4.25 Let $c$ be an MCF game with player set $N$ and set of suppliers $M$, defined on the network $(N \cup M, d)$. Suppose that there is a player $i \in N$ with $M(i)=M$. Then the game has a non-empty anti-core.

Proof: Let $\hat{\imath}$ be such that $M(\hat{\imath})=M$. The optimal forest for the grand coalition must be a tree, since $\hat{i}$ has to be connected to all suppliers. Let us denote this tree by $\Gamma$. Let $\bar{c}$ be the MCF game associated with the minimal weight function $\bar{d}$. According to lemma 4.24 we have $\bar{c}(N)=c(N)$, and since $\bar{d}_{v w} \leq d_{v w}$ we have $\bar{c}(S) \leq c(S)$ for all $S \subseteq N$. Hence, ACore $(\bar{c}) \subseteq$ ACore $(c)$ and it will be sufficient to prove that $\mathrm{ACore}(\bar{c}) \neq \emptyset$.

According to lemma 4.24 it is possible to construct a minimal tree with respect to the weight function $\bar{d}$, such that $M \cup\{\hat{i}\}$ forms a connected set. Let $\bar{\Gamma}$ be such a tree. For each $i \in N \backslash\{\hat{z}\}$, define $f(i)$ as the vertex which is visited directly after $i$ on the path from $i$ to $\hat{i}$ in $\bar{\Gamma}$. Let $E=\{\{i, f(i)\} \mid i \in N \backslash\{\hat{i}\}\}$. Since the edges $\{i, f(i)\}$ are all different, the cardinality of $E$ equals $|N|-1$. Let $F$ denote the set of remaining edges of $\bar{\Gamma}$. Since $\bar{\Gamma}$ has $|N|+|M|-1$ edges, it follows that $|F|=|M|$. Furthermore, $E$ contains no edges with both endpoints in $M \cup\{\hat{i}\}$, so $F$ contains all of these edges. There are $|M|$ such edges, so it follows that $F$ consists of precisely the edges for which both endpoints lie in $M \cup\{\hat{i}\}$. 
Define the vector $x \in \mathbb{R}^{N}$ by $x_{i}=\bar{d}_{i, f(i)}$ for $i \in N \backslash\{\hat{i}\}$ and $x_{i}=$ $\sum_{\{v, w\} \in F} \bar{d}_{v w}$. In view of the fact that $E \cap F=\emptyset$ and that $E \cup F$ equals the edge set of $\bar{\Gamma}$, it follows that the vector $x$ satisfies $x(N)=\bar{c}(N)$.

Let $S \subseteq N$. We have to prove that $x(S) \leq \bar{c}(S)$. Suppose that $\hat{\mathbf{i}} \notin S$. We construct a graph with node set $N \cup M$ as follows. First, construct a minimal forest for coalition $S$, such that each player $i \in S$ is connected to his set of suppliers $M(i)$. Add the edges in $F$ to this graph together with the edges $\{i, f(i)\}$ for $i \in N \backslash(S \cup\{\hat{i}\})$ (only the ones which are not already in the forest). The resulting graph is connected. To see this, we show that for an arbitrary vertex $v \in N \cup M$ there exists a path from $v$ to $\hat{\text { if. For }}$ $v \in M \cup\{\hat{i}\}$ this is trivial, since the addition of the edge set $F$ to the graph ensures that $M \cup\{\hat{i}\}$ is connected. For each $i \in S$, the graph contains a path from $i$ to any element $m \in M(i)$. We have just observed that there exists a path from $m$ to $\hat{i}$. After concatenation of these two paths, we obtain a path from $i$ to $\hat{\text { in. For }} \boldsymbol{i} \in N \backslash(S \cup\{\hat{i}\})$, we follow the path $i, f(i), f(f(i)), \ldots$ until a vertex in $S \cup M \cup\{\hat{i}\}$ is reached. This will surely happen, since the edges in $E=\{\{i, f(i)\} \mid i \in N \backslash\{\hat{i}\}\}$ contain no cycle. Once a vertex in $S \cup M \cup\{\hat{i}\}$ is reached, it is trivial again that a path to $\hat{\mathrm{s}}$ exists. The construction costs of the graph are at most $\bar{c}(S)+x(N \backslash S)$. Since the graph is connected, it follows that $\bar{c}(S)+x(N \backslash S) \geq \bar{c}(N)=x(N)$, and thus, $x(S) \leq \bar{c}(S)$.

Now suppose that $\hat{\imath} \in S$. Again, we construct a graph with node set $N \cup M$. We do it in the following way. First, construct a minimal forest for coalition $S$. Then add the edges $\{i, f(i)\}$ for $i \in N \backslash S$ (only the ones which are not already in the forest). Also this graph is connected. To see this, we show that for an arbitrary vertex $v \in N \cup M$ there exists a path from $v$ to $\hat{\imath}$. Since $\hat{\boldsymbol{i}} \in S$, the optimal forest for $S$ must contain paths from $m$ to $\hat{i}$ for all $m \in M$. For $i \in S$, observe that the optimal forest for $S$ contains a path from $i$ to any $m \in M(i)$. Furtermore, there exists a path from $m$ to $\hat{i}$, so after concatenation of the two paths, we obtain a path from $i$ to $\hat{i}$. Finally, for $i \in N \backslash S$, follow the path $i, f(i), f(f(i)), \ldots$ until a vertex in $S \cup M \cup\{\hat{i}\}$ is reached, and from there it is trivial again that a path to $\hat{\imath}$ exists. The construction costs of the graph are at most $\bar{c}(S)+x(N \backslash S)$. Since the graph is connected, it follows that $\bar{c}(S)+x(N \backslash S) \geq \bar{c}(N)=x(N)$, and thus, $x(S) \leq \bar{c}(S)$.

We know that the anti-core is always non-empty if an MCF game has only one supplier, since then it is a monotone MCST game. The following example 
shows that the anti-core may be empty when there are three suppliers.

Example : Let $N=\{1,2,3\}$ be the set of players and let $M=$ $\{I, I I, I I I\}$ be the set of suppliers. Each player wishes to be connected to exactly one supplier: $M(1)=\{I\}, M(2)=\{I I\}$ and $M(3)=\{I I\}$. The weight of an edge between any two players is 3 , the weight of an edge between any two suppliers is 5. Furthermore, the weight of an edge between a player and the supplier to which he wishes to be connected is 5 , but the weight of an edge between him and to the two other suppliers is 3. See the graph in figure 4.4, where all edges of weight 3 are drawn. The cost of any two-player coalition is 9 . For example, the optimal forest for coalition $\{1,2\}$ consists of the edges $\{1, I I\},\{2, I\}$ and $\{1,2\}$, a total cost of 9 . However, $c(\{1,2,3\})=14$, since the grand coalition must use an edge of weight 5 . Suppose that the anti-core is not empty. Then for reasons of symmetry the allocation in which each player pays $c(N) / 3$ should be an element of the anti-core, but clearly it is not. Hence, the anti-core is empty.

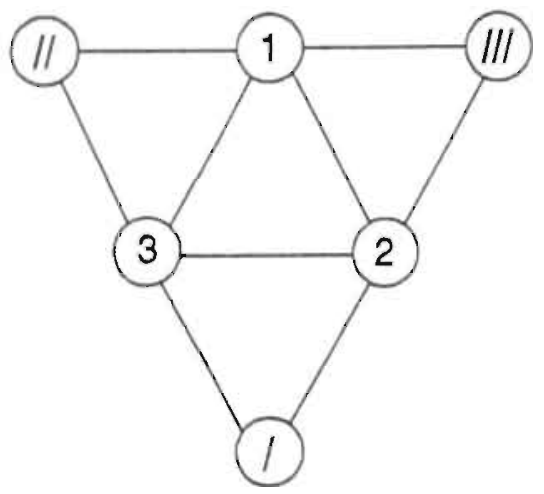

Figure 4.4

Theorem 4.25 can be regarded as a generalization of Granot and Huberman's theorem 4.1 on MCST garmes. The next result is another generalization of their theorem.

Theorem 4.26 Let $c$ be a MCF game with player set $N$ and set of suppliers $M$. Suppose that the optimal forest $\Gamma=(N \cup M, E)$ for the grand coalition consists of exactly $|M|$ components. Then $c$ has a non-empty anti-core. 
Proof : Clearly, each component of the forest $\Gamma$ contains exactly one supplier. For each $m \in M$ let $N(m) \subseteq N$ denote the set of players which are connected to supplier $m$. Since the players in $N(m)$ are not connected to any other supplier we conclude that $M(i)=\{m\}$ for all $i \in N(m)$. We shall prove that a tree-like vector lies in the anti-core of the game. For each $i \in N$ let $f(i)$ denote the vertex in $\Gamma$ which is visited directly after $i$ on the path from $i$ to its unique supplier in $M(i)$. Define the vector $x=\left(x_{1}, \ldots, x_{n}\right)$ by $x_{i}=d_{i, f(i)}$. It is trivial that $x(N)=c(N)$. It remains to prove that $x(S) \leq c(S)$ for all $S \subseteq N$. Let $S \subseteq N$, let $E_{S}$ be the edge set in an optimal forest for coalition $S$ and define $E_{N \backslash S}=\{\{i, f(i)\} \mid i \in N \backslash S\}$. Now, consider the graph with node set $N \cup M$ and edge set $E=E_{S} \cup E_{N \backslash S}$. The costs of construction of this graph are $x(N \backslash S)+c(S)$. Furthermore, one easily verifies that each player $i \in N$ is connected to his supplier $M(i)$ in this graph. Therefore $x(N \backslash S)+c(S) \geq c(N)$, and it follows that $x(S)=c(N)-x(N \backslash S) \leq c(S)$.

The following theorem treats the situation of two suppliers in which there is one supplier to which none of the customers needs to be connected. This supplier can be regarded as a switch, so that the MCF game is actually a Steiner tree game.

Theorem 4.27 Let $(N, c)$ be a Steiner tree game in which there is only one switch. Then $c$ has a non-empty anti-core.

Proof: Assume first that the grand coalition does not use the switch to connect all players to the supplier. In this case the core is non-empty according to theorem 4.26. So assume $N=\{1, \ldots, n\}$ uses the switch. Let, us denote the switch by $n+1$. Consider the MCST game $\bar{c}$ in which $n+1$ does not act as a switch but as an extra player, who also wishes to be connected to the supplier. Let $x \in \mathbb{R}^{n+1}$ denote a tree vector in this game. This vector trivially satisfies $x \geq 0$ and we have $x \in \operatorname{ACore}(\bar{c})$. According to theorem 4.5 there exists a vector $y \in \operatorname{Acore}(\bar{c})$ satisfying $y_{i} \geq 0$ for all $i \in N$ and $y_{n+1}=\bar{c}(N \cup\{n+1\})-\bar{c}(N)$. Observe that $y_{n+1} \leq 0$, since otherwise the grand coalition in the Steiner tree game $c$ would not have used the switch. If $x_{n+1}=y_{n+1}=0$, then set $z=x$. Otherwise we have $x_{n+1}>y_{n+1}$ and we set $\lambda=x_{n+1} /\left(x_{n+1}-y_{n+1}\right)$ and $z=(1-\lambda) x+\lambda y$. Now $z$ is an element in ACore $(\bar{c})$ satisfying $z \geq 0$ and $z_{n+1}=0$.

Let $S \subseteq N$. We have

$$
c(S)=\min \{\bar{c}(T) \mid S \subseteq T \subseteq N \cup\{n+1\}\} .
$$


Let $\bar{T}$ be such that $S \subseteq \bar{T} \subseteq N \cup\{n+1\}$ and $c(S)=\bar{c}(\bar{T})$. Since $z \geq 0$, we have

$$
z(S) \leq z(\bar{T}) \leq \bar{c}(\bar{T})=c(S)
$$

Furthermore,

$$
z(N)=z(N \cup\{n+1\})=\bar{c}(N \cup\{n+1\})=c(N) .
$$

Hence, the restriction of $z$ to the player set $N$ is an element of ACore (c).

We will show that a MCF game with two suppliers has a non-empty anti-core. For the proof of this we need the following notion and lemma.

Let $(V, d)$ be a network and let $U \subseteq V$. Consider the weight function $d^{U}$ defined by

$$
d_{i j}^{U}=d_{i j} \text { when } i, j \in V \backslash U
$$

and

$$
d_{i U}^{U}=\min \left\{d_{i j} \mid j \in U\right\} \text { when } i \in V \backslash U \text {. }
$$

Since the set $U$ acts as a single node in the network $\left(V \backslash U \cup\{U\}, d^{U}\right)$, we say that the set $U$ is contracted.

Lemma 4.28 Let $(V, d)$ be a network which is minimal, let $U \subseteq V$ and let $\left(V \backslash U \cup\{U\}, d^{U}\right)$ be the network in which $U$ is contracted. For any $S \subseteq V$ let $k(S)$ denote the cost of a minimal Steiner tree for $S$ in the network $(V, d)$ and for any $S \subseteq V \backslash U$ let $k^{U}(S)$ denote the cost of a minimal Steiner tree for $S$ in the contracted network $\left(V \backslash U \cup\{U\}, d^{U}\right)$. Then $k(S) \geq k^{U}(S \backslash U)+k(S \cap U)$ for all $S \subseteq V$. Moreover, $k(V)=k^{U}(V \backslash U)+k(U)$.

Proof: Let $S \subseteq V$, let $\Gamma$ be a minimal Steiner tree for $S$ in the network $(V, d)$, such that $S \cap U$ is connected. That such a tree exists follows from the minimality of $d$ and lemma 4.24. Let $E$ be the set of edges with both endpoints in $S \cap U$ in this tree, and let $F$ be the set of edges with at least one endpoint in $S \backslash U$. So

$$
k(S)=\sum_{e \in E} d_{\epsilon}+\sum_{e \in F} d_{e}
$$

Since $S \cap U$ is connected in $\Gamma$, it follows that $(S \cap U, E)$ is a tree. Hence,

$$
\sum_{t \in E} d_{t} \geq k(S \cap U)
$$


We shall also construct a tree for $S \backslash U$ in the network ( $V \backslash U \cup\{U\}, d^{U}$ ). Let $\{i, j\} \in F$. At least one of the endpoints of this edge, say $i$, lies in $S \backslash U$. If also $j \in S \backslash U$, then use this edge in the tree. Otherwise, use the edge $\{i, U\}$ (with smaller weight than $\{i, j\}$ in the network $(V, d)$ ) instead. These edges together form a tree for $S \backslash U$ in the network $\left(V \backslash U \cup\{U\}, d^{U}\right)$. The total added weight is at most $\sum_{e \in F} d_{e}$. Hence,

$$
\sum_{e \in F} d_{e} \geq k^{U}(S \backslash U)
$$

We conclude that

$$
\boldsymbol{k}(S) \geq k(S \cap U)+k^{U}(S \backslash U) .
$$

To prove that $k(V)=k(U)+k^{U}(V \backslash U)$ let $\Gamma_{U}$ be a minimal Steiner tree for $U$ in the network $(V, d)$ and let $\Gamma_{V \backslash U}$ be a minimal Steiner tree for $V \backslash U$ in the network $\left(V \backslash U \cup\{U\}, d^{U}\right)$.

Let $E$ denote the edge set of $\Gamma_{U}$. Define the edge set $F$ as follows. If $\{i, j\}$ is an edge in $\Gamma_{V \backslash U}$ with $i \in V \backslash U$ and $j \in V \backslash U$, then put $\{i, j\} \in F$. If $\{i, U\}$ is an edge in $\Gamma_{V \backslash U}$, then find $j \in U$ such that $d_{i U}^{U}=d_{i j}$ and put $\{i, j\} \in F$. Now $(V, E \cup F)$ forms a tree with node set $V$ in the network $(V, d)$. Hence,

$$
k(V) \leq \sum_{e \in E} d_{e}+\sum_{e \in F} d_{e}=k(U)+k^{U}(V \backslash U) .
$$

The reversed inequality was already proved, so it follows that

$$
k(V)=k(U)+k^{U}(V \backslash U) .
$$

Theorem 4.29 Let $(N, c)$ be a $M C F$ game with set of suppliers $M$, defined on the network $(N \cup M, d)$. If $|M|=2$, then $c$ has a non-empty anti-core.

Proof: We denote the two suppliers by $b$ (black) and $w$ (white). Furthermore, we denote the set of players who wish to be connected to $b$ by $B$, and the set of players who wish to be connected to $w$ by $W$. We assume $B \cap W=\emptyset$, since otherwise the anti-core is non-empty according to theorem 4.25. Furthermore, we assume that all minimal forests for $N$ are spanning trees with node set $N \cup\{w\} \cup\{b\}$, since otherwise the anti-core is non-empty according to theorem 4.26 . 
First assume that the weight function $d$ is minimal. Let $\bar{B}=B \cup\{b\}$ and $\bar{W}=W \cup\{w\}$. Furthermore, let $(W, \bar{c})$ be the Steiner tree game in the network $\left(\bar{W} \cup\{\bar{B}\}, d^{\bar{B}}\right)$, where $W$ is the player set, $w$ is the supplier and $\bar{B}$ is contracted to one node, which acts as the only switch in this Steiner tree game. According to theorem 4.27 this game has a non-empty anti-core. Choose $x \in \operatorname{ACore}(\bar{c})$.

Let $\left(B, c_{B}\right)$ be the subgame of $(N, c)$ restricted to the player set $B$, i.e. $c_{B}(S)=c(S)$ for all $S \subseteq B$. Since the weight function $d$ is minimal, it follows from lemma 4.24 that for each $S \subseteq B$ there exists a minimal forest for $S$ in which $S \cup\{b\}$ is connected. Hence, this minimal forest is a minimal tree with node set $S \cup\{b\}$. We conclude that $\left(B, c_{B}\right)$ is a MCST game. Such a garne has a non-empty anti-core, so choose $y \in \operatorname{ACore}\left(c_{B}\right)$.

Let $S \subseteq N$. If the minimal forest for coalition $S$ consists of a minimal Steiner tree that connects the nodes of $(S \cap W) \cup\{w\}$ plus a minimal Steiner tree for $(S \cap B) \cup\{b\}$, then we have

$$
c(S)=c(S \cap W)+c(S \cap B) \geq \bar{c}(S \cap W)+c_{B}(S \cap B) .
$$

If the minimal forest for coalition $S$ consists of one Steiner tree that connects all nodes in $S \cup\{w\} \cup\{b\}$, then we have according to lemma 4.28 that

$$
\begin{aligned}
c(S) & =k(S \cup\{b\} \cup\{w\}) \\
& \geq k^{\bar{B}}((S \cap W) \cup\{w\})+k((S \cap B) \cup\{b\}) \\
& =\bar{c}(S \cap W)+c_{B}(S \cap B) .
\end{aligned}
$$

Furthermore, we have

$$
c(N)=\bar{c}(W)+c_{B}(B) .
$$

If we define $z=(x, y)$, then

$$
z(S)=y(S \cap B)+x(S \cap W) \leq c_{B}(S \cap B)+\bar{c}(S \cap W) \leq c(S)
$$

and

$$
z(N)=y(B)+x(W)=c_{B}(B)+\bar{c}(W)=c(N) .
$$

We conclude that $\operatorname{ACore}(c) \neq \emptyset$, which proves that the lemma is true when the network is minimal.

Now assume that $(N \cup M, d)$ is not minimal. Then choose an edge $\{i, j\}$ which is not minimal. Lower the weight of the edge $\{i, j\}$ until one of the following two happens.

i) The edge $\{i, j\}$ becomes minimal. 
ii) An optimal forest exists which consists of two components.

Let us denote the game with the lowered weight function by $\tilde{c}$. Since $\tilde{c} \leq c$ and $\tilde{c}(N)=c(N)$, it is sufficient to prove that $\operatorname{ACore}(\tilde{c}) \neq 0$. If ii) is the case, this follows from theorem 4.26. Otherwise, we choose another edge $\{i, j\}$ which is not minimal and we repeat the process. We continue until ii) happens or until the weight function becomes minimal. In both cases we conclude that the anti-core is non-empty. 



\section{Chapter 5}

\section{Assignment games}

\section{This chapter is based on Kuipers[30].}

\subsection{Assignment problems}

In this section we consider the multi-dimensional assignment problem. We derive upper bounds for the ratio between the value of its $L P$-relaxation and the value of the integer problem. Furthermore, the worst case behaviour of a greedy algorithm for the p-dimensional problem is analysed. In section 5.2 these results are used in the analysis of assignment games.

Consider the following situation. Given are a set $A$ of amplifiers, as set $B$ of pairs of loud-speakers and a set $C$ of cd-players. With these we are able to assemble a number of stereo-sets (at most $\min (|A|,|B|,|C|)$ ) consisting of one amplifier, one cd-player and one pair of loud-speakers. Not ail $c$ d-players, amplifiers and loud-speakers need to be identical and therefore some stereosets that can be assembled may have a higher appreciation than others. Let $k_{a b c}$ denote the appreciation given to the stereo-set consisting of amplifier $a \in A$, pair of loud-speakers $b \in B$ and cd-player $c \in C$. The problem is to assemble a number of stereo-sets such that the total added appreciation of these sets is maximized. This can be formulated as an integer programming problem.

$$
\text { IP } \quad \begin{array}{lll}
\max & \sum k_{a b c} x_{a b c} & \\
\text { subject to } & \sum_{b, c} \boldsymbol{x}_{a b c} \leq 1 & \forall a \in A \\
& \sum_{a, c}: \boldsymbol{x}_{a b c} \leq 1 & \forall_{b \in B} \\
& \sum_{a, b} \boldsymbol{x}_{a b c} \leq 1 & \forall_{c \in C} \\
& \forall_{a b c}: \boldsymbol{x}_{a b c} \in\{0,1\} &
\end{array}
$$


IP describes a 3-dimensional assignment problem. It is a maximization version in which the restrictions are inequalities, reflecting that it is not necessary to use every component in a stereo-set. So this version differs slightly from the standard formulation, in which the total weight is minimized, the restrictions are equalities and $|A|=|B|=|C|$. The standard version is well-known to be $N P$-hard. It is $N P$-hard even when all weights $k_{a b c}$ are 0 or 1 (see e.g. Garey and Johnson[20]). By subtracting 1 from all weights in such an instance, we obtain an instance where all weights are 0 or -1 . Hence, the standard version is also $N P$-hard when all weights are 0 or -1 . The maximization version with weights 0 and 1 is easily transformed into a minimization version with weights 0 and -1 by altering the signs of all weights. Hence, the two versions are equivalent when $|A|=|B|=|C|$. We conclude that also IP is $\mathcal{N} P$-hard. Furthermore, no $\epsilon$-approximate polynomial algorithm exists for the standard version unless $P=N P$ (see Crama and Spieksma[10]). For our version the case is different, as will become clear in the proof of theorem 5.1. From now on we only discuss the maximization version with inequality constraints.

By $N$ we denote $A \cup B \cup C$, the collection of all objects in the assignment problem. By $v_{I P}(N)$ we denote the value of an instance of the assignment problem. The $L P$-relaxation of $I P$ is obtained by replacing the constraints $x_{a b c} \in\{0,1\}$ by $x_{a b c} \geq 0$. We denote the value of the $L P$-relaxation by $v_{L P}(N)$.

An obvious generalization is possible to p-dimensional assignment problems. In the p-dimensional case it is the aim to construct an optimal set of p-tuples, where the $p$ objects of each tuple are to be chosen from the sets $A_{1}, \ldots, A_{p}$ respectively.

Suppose we have an instance of the 3-dimensional assignment problem in which $|A|=|B|=|C|=\mathbf{n}$ and in which all weights $k_{a b c}$ are non-negative. Consider the following greedy algorithm to solve the problem.

$$
\begin{aligned}
& \tilde{x}=0 ; i=0 ; A_{i}=A ; B_{i}=B ; C_{i}=C ; \\
& \text { while } i<n \\
& \underline{\text { begin }} \\
& \quad \text { find } a_{i} \in A_{i}, b_{i} \in B_{i}, c_{i} \in C_{i} \text { such that } k_{a, b i c} \text { is } \\
& \quad \text { maximal in }\left\{k_{a b c} \mid a \in A_{i}, b \in B_{i}, c \in C_{i}\right\} ; \\
& \quad A_{i+1}=A_{i} \backslash\left\{a_{i}\right\} ; B_{i+1}=B_{i} \backslash\left\{b_{i}\right\} ; C_{i+1}=C_{i} \backslash\left\{c_{i}\right\} ; \\
& \quad \tilde{x}_{a_{i} b_{i} c_{i}}=1 ; \\
& \quad i=i+1 ; \\
& \text { end }
\end{aligned}
$$


In case $A, B$ and $C$ do not have the same cardinality or in case some of the weights are negative, the greedy algorithm should stop when no triples are left or when only triples with negative weight are left. The algorithm terminates with a feasible solution of the assignment problem, although this solution need not be optimal. On the other hand the ratio between the value of the greedy solution and the value of an optimal solution is bounded, as is proved in the following theorem.

Theorem 5.1 For any instance of the p-dimensional assignment problem the greedy algorithm constructs a solution with value at least $\frac{1}{p} v_{L P}(N)$.

Proof: To avoid notational complexity we prove the theorem for $p=3$. The generalization to arbitrary $p$ is straightforward. The theorem is trivially true when $|A|=|B|=|C|=1$. Assume the theorem is true when $|A|=$ $|B|=|C|=n-1$. Let $(\bar{a}, \bar{b}, \bar{c})$ be the triple which is chosen in the first step of the greedy algorithm and let $N^{-}=N \backslash\{\bar{a}, \bar{b}, \bar{c}\}$. Furthermore, let $\operatorname{IP}(N)$ denote the value of the solution provided by the greedy algorithm. Let $x$ be an optimal LP-solution for $N$. Construct an LP-solution for $N^{-}$ by removing all triples associated with $\bar{a}, \bar{b}$ or $\bar{c}$. If we remove all weights associated with $\bar{a}$ we, we remove

$$
\sum_{b \in B, c \in C} k_{\bar{a} b c} x_{\bar{a} b c} \leq k_{\bar{a} \bar{b} \bar{c}} \sum_{b \in B, c \in C} x_{\bar{a} b c} \leq k_{\bar{a} \bar{b} \bar{c}} .
$$

The same upper bound holds if we remove the triples associated with $\bar{b}$ and $\bar{c}$, so in total at most $3 k_{\bar{a} \bar{b} \bar{c}}$ will be removed. We conclude that

$$
v_{L P}\left(N^{-}\right) \geq v_{L P}(N)-3 k_{\bar{a} \bar{b} \bar{c}} .
$$

Furthermore, it is clear that

$$
I P(N)=I P\left(N^{-}\right)+k_{\bar{a} \bar{b} \bar{c}} .
$$

Combining these results and using the induction hypothesis we find

$$
v_{L P}(N) \leq 3 \operatorname{IP}(N) .
$$

The theorem has two interesting consequences. First, it shows that the value of the $L P$-relaxation stays within $p$ times the value of the optimal 
integer solution. Second, since $v_{L P}(N) \geq v_{I P}(N)$, the greedy algorithm constructs an integer solution with value at least a fraction $\frac{1}{p}$ of the optimal integer solution. In a more general setting the second result was already proved by Hausmann, Korte and Jenkyns[25]. The following example shows that the greedy algorithm can actually reach its worst case bound of $\frac{1}{p}$ times the optirnal value. This was also proved by Hausmann et al.

Example: Define the $p \times p$ matrix $A$ as follows.

$$
A_{i j}= \begin{cases}i & \text { if } j<i \\ 1 & \text { if } j=i \\ i+1 & \text { if } j>i\end{cases}
$$

Define the p-row vector $b$ by $b_{j}=1$ for all $j$. We see that two different rows from the matrix $A$ have no equal entries at corresponding places and that row $i$ from the matrix $A$ always has its $i-t h$ entry equal to $b_{i}$.

Now, we construct a p-dimensional assignment problem as follows. By $P$ we denote the set $\{1,2, \ldots, p\}$. A p-tuple in $P^{p}$ has weight 1 when this tuple corresponds to the vector $b$ or to a row of the matrix $A$. Otherwise it has weight 0 . The value of this assignment problem is $p$, because all p-tuples that correspond to the rows in $A$ can be assigned simultaneously. However, the greedy algorithm may choose the tuple that corresponds to the vector $b$ in its first step and it will find a solution with value 1.

Let $A_{1}, \ldots, A_{p}$ denote the sets from which the p-tuples are to be constructed.

Lemma 5.2 Let $x$ be an extreme element of the feasible region of the LP. relaxation of the $p$-dimensional assignment problem. Suppose $\max _{i=1}^{p}\left|A_{i}\right|=$ $\left|A_{1}\right|$. Then $x$ has an $\bar{a} \in A_{1}$ that takes part in no more than $p-1$-tuples, i.e. there are at most $p-1 p$-tuples $\left(\bar{a}, a_{2}, \ldots, a_{p}\right)$ such that $x_{a_{1}, a_{2}, \ldots, a_{p}} \neq 0$.

Proof: The set of equations of the LP-relaxation of IP consists of $\prod\left|A_{i}\right|$ non-negativity constraints and $\sum\left|A_{i}\right|$ assignment constraints. The rows of the $\sum\left|A_{i}\right|$ assignment constraints are easily seen to be dependent. Therefore the row space of the assignment constraints has dimension at most $\sum\left|A_{i}\right|-1$. An extreme point of the polytope must then satisfy at least $\Pi\left|A_{i}\right|-\left(\sum\left|A_{i}\right|-1\right)$ of the non-negativity constraints with equality. Thus, an extreme point has at most $\sum\left|A_{i}\right|-1$ non-zeros. Now, suppose that all points $a \in A_{1}$ take part in at least $p$ different $p$-tuples, then this corresponds 
to a point in the polytope with at least $p\left|A_{1}\right|$ non-zeros, which is more than $\sum_{i=1}^{p}\left|A_{i}\right|-1$. The lemma follows.

We use lemma 5.2 to derive a sharper bound for the ratio $v_{L P}(N) / v_{I P}(N)$ in the following case.

Theorem 5.3 When all weights in a p-dimensional assignment problem are 0 or 1 , then $v_{L P}(N) \leq(p-1) v_{I P}(N)$.

Proof: Again, we prove the theorem for the case $p=3$. The theorem obviously holds when $|A|+|B|+|C| \leq 3$. Assume the theorem holds when $|A|+|B|+|C| \leq n$. Now, suppose $|A|+|B|+|C|=n+1$. Let $\hat{x}$ be an optimal solution of the $L P$-relaxation. We may assume that $|A|=\max (|A|,|B|,|C|)$ and that $\hat{x}$ is an extreme point. According to lemma 5.2 we know that there is an $\bar{a} \in A$ which takes part in at most 2 triples. When $B$ or $C$ is empty the theorem is trivial, so suppose $B$ and $C$ are non-empty. Choose $b_{1}, b_{2} \in B$ and $c_{1}, c_{2} \in C$ such that $\hat{x}_{\bar{a} b_{1} c_{1}}=\alpha_{1}, \hat{x}_{\bar{a} b_{2} c_{a}}=\alpha_{2}$ and $\hat{x}_{\bar{a} b c}=0$ in all other cases. We distinguish two possibilities.

i) $k_{\bar{a} b_{1} c_{1}}=k_{\bar{a} b_{2} c_{2}}=0$. Let $N^{-}$denote $N \backslash\{\bar{a}\}$. From the optimal LPsolution for $N$ we obtain a feasible solution for $N^{-}$with the same value by deleting the triples associated with $\bar{a}$. So we have $v_{L P}\left(N^{-}\right)=v_{L P}(N)$ and $v_{I P}\left(N^{-}\right) \leq v_{I P}(N)$. Using the induction assumption we find $v_{L P}(N)=$ $v_{L P}\left(N^{-}\right) \leq 2 v_{I P}\left(N^{-}\right) \leq 2 v_{I P}(N)$.

ii) At least one of the weights $k_{\bar{a} b_{i} c_{i}}$ equals 1 . Without loss of generality we assume that $\alpha_{1}$ is the largest of the $\alpha$ 's for which $k_{\bar{a} b_{i} c_{i}}=1$. Construct an $L P$-solution for $N^{-}=N \backslash\left\{\bar{a}, b_{1}, c_{1}\right\}$ by removing all triples associated with $\bar{a}, b_{1}$ and $c_{1}$. The total added weight that will be removed is at most $k_{\bar{a}_{b_{2} c_{2}}} \alpha_{2}+\alpha_{1}+2\left(1-\alpha_{1}\right) \leq 2$. Thus we have

$$
\begin{aligned}
& v_{L P}(N)-2 \leq v_{L P}\left(N^{-}\right) \\
& v_{I P}(N) \geq v_{I P}\left(N^{-}\right)+1
\end{aligned}
$$

Using the induction assumption we get $v_{L P}(N) \leq 2 v_{I P}(N)$.

It is well-known that for any instance of the 2-dimensional problem we have $v_{L P}(N)=v_{I P}(N)$. Hence, for the 2-dimensional case, the bound in theorem 5.3 holds for arbitrary weights. We conjecture that the theorem holds for arbitrary weights in the p-dimensional case with $p>2$ also. The following theorem states that the bound in theorem 5.3 is sharp in at least some cases. 
Theorem 5.4 When $p-1$ is a prime power there are instances of the $p$ dimensional assignment problem such that $v_{L P}(N)=(p-1) v_{I P}(N)$.

Proof: Let $p-1=q$ be a prime power and let $\mathbb{F}_{q}$ be a field with cardinality $q$. Addition in this field is denoted by ' $\Theta$ ', subtraction is denoted by ' $\theta$ ', and multiplication is denoted by ' $\because$ '.

Define for each $k$ in $\mathbb{F}_{q}$ the $q \times q$ matrix $A^{k}$ by

$$
A_{i j}^{k}=i \oplus k \cdot j
$$

Consider two rows in two different matrices, say row $i_{1}$ in matrix $A^{k_{1}}$ and row $i_{2}$ in matrix $A^{k_{2}}$. We ask the question whether these rows have an equal entry at corresponding places, say at place $j$. Therefore we solve the equation $i_{1} \oplus k_{1} \cdot j=i_{2} \oplus k_{2} \cdot j$. This equation has the unique solution $j=\left(k_{1} \ominus k_{2}\right)^{-1} \cdot\left(i_{2} \ominus i_{1}\right)$, where $a^{-1}$ denotes the unique inverse of a non-zero element $a \in \mathbb{F}_{q}$. It means that two arbitrary rows from different matrices $A^{k_{1}}$ and $A^{k_{2}}$ always have exactly one equal entry at corresponding places. The equation has no solution when $k_{1}=k_{2}$ and $i_{1} \neq i_{2}$, meaning that two different rows from one matrix do not have equal entries. Let $\underline{i}$ denote the $q$-column vector with all entries equal to $i$. Define

$$
B^{k}=\left[\underline{k} \mid A^{k}\right]
$$

We see immediately that any two rows from different matrices $B^{k}$ or two different rows from one matrix always have exactly one equal entry at corresponding places.

We construct a $(q+1)$-dimensional assignment problem as follows. By $\mathbb{I F}_{q}^{q+1}$ we denote the cartesian product of $q+1$ fields $\mathbb{F}_{q}$. A $(q+1)$-tuple in $\| F_{q}^{q+1}$ has weight 1 when this tuple corresponds to a row in one of the matrices $B^{k}$. Otherwise it has weight 0 . The value of this assignment problem is 1 , because it is impossible to choose more than 1 tuple with weight 1 . However, the $L P$-relaxation of the problem has value $q$, because the feasible solution in which all $q^{2}$ tuples with weight 1 are assigned for a fraction $\frac{1}{q}$ gives us value $q$ and theorem 5.3 says that we cannot do any better than this.

The construction in theorem 5.4 fails when $p-1$ is not a prime power, because in this case no finite field of cardinality $p-1$ exists. 


\subsection{Assignment games}

In a natural way the assignment problem gives rise to a cooperative game. Again, consider the situation at the beginning of section 5.1, but this time all amplifiers, loud-speakers and cd-players are in possession of individuals or players and each player possesses one object. Hence, we may identify players with objects, so we have a player set $N=A \cup B \cup C$. The worth $v_{I P}(S)$ of a coalition $S \subseteq N$ is the maximum value that can be achieved by forming combinations $(a, b, c)$ within $S$. L.e.

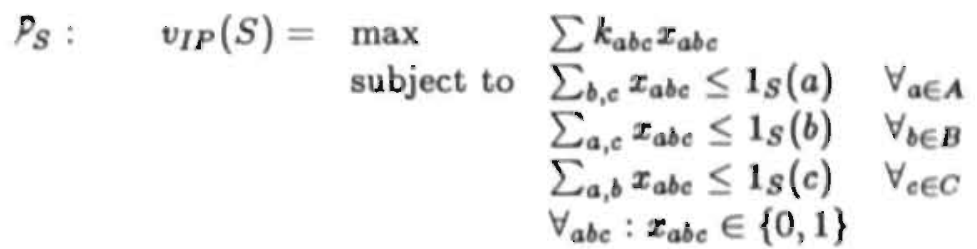

In this formulation the notation $1_{S}(x)$ stands for

$$
1_{S}(x)= \begin{cases}1 & \text { if } x \in S \\ 0 & \text { otherwise }\end{cases}
$$

By $v_{L P}(S)$ we denote the value of the $L P$-relaxation of problem $P_{S}$. We shall refer to the game $v_{I P}$ as the 3-dimensional assignment game. Of course a generalization to $p$-dimensional assignment games $(p \geq 2)$ is possible.

For $p=2$ we obtain the assignment game which was introduced by Shapley and Shubik[51], so the p-dimensional assignment game is a straightforward generalization of this. In their paper Shapley and Shubik prove that a 2-dimensional assignment game always has a non-empty core. Unfortunately, for $p \geq 3$ the core may be empty.

Example: Let $A=\{1,2\}, B=\{3,4\}$ and $C=\{5,6\}$. All weights of the triples in $A \times B \times C$ are 0 or 1 . The triples with weight 1 are $(1,3,5)$, $(1,4,6),(2,3,6)$ and $(2,4,5)$. Observe that this example was also used to show that the bound of 2 for the ratio between the LP-relaxation and integer version of a 3-dimensional assignment problem is sharp. We have $v_{I P}(N)=1$ and $v_{I P}(1,3,5)=v_{I P}(1,4,6)=v_{I P}(2,3,6)=v_{I P}(2,4,5)=1$. $A$ core element $x$ should satisfy $x(1,3,5) \geq 1, x(1,4,6) \geq 1, x(2,3,6) \geq 1$ and $x(2,4,5) \geq 1$. Adding these four inequalities and dividing by 2 we find $x(N) \geq 2$. But $x$ should also satisfy $x(N)=v_{I P}(N)=1$. We conclude that the core of $v_{I P}$ is empty. However, the game $v_{L P}$ has a non-empty core, since $(1 / 6,1 / 6,1 / 6,1 / 6,1 / 6,1 / 6)$ is an element of $i t$. 
For the reason that the core of a game may be empty, various solution concepts have been proposed that relax the core constraints somewhat. Probably the best known relaxation is the $\epsilon$-core, introduced by Shapley and Shubik[52]. The $\epsilon$-core of a game $v$ is defined by

$$
\operatorname{Core}_{\varepsilon}(v)=\left\{x \in \mathbb{R}^{n} \mid x(N)=v(N) \text { and } x(S) \geq v(S)-\epsilon \text { for all } S \subseteq N\right\} \text {. }
$$

Here we present another core-generalization in which we relax the condition that each coalition receives at least its own worth to the condition that each coalition receives at least a certain fraction of its own worth.

For a cooperative game $v$ we define its $k$-core as

$$
k \text {-Core }(v)=\left\{x \in \mathbb{R}^{n} \mid x(N)=v(N) \text { and } k x(S) \geq v(S) \text { for all } S \neq N\right\} .
$$

For obvious reasons we are interested in the minimal $k$ for which the $k$-core is non-empty. We define $k_{\min }(v)$ as the minimal $k$ for which $k$-Core $(v)$ is non-empty. We shall avoid pathological cases and assume throughout that $v(N)>0$, in which case the minimum exists. Elements of the $k_{\min }$-core have the following interpretation. Let $x$ be an element of the $k_{\min }$-core. Observe that $x$ is an efficient allocation such that each coalition gets at least $1 / k_{\text {min }}$ times its own worth. Furthermore, observe that for any efficient allocation there will always be a coalition that receives no more than $1 / k_{\min }$ times its own value. Thus, in this sense the allocation $x$ is the best one can achieve.

In case the game $v$ is zero-normalized the definition of the $k_{\min }$-core is equivalent to that of the least tax core, introduced by Tijs and Driessen in [59]. Since assignment games are zero-normalized games, all theorems concerning the $k_{\min }$-core are valid also for the least tax core.

Theorem 5.5 For a p-dimensional assignment game we have that $\boldsymbol{k}_{\min }=$ $v_{L P}(N) / v_{I P}(N)$.

Proof: Again we prove the theorem for the case $p=3$. Define $k^{*}=$ $v_{L P}(N) / v_{I P}(N)$. The dual of the $L P$-relaxation of $P_{S}$ is

$$
\begin{array}{ll}
Q_{S} \quad \min & \sum_{a \in A} u_{a} 1_{S}(a)+\sum_{b \in B} v_{b} 1_{S}(b)+\sum_{c \in C} w_{c} 1_{S}(c) \\
\text { subject to } & u_{a}+v_{b}+w_{c} \geq k_{a b c} \\
& u_{a} \geq 0 \\
& v_{b} \geq 0 \\
& w_{c} \geq 0
\end{array}
$$


Let $\left(u^{*}, v^{*}, w^{*}\right)$ be an optimal solution for $\mathcal{Q}_{N}$. Then for any $S=S_{A} \cup S_{B} \cup S_{C}$ with $S_{A} \subseteq A, S_{B} \subseteq B$ and $S_{C} \subseteq C$ we have

$$
\begin{array}{ll}
\sum_{a \in S_{A}} u_{a}^{*}+\sum_{b \in S_{B}} v_{b}^{*}+\sum_{c \in S_{C}} w_{c}^{*} & = \\
\sum_{a \in A} u_{a}^{*} 1_{S_{A}}(a)+\sum_{b \in B} v_{b}^{*} 1_{S_{B}}(b)+\sum_{c \in C} w_{c}^{*} 1_{S_{O}}(c) \geq \\
v_{L P}(S) \geq v_{I P}(S)
\end{array}
$$

and

$$
\begin{aligned}
& \sum_{a \in A} u_{a}^{*}+\sum_{b \in B} v_{b}^{*}+\sum_{c \in C} w_{c}^{*}= \\
& v_{L P}(N)=k^{*} v_{I P}(N)
\end{aligned}
$$

Hence $\frac{1}{k^{*}}\left(u^{*}, v^{*}, w^{*}\right) \in k^{*}-\operatorname{Core}\left(v_{I P}\right)$ and $k^{*} \geq k_{\min }$.

To prove that $k_{\min } \geq \boldsymbol{k}^{*}$ suppose that $\frac{1}{k_{\operatorname{mip}}}(u, v, w)$ is an element of $k_{\min }$ $\operatorname{Core}\left(v_{I P}\right)$. Then we have for each triple $(a, b, c) \in A \times B \times C$ that

$$
\begin{aligned}
& u_{a}+v_{b}+w_{c} \geq v_{I P}(\{a, b, c\})=k_{a b c} \\
& u_{a} \geq v_{I P}(\{a\})=0 \\
& v_{b} \geq v_{I P}(\{b\})=0 \\
& w_{c} \geq v_{I P}(\{c\})=0
\end{aligned}
$$

Thus $(u, v, w)$ is a feasible solution for $Q_{N}$, yielding that $k_{\min } v_{I P}(N)=$ $\sum u_{a}+\sum v_{b}+\sum w_{c} \geq v_{L P}(N)=k^{*} v_{I P}(N)$.

For any instance of a 2-dimensional assignment game we have $v_{L P}(N)=$ $v_{I P}(N)$. Applying theorem 5.5 we find that $k_{\min }=1$ and consequently the core is non-empty. So theorem 5.5 is a direct generalization of Shapley and Shubik's result. Shapley and Shubik not only proved that any solution of $\mathcal{Q}_{N}$ is a core-element, they also showed that the core of a 2-dimensional assignment game is precisely the set of solutions of $Q_{N}$. Completely analogous we have here that the core of $v_{L P}$ is precisely the set of solutions of $\mathcal{Q}_{N}$. In general however $\left\{x / k^{*} \mid x \in \operatorname{core}\left(v_{L P}\right)\right\}$ may be a proper subset of $k_{\min }-\operatorname{Core}\left(v_{I P}\right)$, hence not all solutions of $k_{\min }-\operatorname{Core}\left(v_{I P}\right)$ can be found by solving $\mathcal{Q}_{N}$.

Another generalization of 2-dimensional assignment games was considered by Curiel and Tijs[12]. Here they introduced multi-assignment games. Their model is very similar to ours, but not equivalent. In their model of a p-assignment games there are $p-1$ types of objects. The player set in a p-assignment game is divided into two types of players, buyers and sellers. Each seller possesses a bundle of $p-1$ objects, all of a different type. A transaction, where buyer $i$ buys an object of type 1 from seller $j_{1}$, an object of type 2 from seller $j_{2}$, etc. has value $k_{i, j_{1}, \ldots, j_{p-1}}$. Observe that one set 
of weights induces both a p-assignment game $v$ in the sense of Curiel and Tijs, and a p-dimensional assignment game $w$. Let $x$ be an element of the $k_{\min }(w)$-Core $(w)$. For a buyer $i$ in the game $v$ we define $y_{i}=x_{i}$. For a seller $j$, possessing the objects $j_{1}, \ldots, j_{p-1}$, we define $y_{j}=\sum_{h=1}^{p-1} x_{j_{h}}$. It is straightforward to verify that $y \in k_{\min }(w)$-Core $(v)$. Hence, we have the relationship $k_{\min }(v) \leq k_{\min }(w)$ for a $p$-assignment game $v$ and a $p$-dimensional assignment game $w$ defined on the same set of weights. This inequality may be strict.

In section 5.1 we derived some bounds for the ratio $v_{L P}(N) / v_{I P}(N)$. Using these results we conclude with some corollaries concerning the $k$-core of assignment games. These corollaries put bounds on the maximum value of $k_{\min }$ for an arbitrary assignment game.

Corollary 5.6 The p-core of a p-dimensional assignment game is never empty.

Proof: The corollary is a direct consequence of theorems 5.1 and 5.5.

Corollary 5.7 When all weights in a p-dimensional assignment game are 0 or 1 , then the $(p-1)$-core is non-empty.

Proof: The corollary is a direct consequence of theorems 5.3 and 5.5.

Corollary 5.8 When $p-1$ is a prime power the bound in corollary 5.7 is shar.p.

Proof: A direct consequence of theorems 5.4 and 5.5.

In analogy to the conjecture in section 5.1 it is conjectured that all $p$ dimensional assignment games have non-empty $(p \quad 1)$-core. Of course, for $p=2$ this conjecture is true. 


\section{Chapter 6}

\section{Extreme elements of the}

\section{core}

This chapter is based on Kuipers and Derks[31].

\subsection{Introduction}

In the previous chapters we paid considerable attention to the core (anticore) of various classes of games. In most cases we were satisfied when we were able to construct at least one core element. For MCST games we were able to do more than that, but despite the nice combinatorial structure of these games, we were still not able to characterize all extreme elements of the anti-core. In this chapter we are not so much interested in the characterization of individual core elements, but we shall provide sharp bounds on the number of extreme elements of the core and the B-restricted core.

Recall that for a game $v$ with player set $N$ and collection of formable coalitions $B$ the $B$-restricted core is defined as

$$
\operatorname{Core}(B, v)=\left\{x \in \mathbb{R}^{N} \mid x(N)=v(N) \text { and } x(S) \geq v(S) \text { for all } S \in B\right\} \text {. }
$$

We define the $B$-restricted upper set by

$$
\mathrm{U}(B, v)=\left\{x \in \mathbb{R}^{N} \mid x(S) \geq v(S) \text { for all } S \in B\right\} .
$$

In the following we shall provide bounds on the number of extreme elements of the upper set. When $N \in B$ then the restricted core is just a face of the restricted upper set. Hence, any bound we obtain for the number of extreme elements of the upper set is then also an upper bound for the core. 
For any two vectors $x, y \in \mathbb{R}^{n}$ their inner product is defined by $\sum_{i=1}^{n} x_{i} y_{i}$, and it is denuted by $x y$. For any element $x \in \mathrm{U}(B, v)$, let $B_{x} \subseteq B$ denote the collection of coalitions for which $x(S)=v(S) . x$ is an extreme element of $U(B, v)$ if and only if the incidence vectors corresponding to the coalitions in $B_{x}$ contain a set of $n$ linearly independent vectors. Hence, a trivial upper bound for the number of extreme elements of $U(B, v)$ is $\left(\begin{array}{c}|B| \\ n\end{array}\right)$. This bound is a gross overestimate. McMullen[35] showed that the polyhedron $P=\left\{x \in \mathbb{R}^{n} \mid A x \geq b\right\}$ has at most

$$
f(m, n)=\left(\begin{array}{c}
m-\left\lfloor\frac{n+1}{2}\right\rfloor \\
m-n
\end{array}\right)+\left(\begin{array}{c}
m-\left\lfloor\frac{n+2}{2}\right\rfloor \\
m-n
\end{array}\right)
$$

extreme elements, where $n$ is the number of rows of the matrix $A$ and $m$ is the number of columns. Furthermore, Gale[19] constructed examples of polyhedra having precisely $f(m, n)$ extreme elements, so that McMullen's bound cannot be further improved fcr arbitrary matrices $A$ (see also Chvatal[8] for these results). However, for the specific type of polyhedra we consider, an improvement of McMullen's bound is possible.

Let us define the polytope $Q^{B}$ as the convex hull of the origin 0 and the incidence vectors of all coalitions in $B$. We shall prove that the number of extreme elements of the upper set is bounded by $n$ ! times the $n$-dimensional volume of the polytope $Q^{B}$. Observe that $Q^{B}$ is contained in the unit hypercube, described by the restrictions $0 \leq x_{i} \leq 1$ for all $i \in\{1, \ldots, n\}$. Hence, $Q^{B}$ has a volume of at most 1 , and it follows that the upper set has at most $n$ ! extreme elements. However, if not every coalition $S \subseteq N$ is an element of $B$ we obtain a stronger bound.

Let $X \subseteq \mathbb{R}^{n}$ and $x \in X . x$ is said to be an interior point of $X$ if there exists $\epsilon>0$ such that for all $d \in \mathbb{R}^{n}$ with $\|d\| \leq 1$ and all $\delta$ with $0 \leq \delta \leq \epsilon$ we have $x+\delta d \in X$. Here $\|d\|$ denotes the Euclidian norm of the vector $d$. The set of all interior points of $X$ is called the interior of $X$.

Let $x$ be an extreme element of $\mathrm{U}(B, v)$ and let $Q_{x} \subseteq Q^{B}$ denote the convex hull of 0 and the incidence vectors of the elements of $B_{x}$. It is intuitively quite clear that $Q_{x} \cap Q_{y}$ has an empty interior for any two distinct extreme elements $x, y \in P$ (see also figure 6.1). We give a proof of this result in lemma 6.2. We need the following lemma as an intermediate result.

Lemma 6.1 Let $x_{1}, \ldots, x_{m_{2}} \in \mathbb{R}^{n}$ and let $X$ denote the convex hull of these points. If $z$ is an interior point of $X$ then there exist strictly positive $\lambda_{i}$, $(i=1, \ldots, m)$ with $\sum_{, i=1}^{m} \lambda_{i}=1$, such that $z=\sum_{i=1}^{m} \lambda_{i} x_{i}$. 

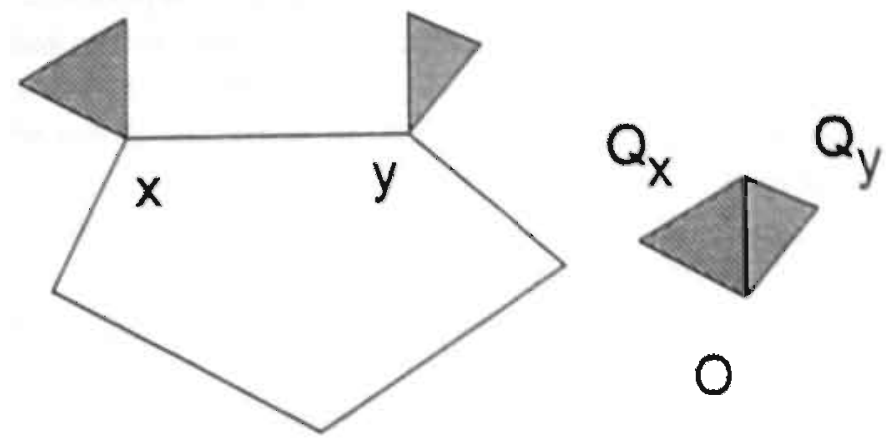

figure 6.1

Proof: Since $z$ lies in the interior of $X$, it is possible to choose $\epsilon>0$ such that $z+\epsilon \sum_{i=1}^{m}\left(z-x_{i}\right) \in X$. Hence, we can find $\mu_{i} \geq 0,(i=1, \ldots, m)$ with $\sum_{i=1}^{m} \mu_{i}=1$, such that $z+\epsilon \sum_{i=1}^{m}\left(z-x_{i}\right)=\sum_{i=1}^{m} \mu_{i} x_{i}$. It follows that $z=\sum_{i=1}^{m}\left(\mu_{i}+\epsilon\right) x_{i} /(1+\epsilon m)$. The lemma follows immediately by setting $\lambda_{i}=\left(\mu_{i}+\epsilon\right) /(1+\epsilon m)$.

Lemma 6.2 Let $x$ and $y$ be two distinct extreme elements of $U(B, v)$. Then $Q_{x} \cap Q_{y}$ has an empty interior.

Proof: Let $x$ and $y$ be two distinct extreme elements of $U(B, v)$ and suppose that $Q_{x} \cap Q_{y}$ has a non-empty interior. Choose $c$ in the interior of $Q_{x} \cap Q_{y}$. Obviously, $c$ lies also in the interior of $Q_{x}$. Therefore, it can be written as a convex combination of its extreme points, with all coefficients strictly positive, i.e. $c=\sum_{S \in B_{x}} \lambda_{S} 1_{S}$ with $\lambda_{S}>0$. We have that $y(\hat{S})>v(\hat{S})$ for at least one $\hat{S} \in B_{x}$. Since $\lambda_{\hat{S}}>0$ it follows that $c y=\sum_{S \in B_{x}} \lambda_{S} y(S)>\sum_{S \in B_{x}} \lambda_{S} v(S)=c x$. Analogously one proves that $c x>c y$, a contradiction.

As a consequence of lemma 6.2 , the $n$-dimensional volume of the union of polytopes $Q_{x}$ is simply the sum of their volumes. In the following we shall provide a lower bound on the volume of $Q_{x}$. This gives us then an upper bound on the number of polytopes $Q_{x}$ that can be contained in $Q^{B}$, or equivalently it gives us an upper bound on the number of extreme elements of $\mathrm{U}(B, v)$. 
A set of $n+1$ vectors $x_{0}, x_{1}, \ldots, x_{n} \in \mathbb{R}^{n}$ is called affinely independent if the vectors $x_{1}-x_{0}, \ldots, x_{n}-x_{0}$ are linearly independent. If the vectors $x_{0}, x_{1}, \ldots, x_{n}$ are affinely independent, then the convex hull of their endpoints is called a simplex.

Let $Q$ be a polytope. A collection of simplices $S$ is called a simplicial subdivision of $Q$ if

i) $\cup_{S \in S} S=Q$ and

ii) the interior of $S \cap T$ is empty for all $S, T \in S$ with $S \neq T$.

Let $\pi=\left(\pi_{1}, \ldots, \pi_{n}\right)$ be a permutation of $\{1, \ldots, n\}$. For each $k \in$ $\{1, \ldots, n\}$, let $u_{\pi, k} \in \mathbb{R}^{n}$ be the vector with ones at coordinates $\pi_{1}, \ldots, \pi_{k}$ and zeros elsewhere. The vectors $u_{\pi, 1}, \ldots, u_{\pi, n}$ are linearly independent, and hence the convex hull of the origin 0 and the endpoints of the vectors $u_{\pi, k}$ is a simplex. Let us denote this simplex by $S_{\pi}$. Furthermore, let us denote the collection of simplices $S_{\pi}$ over all permutations $\pi$ by $S$.

Lemma 6.3 The collection $S$ is a simplicial subdivision of the unit hypercube in $\mathbb{R}^{n}$.

Proof: Let $\pi$ and $\sigma$ be two different permutations of $\{1, \ldots, n\}$. We have to prove that $S_{\pi} \cap S_{\sigma}$ has an empty interior. Suppose $x$ lies in the interior of $S_{\pi} \cap S_{\sigma}$. Obviously, $x$ lies also in the interior of $S_{\pi}$. Therefore, we can write

$$
x=\sum_{i=1}^{n} \lambda_{i} u_{x_{i} i}
$$

with $\sum_{i=1}^{n} \lambda_{i}=1$ and $\lambda_{i}>0$ for all $i \in\{1, \ldots, n\}$. Observe that

$$
x_{\pi_{k}}=\sum_{i=1}^{k} \lambda_{i} .
$$

Now, using that $\lambda_{i}>0$ for all $i \in\{1, \ldots, n\}$ we find that

$$
x_{\pi_{1}}>x_{\pi_{2}}>\ldots>x_{\pi_{n}} .
$$

Analogously one proves that

$$
x_{\sigma_{1}}>x_{\sigma_{2}}>\ldots>x_{\sigma_{n}}
$$

a contradiction. 
We now have to prove that the union of elements in $S$ is the unit hypercube. Since each member of $S$ is trivially contained in the unit hypercube, it follows that also the union is contained in the unit hypercube.

Finally, to prove that the unit hypercube is contained in the union of elements of $S$, let $x$ be an element of the unit hypercube. Take a permutation $\pi$, such that $x_{\pi_{1}} \geq x_{\pi_{2}} \geq \ldots \geq x_{\pi_{n}}$. Then $x$ can be written as a nonnegative combination of the vectors $u_{\pi, i}$, say $x=\sum_{i=1}^{n} \lambda_{i} u_{\pi, i}$ with $\lambda_{i} \geq 0$ for all $i \in\{1, \ldots, n\}$. Observe that $u_{\pi, 1} u_{\pi, i}=1$ for all $i \in\{1, \ldots, n\}$. Furthermore, $u_{\pi, 1} x=x_{\pi_{1}} \leq 1$, since $x$ is an element of the unit hypercube. It follows that

$$
\sum_{i=1} \lambda_{i}=u_{\pi, 1} \sum_{i=1} \lambda_{i} u_{\pi, i}=u_{\pi, 1} x \leq 1 .
$$

Hence, $x$ is a convex combination of 0 and the vectors $u_{\pi, i}$ and it follows that $x \in S_{\pi}$.

The collection $S$ is well-known in literature (see e.g. Mara[32]). There an even stronger result is proved for $S$, namely that the intersection of any two distinct simplices in $S$ is a face of both simplices. A simplicial subdivision with this property is called a triangulation.

Let us denote the volume of an $n$-dimensional body $X \subseteq \mathbb{R}^{n}$ by $\mathcal{V}(X)$. The following theorem is well-known in linear algebra. For a proof we refer to Birkhoff and MacLane[6].

Theorem 6.4 Let $X \subseteq \mathbb{R}^{n}$ and let $A$ be a square matrix of dimension n. Then $\mathcal{V}(\{A x \mid x \in X\}=|\operatorname{det}(A)| \mathcal{V}(X)$, where $\operatorname{det}(A)$ denotes the determinant of the matrix $A$.

Using this theorem one easily proves the following lemma.

Lemma 6.5 Let $a_{1}, a_{2}, \ldots, a_{n} \in \mathbb{R}^{n}$ and let $A$ be the matrix with columns $a_{1}, a_{2}, \ldots, a_{n}$. Then the $n$-dimensional volume of the convex hull of the vectors $a_{1}, a_{2}, \ldots, a_{n}$ and 0 equals $|\operatorname{det}(A)| / n !$.

Proof: According to lemma 6.3, the simplices $S_{\pi} \in S$ form a simplicial subdivision of the unit hypercube. For reasons of symmetry all simplices $S_{\pi}$ have the same volume, and since there are $n$ ! of such simplices, each of them has volume $1 / n$ !. In particular, the simplex $S$, which is associated with the permutation $(1,2, \ldots, n)$ has volume $1 / n$ !. Let $B$ be the matrix with columns $a_{1}, a_{2}-a_{1}, a_{3}-a_{2}, \ldots, a_{n}-a_{n-1}$. Now, $\{B x \mid x \in S\}$ is precisely the simplex spanned by the vectors $a_{1}, \ldots, a_{n}$. Applying theorem 6.4 
we find that its volume equals $|\operatorname{det}(B)| \nu(S)=|\operatorname{det}(B)| / n !=|\operatorname{det}(A)| / n !$.

Now we are in a position to provide a lower bound on $\mathcal{V}\left(Q_{x}\right)$.

Lemma 6.6 Let $x$ be an extreme element of $U(B, v)$. Then $V\left(Q_{x}\right) \geq 1 / n !$.

Proof: Since $x$ is an extreme element of $\mathrm{U}(B, v)$, the incidence vectors in $\left\{1_{s} \mid S \in B_{x}\right\}$ contain a set of $n$ independent vectors, say $x_{1}, x_{2}, \ldots, x_{n}$. According to lemma 6.5 the volume of the convex hull of the points $x_{1}, x_{2}, \ldots, x_{n}$ and 0 equals $\left|\operatorname{det}\left(x_{1}, \ldots, x_{n}\right)\right| / n !$, where $\operatorname{det}\left(x_{1}, \ldots, x_{n}\right)$ denotes the determinant of the matrix with columns $x_{1}, \ldots, x_{n}$. All entries of this matrix are integer, so its determinant is also integer. The independency of the columns in the matrix ensures that the determinant is unequal to 0 and therefore, $\left|\operatorname{det}\left(x_{1}, \ldots, x_{n}\right)\right| \geq 1$. Consequently, the volume of the convex hull of the points $x_{1}, x_{2}, \ldots, x_{n}$ and 0 is at least $1 / n$ !. Since this convex hull is contained in $Q_{x}$, also $Q_{x}$ has a volume of at least $1 / n$ !.

Theorem 6.7 $U(B, v)$ has at most $n ! \mathcal{V}\left(Q^{B}\right)$ extreme elements.

Proof: Let $E$ denote the set of extreme elements of $\mathrm{U}(B, v)$. Clearly, $Q_{x} \subseteq Q^{B}$ for all $x \in E$. Hence, $\cup_{x \in E} Q_{x} \subseteq Q^{B}$ and

$$
\nu\left(Q^{B}\right) \geq V\left(\cup_{x \in E} Q_{x}\right)
$$

According to lemma, 6.2, the intersection of any two polytopes $Q_{x}$ and $Q_{y}$ with $x, y \in E(x \neq y)$ has an empty interior, and therefore

$$
\mathcal{V}\left(\cup_{x \in E} Q_{x}\right)=\sum_{x \in E} \mathcal{V}\left(Q_{x}\right)
$$

Furthermore, each polytope $Q_{x}$ has a volume of at least $1 / n$ !. Hence,

$$
\sum_{x \in E} \mathcal{V}\left(Q_{x}\right) \geq|E| / n !
$$

Cornbining these results the theorem follows.

Corollary 6.8 For any collection 8 and characteristic function $v$ the polyhedron $U(B, v)$ has at most $n$ ! extreme points. 
Proof: $Q^{B}$ is contained in the unit hypercube, described by the restrictions $0 \leq x_{i} \leq 1$ for all $i \in\{1, \ldots, n\}$. Hence, $\nu\left(Q^{8}\right) \leq 1$ and the corollary follows immediately from theorem 6.7.

In section 6.2 we shall prove that the bound mentioned in corollary 6.8 is sharp. Clearly, the maximum of $n$ ! extreme elements of $\mathrm{U}(B, v)$ can only be achieved if $B=2^{N}$. If $B$ is a proper subset of $2^{N}$, then $\nu\left(Q^{B}\right)$ is strictly less than 1 , and hence the bound in theorem 6.7 is strictly less than $n$ !. In section 6.2 we shall investigate structures on $B$, such that we can find nice expressions for the volume of the polytope $Q^{B}$.

\subsection{Graph induced polytopes}

Let $G$ be an undirected graph with vertex set $N=\{1, \ldots, n\}$. We say that a subset $S \subseteq N$ is $G$-connected if the subgraph on the vertices in $S$ is a connected graph. Let, $Q_{G}$ denote the convex hull of all incidence vectors of $G$-connected sets, including the null vector. $Q_{G}$ is called the $G$-induced polytope. In this section we shall be interested in determining an upper bound for the number of extreme elements of the polyhedron $\mathrm{U}(B, v)$ in case all elements of $B G$-connected.

According to theorem 6.7 it suffices to find an expression for the $n$ dimensional volume of the graph induced polytope $Q_{G}$ if we want to derive an upper bound for the number of extreme elements of $\mathrm{U}(B, v)$.

If $S$ is a simplicial subdivision of $Q_{G}$, then the volume of $Q_{G}$ is simply the sum of the volumes of the simplices in the simplicial subdivision. We have seen in lemma 6.3 that the volume of a simplex can easily be expressed in the determinant of a matrix. Hence, if we are able to construct a simplicial subdivision of the graph induced polytope, then this will enable us to find its volume.

Let $\pi=\left(\pi_{1}, \ldots, \pi_{s_{2}}\right)$ be: a permutation of $\{1, \ldots, n\}$. For each $k \in$ $\{1, \ldots, n\}$, let $u_{\pi, k}$ again be the vector with ones at coordinates $\pi_{1}, \ldots, \pi_{k}$ and zeros elsewhere, and let $U_{\pi, k}=\left\{\pi_{1}, \ldots, \pi_{k}\right\}$. Furthermore, let $V_{G, \pi, k}$ denote the $G$-component of $U_{\pi, k}$ which contains $\pi_{k}$. Finally, let $v_{G, \pi, i}$ be the $(0,1)$-vector with ones at places which correspond to elements in $V_{G, \pi, i}$ and zeros elsewhere. If no confusion can arise, we shall omit the subscript $G$ in our notation, so we write $V_{\boldsymbol{x}, k}$ and $v_{\boldsymbol{x}, \boldsymbol{k}}$.

Example: Consider the graph in figure 6.2, and let $\pi=(1,3,2,4)$. 
Then we have

$$
u_{\pi, 1}=\left(\begin{array}{l}
1 \\
0 \\
0 \\
0
\end{array}\right), u_{\pi, 2}=\left(\begin{array}{l}
1 \\
0 \\
1 \\
0
\end{array}\right), u_{\pi, 3}=\left(\begin{array}{l}
1 \\
1 \\
1 \\
0
\end{array}\right), u_{\pi, 4}=\left(\begin{array}{l}
1 \\
1 \\
1 \\
1
\end{array}\right)
$$

and

$$
v_{\pi, 1}=\left(\begin{array}{l}
1 \\
0 \\
0 \\
0
\end{array}\right), v_{\pi, 2}=\left(\begin{array}{l}
0 \\
0 \\
1 \\
0
\end{array}\right), v_{\pi, 3}=\left(\begin{array}{l}
1 \\
1 \\
1 \\
0
\end{array}\right), v_{\pi, 4}=\left(\begin{array}{l}
1 \\
1 \\
1 \\
1
\end{array}\right)
$$

In the example the vectors $v_{\pi, 1}, \ldots, v_{\pi, 4}$ are linearly independent. This is true in general and it will be proved in lemma 6.11. Therefore, the convex hull of the points $v_{\pi, 1}, \ldots, v_{\pi, n}$ and 0 is a simplex. Let us denote this simplex by $S_{G_{1 \pi}}$ and let us denote the collection of simplices $S_{G, \pi}$ over all permutations $\pi$ by $S_{G}$. The cardinality of $S_{G}$ can be less than $n !$, because two simplices $S_{G, \pi}$ and $S_{G, \sigma}$ can be the same, while the permutations $\pi$ and $\sigma$ are different.

Example: Consider the graph in figure 6.2 again, let $\pi=(1,3,2,4)$ and $\sigma=(3,1,2,4)$. The reader can easily verify that

$$
S_{G, \pi}=S_{G, G}=\operatorname{conv}\left\{\left(\begin{array}{l}
0 \\
0 \\
0 \\
0
\end{array}\right),\left(\begin{array}{l}
1 \\
0 \\
0 \\
0
\end{array}\right),\left(\begin{array}{l}
0 \\
0 \\
1 \\
0
\end{array}\right),\left(\begin{array}{l}
1 \\
1 \\
1 \\
0
\end{array}\right),\left(\begin{array}{l}
1 \\
1 \\
1 \\
1
\end{array}\right)\right\}
$$

The following lemmas give some insight in the structure of the collections $\left\{U_{\pi, 1}, \ldots, U_{\pi, n}\right\}$ and $\left\{V_{x, 1}, \ldots, V_{\pi, n}\right\}$.

Lemma 6.9 Let $G$ be an undirected graph with vertex set $N$, let $\pi$ be a permutation of $N$ and let $V_{x, 1}, \ldots, V_{x, n}$ be defined as above. For all $V, W \in$ $\left\{V_{\pi, 1}, \ldots, V_{\pi, n}\right\}$ at least one of the following three statements is true.
i) $V \subseteq W$,
ii) $W \subseteq V$, 


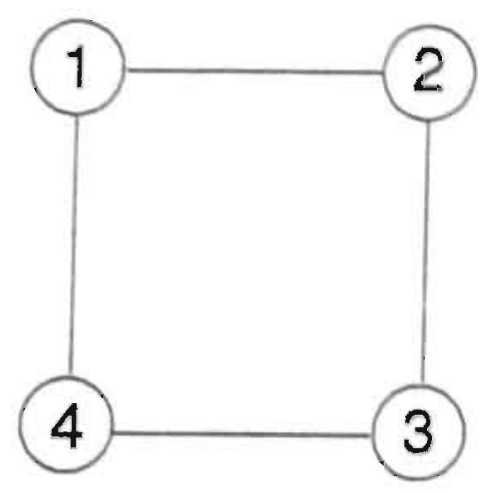

figure 6.2

iii) $V \cup W$ is not $G$-connected.

Proof: Suppose that neither $V \subseteq W$ nor $W \subseteq V$. Let $i, j$ be such that $V=V_{\pi, i}$ and $W=V_{\pi, j}$. Without loss of generality we assume that $i \leq j$. Then $V \cup W$ is a set which is strictly larger than $V_{\pi, j}$, it is contained in $U_{\pi, j}$ and it contains $\pi_{j}$. Then $V \cup W$ cannot be connected, since by definition $V_{\bar{\pi}, j}$ is the largest connected set which is contained in $U_{\pi, j}$ and which contains $\pi j$.

Lemma 6.10 Let $G$ be an undirected graph with vertex set $N$, let $\pi$ be a permutation of $N$ and let $U_{\pi, 1}, \ldots, U_{\pi, n}$ and $V_{\pi, 1}, \ldots, V_{\pi, n}$ be defined as above. Then

i) each component of $U_{\pi, k}$ is an element of $\left\{V_{\pi, 1}, \ldots, V_{\pi, k}\right\}$, and

ii) each component of $V_{\pi, k} \backslash\left\{\pi_{k}\right\}$ is an element of $\left\{V_{\pi, 1}, \ldots, V_{\pi, k-1}\right\}$.

Proof: Proof of i). We give a proof by induction on $k$. Let $S$ be a $G$ component of $U_{\pi, k}$. If $k=1$ then $S=U_{\pi, 1}=V_{\pi, 1}$ and it follows that the proposition is true in this case. Let $k>1$ be fixed and suppose that the proposition is true for $k-1$. If $\pi_{k} \in S$, then $S=V_{\pi, k}$ by definition and the proposition is proved for $k$ also. Now assume $\pi_{k} \notin S$. Then $S$ is also a component of $U_{x, k:-1}$ and according to the induction hypothesis, $S \in\left\{V_{\pi, 1}, \ldots, V_{\pi, k-1}\right\}$. This proves i). To see that ii) holds, it suffices to observe that each component of $V_{\pi, k} \backslash\left\{\pi_{k}\right\}$ is also a component of $U_{\pi, k-1}$. 
Let us now prove that the members of $S_{G}$ are indeed simplices. Moreover, their volume is exactly $1 / n$ !.

Lemma 6.11 Let $G$ be a graph with vertex set $N=\{1, \ldots, n\}$, let $\pi$ be a permutation of the elements of $N$, and let $S_{G, \pi}$ be defined as above. Then $S_{G, \pi}$ is a simplex and $\mathcal{V}\left(S_{G, \pi}\right)=1 / n !$.

Proof: As a consequence of lemma 6.10 we can write

$$
u_{\pi, k}=\sum_{i=1}^{k} \delta_{i k} v_{\pi, i}
$$

with $\delta_{i k} \in\{0,1\}$ and $\delta_{k k} \quad 1$. By permuting rows and columns, the matrix with columns $u_{\pi, 1}, \ldots, u_{\pi, n}$ can be transformed into an upper triangular matrix with ones on its diagonal. Hence.

$$
\left|\operatorname{det}\left(u_{\pi, 1}, \ldots, u_{\pi, n}\right)\right|=1 .
$$

It follows that

$$
\begin{aligned}
1 & =\left|\operatorname{det}\left(u_{\pi, 1}, \ldots, u_{\pi, n}\right)\right|= \\
& =\left|\operatorname{det}\left(\delta_{11} v_{\pi, 1}, \ldots, \sum_{i=1}^{n} \delta_{i n} v_{\pi, i}\right)\right|= \\
& =\left|\operatorname{det}\left(\delta_{11} v_{\pi, 1}, \ldots, \delta_{n n} v_{\pi, n}\right)\right|= \\
& =\left|\operatorname{det}\left(v_{\pi, 1}, \ldots, v_{\pi, n}\right)\right| .
\end{aligned}
$$

The fact that $\operatorname{det}\left(v_{\pi, 1}, \ldots, v_{\pi, n}\right) \neq 0$ proves that the vectors $v_{\pi, 1}, \ldots, v_{\pi, n}$ are linearly independent, and hence the convex hull of 0 and these vectors is a simplex. Furthermore, it follows from lemma 6.5 that

$$
V\left(S_{G, \pi}\right)=\left|\operatorname{det}\left(v_{\pi, 1}, \ldots, v_{\pi, n}\right)\right| / n !=1 / n !
$$

Lemma 6.12 Let $G$ be an undirected graph with vertex set $N$, let $\pi$ be a permutation of $N$ and let the simplices $S_{G, \pi}$ and $S_{G, a}$ be defined as usual. Exactly one of the following statements is true.

i) $S_{G, \pi}=S_{G, C}$,

ii) $S_{G, \pi} \cap S_{G, \sigma}$ has an empty interior. 
Proof: Suppose $S_{G, \pi} \neq S_{G, \sigma}$. Then at least one of the vectors $v_{\pi, i}$ differs from $v_{\sigma, j}$ for all $j \in\{1, \ldots, n\}$. Equivalently, there exists a $V \in$ $\left\{V_{\pi, 1}, \ldots, V_{\pi, n}\right\}$ such that $V \neq V_{\sigma, j}$ for all $j \in\{1, \ldots, n\}$. Let $X$ be the union of elements in $\left\{V_{\sigma, 1}, \ldots, V_{\sigma, n}\right\}$ that do not contain $V$ as a subset. We shall prove that $V \backslash X \neq \emptyset$. If $X \cap V=\emptyset$, this is trivial and we choose $v \in V \backslash X$. If this is not the case, we write $X=X_{1} \cup \ldots \cup X_{k}$ with $X_{\mathbf{i}} \in\left\{V_{\sigma, 1}, \ldots, V_{\sigma, n}\right\}$ and such that no two elements of $\left\{X_{1}, \ldots, X_{k}\right\}$ are contained in one another. Choose $X_{i}$ such that $X_{i} \cap V \neq \emptyset$. Since $V$ is not a subset of $X_{i}$, there exists a $v \in V \backslash X_{i}$ such that $v$ is adjacent to $X_{i}$. i.e $v$ is chosen such that $X_{i} \cup\{v\}$ is connected. If $v \in X_{j}$ for some $j \neq i$, then $X_{i} \cup X_{j}$ would be connected, contradicting lemma 6.9. Therefore $v \in V \backslash X$.

Observe that at least one element in $\left\{V_{\sigma, 1}, \ldots, V_{\sigma, n}\right\}$ contains $V$ as a subset, since otherwise $V \subseteq N=X$, contradicting the fact that $V \backslash X \neq \emptyset$. Let $Y$ be the intersection of all elements in $\left\{V_{\sigma, 1}, \ldots, V_{\sigma, n}\right\}$ that contain $V$ as a subset. According to lemma 6.9 these sets can be ordered inclusionwise. Therefore, $Y$ is just the smallest set in $\left\{V_{\sigma, 1}, \ldots, V_{\sigma, n}\right\}$, which contains $V$ as a subset. Since $Y \neq V$, it follows that $Y \backslash V \neq \emptyset$. Choose $w \in Y \backslash V$, such that $w$ is adjacent to $V$.

Now consider the hyperplane described by the restriction $x_{v}=x_{w}$. We shall prove that the simplex $S_{G, \pi}$ lies on one side of this hyperplane and that the simplex $S_{G, \sigma}$ lies on the other side. Define $p \in \mathbb{R}^{n}$ by

$$
p_{i}= \begin{cases}0 & \text { if } i \neq v, w \\ 1 & \text { if } i=v \\ -1 & \text { if } i=w\end{cases}
$$

Hence, $p$ is the normal of the hyperplane. Let $i \in\{1, \ldots, n\}$. First we shall prove that $p v_{\sigma, i} \leq 0$. If $V \subseteq V_{\sigma, i}$, then $V_{\sigma, i}$ contains both $v$ and $w$. Therefore $p v_{\sigma, i}=0$. If $V_{\sigma, i}$ does not contain $V$ as a subset, then it does not contain $v$. Therefore $p v_{\sigma, i} \leq 0$.

Now we shall prove that $p v_{x, i} \geq 0$. According to lemma 6.9, one of the following three statements is true. i) $V_{x, i} \subseteq V$. ii) $V \subseteq V_{x, i}$. iii) $V \cup V_{x, i}$ is not $G$-connected. If i) is the case then $V_{\pi, i}$ does not contain $w$. Therefore $p v_{\pi, i} \geq 0$. In case ii) we have that $v \in V_{\pi, i}$. It follows that $p v_{\pi, i} \geq 0$. Finally, in case iii), $V_{\pi, i}$ cannot contain $w$ because $V \cup\{w\}$ is $G$-connected. Therefore $p v_{\pi, i}=0$.

We conclude that a.l extreme elements of the simplex $S_{G_{i} \pi}$ lie on one side of the hyperplane and all extreme elements of the simplex $S_{G, a}$ lie on the other side. Hence, $S_{G, \pi} \cap S_{G, \sigma}$ has an empty interior. 
Lemma 6.12 suggests that $S_{G}$ is a good candidate for a simplicial subdivision of the graph induced polytope $Q_{G}$. Unfortunately, this is not always the case. Consider the graph in figure 6.2 again. The vector $\left(1, \frac{1}{2}, 1, \frac{1}{2}\right)$, being a convex combination of the $G$-connected vectors $(1,1,1,0)$ and $(1,0,1,1)$, is an element of $Q_{G}$. It is laborious, but not difficult to verify that this point lies in none of the simplices $S_{G, \pi}$. The reason is that the polytope $Q_{G}$ does not necessarily lie on one side of the hyperplane supported by the vectors $v_{x, 1}, \ldots, v_{x, n}$.

For a graph $G=(N, E)$ we denote the number of $G$-components of a set $S \subseteq N$ by $k_{G}(S)$ or simply by $k(S)$ if no confusion can arise. We call the graph $G$ component increasing if $k(S \backslash\{v\}) \leq k(T \backslash\{v\})$ for all $G$-connected sets $S$ and $T$ with $S \subseteq T$ and $v \in N$.

Lemma 6.13 Let $G=(N, E)$ be a component increasing graph and let $\pi$ be a permutation of $N$. Then the polytope $Q_{G}$ lies on one side of the hyperplane supported by the vectors $v_{\pi, 1}, \ldots, v_{\pi, n}$.

Proof: Define $p \in \mathbb{R}^{n}$ by $p_{\pi_{i}}=1-k\left(V_{\pi, i} \backslash\left\{\pi_{i}\right\}\right)$ for $i \in\{1, \ldots, n\}$. We shall prove by induction that $p v_{\pi, i}=1$ for all $i \in\{1, \ldots, n\}$. Geometrically, the vector $p$ is the normal of the hyperplane supported by the vectors $v_{\pi, 1}, \ldots, v_{\pi, n}$.

Clearly, $p v_{\pi, 1}=p_{\pi_{1}}=1$. Suppose $p v_{\pi, i}=1$ for $i \in\{1, \ldots, k-1\}$. Let us denote the unit vector, which has a one at cuordinate $\pi_{k}$ and zeros elsewhere, by $e_{\pi_{k}}$. In lemma 6.10 we proved that each component of $V_{\pi_{,} k} \backslash\left\{\pi_{k}\right\}$ is an element of the collection $\left\{V_{\pi, 1}, \ldots, V_{\pi, k-1}\right\}$. Consequently, we can write $v_{\pi, k}-e_{\pi_{k}}=\sum_{j=1}^{k-1} \delta_{j} v_{\pi, j}$ with $\delta_{j}$ equal to 0 or 1 for all $j$ and $\sum_{j-1}^{k-1} \delta_{j}=$ $k\left(V_{x, k} \backslash\left\{\pi_{k}\right\}\right)$. Using the induction hypothesis and the definition of $p$, it follows that

$$
p v_{\pi, k}=p \sum_{j=1}^{k-1} \delta_{j} v_{x, j}+p_{\pi_{k}}=\sum_{j=1}^{k-1} \delta_{j}+p_{\pi_{k}}=1 .
$$

Let $S \subseteq N$ and let $s$ denote its associated $(0,1)$-vector. We now show that $p s \leq k(S)$. We shall prove this by induction on the cardinality of $S$. Clearly, $p_{i} \leq 1$ for all $i \in\{1, \ldots, n\}$, so if $|S|=1$, the proposition is true. Suppose $|S|=m$ and that the proposition is true if the cardinality of $S$ is less than $m$. First assume that $S$ is $G$-connected. Let $k$ be such that $\pi_{k} \in S$ and that $\pi_{l} \notin S$ for $l>k$. Then $S \subseteq V_{\pi, k}$. Using the induction hypothesis, lemma 6.14 and the fact that $G$ is component increasing, we find

$$
p s \leq k\left(S \backslash\left\{\pi_{k}\right\}\right)+p_{\pi_{k}} \leq k\left(V_{\pi_{,} k} \backslash\left\{\pi_{k}\right\}\right)+p_{\pi_{k}}=1 .
$$


If $S$ has components $S_{1}, \ldots, S_{l}$, then the lemma follows from

$$
p s=\sum_{i=1}^{l} p s_{i} \leq l=k(S) .
$$

We conclude that $p x \leq 1$ for all $x \in Q_{G}$, and hence $Q_{G}$ lies on one side of the hyperplane $p x=1$, the hyperplane supported by the vectors $v_{\pi, 1}, \ldots, v_{\pi, n}$.

Component increasingness of a graph turns out to be equivalent to the property of cycle completeness.

Lemma 6.14 Let $G=(N, E)$ be an undirected graph. Then the following statements are equivalent.

i) $G$ is cycle complete.

ii) The collection of $G$-connected sets is closed under intersection.

iii) $G$ is component increasing.

Proof: i) $\Rightarrow$ ii): Let $S$ and $T$ be $G$-connected sets and suppose $v, w \in S \cap T$. Let $\left(s_{0}, \ldots, s_{n}\right)$ be a path from $v$ to $w$ which uses onily vertices in $S$ and let $\left(t_{0}, \ldots, t_{m}\right)$ be a path from $v$ to $w$ which uses only vertices in $T$ (so $s_{0}=t_{0}=v$ and $s_{n}=t_{m}=w$ ). Suppose $s_{i}=t_{i}$ for $i=1, \ldots, h$. If $h=n$ or $h=m$ then clearly we have a path from $v$ to $w$ which uses only vertices in $S \cap T$. Therefore, suppose $h<\min (n, m)$. Let $k$ be the smallest index greater than $h$, for which one of the $t_{i}$ 's equals $s_{k}$, say $t_{l}=s_{k}$. Such indices exist since $s_{n}=t_{m}=w$. Then $\left(s_{h}, \ldots, s_{k}\right)$ and $\left(t_{h}, \ldots, t_{l}\right)$ are two vertex-disjoint paths from $s_{h}=t_{h}$ to $s_{k}=t_{l}$. The set of vertices that lie on these two paths form a cycle. According to i), this set is complete, and therefore $\left\{s_{h}, s_{k}\right\}$ is an edge of $G$. Now, remove all vertices between $s_{h}$ and $s_{k}$ from the path $\left(s_{1}, \ldots, s_{n}\right)$ and remove all vertices between $t_{h}$ and $t_{l}$ from the path $\left(t_{1}, \ldots, t_{m}\right)$. Then both sequences remain paths from $v$ to $w$. After renumbering the remaining vertices in both paths we have $s_{i}=t_{i}$ for $i=1, \ldots, h+1$. Repeat this process until both paths are identical. The process ends with a path from $v$ to $w$, which uses only vertices in $S \cap T$. It follows that $S \cap T$ is connected.

ii) $\Rightarrow$ iii): Let $S$ and $T$ be $G$-connected sets with $S \subseteq T$ and $v \in S$. Suppose $T \backslash\{v\}$ has $k$ components. Denote these components by $T_{1}, \ldots, T_{k}$. Define $S_{i}=S \cap T_{i}$ for $i=1, \ldots, k$. According to ii), the sets $S_{i}$ are all 
connected. Furthermore, no union $S_{i} \cup S_{j}$ is connected, since otherwise $T_{i} \cup T_{j}$ would also be connected. Hence, the number of components of $S \backslash\{v\}$ is exactly the number of non-empty sets $S_{i}$, which is at most $k$.

$\neg$ i) $\Rightarrow \neg$ iii): Choose a cycle $v_{1}, \ldots, v_{n}, v_{1}$ which is not complete, say $\left\{v_{i}, v_{j}\right\} \notin E$. Assume that $i<j$ and that $j$ is the smallest index greater than $i$ such that $\left\{v_{i}, v_{j}\right\} \notin E$. Let $S=\left\{v_{i}, v_{j-1}, v_{j}\right\}$ and $T=\left\{v_{1}, \ldots, v_{n}\right\}$. Both $S$ and $T$ are $G$-connected and $S \subseteq T$. However, $S \backslash\left\{v_{j-1}\right\}$ consists of two components, while $T \backslash\left\{v_{j-1}\right\}$ is connected. Therefore, iii) does not hold.

As the reader suspects by now, cycle completeness of a graph turns out to be a sufficient condition to ensure that $S_{G}$ is a simplicial subdivision of $Q_{G}$. We conjecture that the condition is also necessary.

Theorem 6.15 Let $G$ be a cycle complete graph. Then the collection $S_{G}$ is a simplicial subdivision of $Q_{G}$.

Proof: We have already proved that the intersection of any two distinct elements of $S_{G}$ has an empty interior. Therefore, it suffices to prove that the union of elements of $S_{G}$ equals $Q_{G}$. Suppose $x \in Q_{G}$. Let $\pi$ be a permutation of $\{1, \ldots, n\}$ such that $x_{\pi_{1}} \geq x_{\pi_{2}} \geq \ldots \geq x_{\pi_{n}}$. We shall prove that $x \in S_{G, \pi}$.

Let $p$ denote the normal of the hyperplane supported by the vectors $v_{\pi, 1}, \ldots, v_{\pi, n}$. Since $G$ is component increasing, it follows from lemma 6.13 that $p x \leq 1$ and that $p v_{\pi, i}=1$ for all $i \in N$.

Observe that $x$ can be written as a non-negative combination of the vectors $u_{\pi, 1}, \ldots, u_{\pi, n}$, and since each $u_{\pi, i}$ can be written as a non-negative combination of the vectors $v_{\pi, 1}, \ldots, v_{\pi, n}$, it follows that $x$ can be written as a non-negative combination of the vectors $v_{\pi, 1}, \ldots, v_{\pi, n}$, say $x=\sum \lambda_{i} v_{\pi, i}$ with $\lambda_{i} \geq 0$ for all $i$. Using that $p v_{\pi, i}=1$ for all $i$, we find

$$
\sum \lambda_{i}=\sum \lambda_{i} p v_{\pi, i}=p x \leq 1
$$

In other words, $x$ is a convex combination of $v_{\pi, 1}, \ldots, v_{\pi, n}$ and 0 . We conclude that $x \in S_{G, \pi}$.

Let us define the permutation number of a graph $G$ as the cardinality of the collection $S_{G}$ and let us denote this number by $p_{G}$. Applying the previous results we obtain the following theorems. 
Theorem 6.16 Let $G$ be an undirected graph. The volume of the graph induced polytope $Q_{G}$ is at least $p_{G} / n !$. Equality holds if $G$ is a cycle complete graph.

Proof: The theorem is a direct consequence of lemma 6.11, lemma 6.12 and theorem 6.15.

Theorem 6.17 Let $(N, v)$ be a game and let $B \subseteq 2^{N}$ be a collection of coalitions such that each coalition in $B$ is connected with respect to a cycle complete graph $G=(N, E)$. Then the number of extreme elements of the polyhedron $U(B, v)$ is at most the permutation number $p_{G}$. In case $G$ is connected this bound also holds for Core $(B, v)$.

Proof: This is a direct consequence of theorem 6.7 and theorem 6.16.

The bound in theorem 6.17 is sharp, i.e. it is possible to construct examples for which the number of extreme elements of $U(B, v)$ is exactly $p_{G}$ in case $G$ is cycle complete. In the following we shall construct such an example.

A game $(N, v)$ is called convex if

$$
v(S)+v(T) \leq v(S \cup T)+v(S \cap T)
$$

for all $S, T \subseteq N$. Let us call a game strictly convex if

$$
v(S)+v(T)<v(S \cup T)+v(S \cap T)
$$

for all $S, T \subseteq N$ with $S \nsubseteq T$ and $T \nsubseteq S$. A strictly convex game $(N, v)$ satisfies

$$
v(S \cup\{i\})-v(S)<v(T \cup\{i\})-v(T)
$$

for all $i \in N$ and $S \subset T \subseteq N \backslash\{i\}$, where the symbol ' $C$ ' is used to denote a proper subset. This follows directly from (6.2), by letting the roles of $S$ and $T$ in (6.2) be played by $S \cup\{i\}$ and $T$.

The extreme elememts of the core of a convex game are precisely the $n !$ marginal allocation vectors. Some of these marginal allocation vectors may coincide, so that we cannot conclude yet that the core has $n$ ! extreme elements. Strict convexity of a game ensures that all marginal allocation vectors are different. To show this, let $(N, v)$ be a game which is strictly convex. Let $\pi$ and $\sigma$ be two different permutations of $N$ and let $k$ be the 
smallest index for which $\pi_{k} \neq \sigma_{k}$. Furthermore, let $l>k$ be such that $\sigma_{l}=$ $\pi_{k}$. Define $S=\left\{\pi_{1}, \ldots, \pi_{k-1}\right\}$ and $T=\left\{\sigma_{1}, \ldots, \sigma_{l-1}\right\}$. Then $S \subset T$. Let $m^{\pi}$ denote the marginal allocation vector associated with the permutation $\pi$ and let $m^{\sigma}$ denote the one which is associated with $\sigma$. Then

$$
m_{\pi_{k}}^{\pi}=v\left(S \cup\left\{\pi_{k}\right\}\right)-v(S)<v\left(T \cup\left\{\pi_{k}\right\}\right)-v(T)=m_{\pi_{k}}^{\sigma} .
$$

It follows that $m^{\pi} \neq m^{\sigma}$. Hence, all marginal allocation vectors are different and consequently the core has precisely $n$ ! extreme elements (see also Shapley[49]). This shows that the bound in corollary 6.8 is sharp. To prove that the bound in theorem 6.17 is also sharp we need the following lemma.

Lemma 6.18 Let $(N, v)$ be a conver game, let $A \subseteq N$ and let $S \subseteq T \subseteq$ $N \backslash A$. Furthermore, let $\left(S_{i}\right)_{i=1}^{k}$ and $\left(T_{i}\right)_{i=1}^{k}$ be partitions of respectively $S$ and $T$, such that $S_{i} \subseteq T_{i}$ for all $i \in\{1, \ldots, k\}$. Then

$$
v(S \cup A)-\sum_{i=1}^{k} v\left(S_{i}\right) \leq v(T \cup A)-\sum_{i=1}^{k} v\left(T_{i}\right) .
$$

Moreover, when $v$ is strictly convex and $S \subset T$ then the inequality is strict.

Proof: When the partitions of $S$ and $T$ contain only one element, i.e. when $k=1$, the lemma follows directly from (6.1). Now, suppose the lemma is true when the partitions of $S$ and $T$ contain less than $k$ elements. Define $\bar{A}=S_{k} \cup A$. Observe that $\bar{A}$, the sets $S \backslash S_{k}$ and $T \backslash T_{k}$ and their partitions $\left(S_{i}\right)_{i=1}^{k-1}$ and $\left(T_{i}\right)_{i=1}^{k-1}$ satisfy the conditions of the lemma. According to the induction hypothesis we have

$$
v\left(S \backslash S_{k} \cup \bar{A}\right)-\sum_{i=1}^{k-1} v\left(S_{i}\right) \leq v\left(T \backslash T_{k} \cup \bar{A}\right)-\sum_{i=1}^{k-1} v\left(T_{i}\right) .
$$

From the definition of convexity it follows that

$$
v\left(T \backslash T_{k} \cup \bar{A}\right)+v\left(T_{k}\right) \leq v(T \cup A)+v\left(S_{k}\right) .
$$

Combining (6.4) and (6.5) we find

$$
v(S \cup A)-\sum_{i=1}^{k} v\left(S_{i}\right) \leq v(T \cup A)-\sum_{i=1}^{k} v\left(T_{i}\right) .
$$

When $v$ is strictly convex and $S \subset T$, we may assume without loss of generality that $S_{k} \subset T_{k}$. It follows that the inequality in (6.5) is strict, and hence also the inequality in (6.6) is strict. 
Theorem 6.19 Let $(N, v)$ be a strictly convex game and let $G=(N, E)$ be a cycle complete graph. Furthermore, let $B$ denote the collection of $G$ connected coalitions. Then Core $\left(B, v_{E}\right)$ has exactly $p_{G}$ extreme elements, where $v_{E}$ denotes the graph restricted game as defined in subsection 4.S.2.

Proof: Since coalitions outside $B$ are not essential in $v_{E}$, it follows that

$$
\operatorname{Core}\left(v_{E}\right)=\operatorname{Core}\left(B, v_{E}\right) \text {. }
$$

According to theorem 4.8 the graph restricted game $v_{E}$ is convex, so it follows that the extreme elements of $\operatorname{Core}\left(B, v_{E}\right)$ are precisely the marginal allocation vectors of $v_{E}$. We shall prove that $v_{E}$ has exactly $p_{G}$ different marginal allocation vectors. Let $\pi$ be a permutation of $N$ and consider the system of equations

$$
x\left(V_{\pi, k}\right)=v\left(V_{\pi, k}\right) \text { for all } k \in\{1, \ldots, n\},
$$

where the sets $V_{\pi, k}$ are defined as usual. According to lemma 6.10 the $G$ components of $U_{\pi, k}$ are members of $\left\{V_{\pi, 1}, \ldots, V_{\pi, k}\right\}$. Let $I$ denote the set of indices $i$ for which $V_{\pi, i}$ is a $G$-component of $U_{x, k}$. Any $x$ satisfying (6.7) also satisfies

$$
x\left(U_{i r, k}\right)=\sum_{i \in I} x\left(V_{x, i}\right)=\sum_{i \in I} v\left(V_{r, i}\right)=v_{E}\left(U_{\pi, k}\right)
$$

for all $k \in\{1, \ldots, \boldsymbol{n}\}$. It follows that system (6.7) has a unique solution, and this solution is the marginal vector of $v_{E}$ corresponding to the permutation $\pi$. Let us denote this marginal vector by $m^{\pi}$.

Let the permutations $\pi$ and $\sigma$ be such that the collections $\left\{V_{\pi, 1}, \ldots, V_{\pi, n}\right\}$ and $\left\{V_{\sigma, 1}, \ldots, V_{\sigma, n}\right\}$ are different. We shall prove that the vectors $m^{*}$ and $m^{\sigma}$ are different. Let $k$ be the smallest index such that $V_{\pi, k} \notin\left\{V_{\sigma, 1}, \ldots, V_{\sigma, n}\right\}$. Let $l$ be such that $\pi_{k}=\sigma_{l}$. According to lemma 6.10 all $G$-components of $V_{\pi, k}$ are members of $\left\{V_{\pi, 1}, \ldots, V_{\pi, k-1}\right\}$, and since $k$ is chosen such that $\left\{V_{\pi, 1}, \ldots, V_{\pi, k-1}\right\} \subset\left\{V_{e, 1}, \ldots, V_{\sigma, n}\right\}$, it follows that all $G$-components of $V_{\pi, k} \backslash\left\{\pi_{k}\right\}$ are members of $\left\{V_{\sigma, 1}, \ldots, V_{a, n}\right\}$. Now, let $V$ be a $G$-component of $V_{\pi, k} \backslash\left\{\pi_{k}\right\}$. Since $\sigma_{l}=\pi_{k}$ is adjacent to $V$, it follows that $V_{\sigma, l} \cup V$ is $G$ connected. Then, according to lemma 6.9, we must have either $V \subseteq V_{\sigma, l}$ or $V_{o, l} \subseteq V$. Since $\pi_{k} \in V_{o, l}$ and $\pi_{k} \notin V$, it follows that $V \subseteq V_{o, l}$. This is true for all components of $V_{\pi, k} \backslash\left\{\pi_{k}\right\}$, so we conclude that $V_{\pi, k} \backslash\left\{\pi_{k}\right\} \subseteq V_{o, l}$, and even $V_{\pi, k} \subseteq V_{\sigma, l}$, since $\pi_{k} \in V_{\sigma, l} . V_{\pi, k}$ was chosen such that $V_{x, k} \notin\left\{V_{\sigma, 1}, \ldots, V_{\sigma, n}\right\}$, so $V_{\pi, k} \neq V_{o, l}$ and it follows that $V_{\pi, k} \subset V_{o, l}$.

Let $\left(V_{i}\right)_{i=1}^{p}$ denote the partition of $V_{\pi, k} \backslash\left\{\pi_{k}\right\}$ into $G$-components, and let $\left(W_{i}\right)_{i=1}^{q}$ denote the partition of $V_{\sigma, l} \backslash\left\{\sigma_{l}\right\}$ into its $G$-components. Since $V_{i}$ is 
connected and $V_{i} \subseteq V_{\sigma, l}$, it follows that there must be a unique $G$-component of $V_{\sigma, l} \backslash\left\{\sigma_{l}\right\}$ which contains $V_{i}$ as a subset. Assume without loss of generality that $V_{i} \subseteq W_{i}$ for $i=1, \ldots, p$. Clearly, $p \leq q$. If $p<q$, we define $V_{i}=\emptyset$ for $i=p+1, \ldots, q$. This way we achieve that $\left(V_{i}\right)_{i=1}^{q}$ and $\left(W_{i}\right)_{i=1}^{q}$ are partitions of $V_{\pi, k} \backslash\left\{\pi_{k}\right\}$ and $V_{\sigma, l} \backslash\left\{\sigma_{l}\right\}$, satisfying $V_{i} \subseteq W_{i}$ for all $i \in\{1, \ldots, q\}$. Applying lemma 6.18 we find

$$
m_{\pi_{k}}^{\pi}=v\left(V_{\pi, k}\right)-\sum_{i=1}^{q} v\left(V_{i}\right)<v\left(V_{\sigma, l}\right)-\sum_{i=1}^{q} v\left(W_{i}\right)=m_{\sigma_{l}}^{\sigma}=m_{\pi_{k}}^{\sigma} .
$$

It follows that $m^{\pi} \neq m^{\sigma}$.

Since there are $p_{G}$ different collections $\left\{V_{\pi, 1}, \ldots, V_{\pi, n}\right\}$, it follows that there are exactly $p_{G}$ different marginal allocations.

\subsection{Computation of the permutation number}

In this section we provide some recursive rules for computing the permutation number of a graph.

Let $G$ be an undirected graph with vertex set $N$, and let $G_{1}, \ldots, G_{k}$ be the components of this graph. Then

$$
p_{G}=\Pi_{i=1}^{k} p_{G_{i}}
$$

Of course, applying this rule makes only sense when the graph $G$ is not connected.

Let $G$ be an undirected graph with vertex set $N$, and assume that $G$ is connected. Furthermore, let $\pi$ and $\sigma$ be permutations of $N$, such that $\pi_{n} \neq \sigma_{n}$. Then the collections $\left\{v_{\pi, 1}, \ldots, v_{\pi, n-1}\right\}$ and $\left\{v_{\sigma, 1}, \ldots, v_{\sigma, n-1}\right\}$ are different, because at least one of the vectors $v_{\sigma, i}$ has a 1 at place $\pi_{n}$, while the vectors $v_{\pi, i}$ have 0 at place $\pi_{n}$ for all $i \in\{1, \ldots, n-1\}$. Since $G$ is connected, we have $v_{\pi, n}=v_{\sigma, n}=1$, where 1 denotes the all-one vector. We conclude that $\left\{v_{\pi, 1}, \ldots, v_{\pi_{n}, n}\right\}$ and $\left\{v_{\sigma, 1}, \ldots, v_{\sigma, n}\right\}$ are different and hence the simplices $S_{G, \pi}$ and $S_{G, \sigma}$ are different.

Let $G^{i}$ denote the graph which results from $G$ by deleting the vertex $i$ from it. The number of different simplices in $S_{G}$, which result from permutations $\pi$ with $\pi_{n}=i$ is exactly the permutation number of the graph $G^{i}$. Consequently,

$$
p_{G}=\sum_{i \in N}\left|\left\{S_{G, r} \mid \pi_{n}=i\right\}\right|=\sum_{i \in N} p_{G^{i}}
$$


if $G$ is a connected graph.

Applying (6.8) and (6.9) and using that $p_{G}=1$ when $G$ has only one vertex, it is possible to compute the permutation number of any graph relatively easy, although an algorithm based on these rules will not be polynomial in the number of vertices of the graph.

For some types of graphs it is possible to give closed formulas for their permutation number. We give two examples.

Example: A complete graph. In this case we have $S_{G, \pi} \neq S_{G, \sigma}$ if $\pi \neq \sigma$. Therefore, the permutation number of a complete graph with $n$ vertices is $n$ !.

Example: A path. Let $s_{n}$ denote the permutation number of a path with $n$ vertices. Combining rules (6.8) and (6.9), we find that $s_{n}=$ $\sum_{i=0}^{n-1} s_{i} s_{n-i-1}$ if we adopt the convention $s_{0}=1$. This expression defines the well-known Catalan numbers. In [7] a closed formula is given for these numbers:

$$
s_{n}=\frac{1}{n+1}\left(\begin{array}{c}
2 n \\
n
\end{array}\right) .
$$

Since a path is a cycle complete graph, we conclude that the Catalan numbers form an upper bound for the number of extreme elements of $U(B, v)$ and Core $(B, v)$, when all elements of $B$ are path-connected.

Recall that the essential coalitions of the dual of a routing game are all path-connected. Hence, the number of extreme elements of the anti-core of a routing game with $n$ players is bounded by the $n$-th Catalan number. 


\section{Appendix A}

\section{Graph theoretic notions}

In this appendix we give the definitions of some basic graph theoretic notions. Other notions are defined when they first appear in the text.

Let $V$ be a non-empty finite set and let $E$ be a set of pairs of distinct elements of $V$. The ordered pair $G=(V, E)$ is called a graph. The graph is called directed if $E$ consists of ordered pairs. $G$ is called undirected if $E$ consists of unordered pairs. The elements of $V$ are called vertices or nodes, and the elements of $E$ are called arcs in case of a directed graph and they are called edges in case of an undirected graph. Assume from now on that $G$ is an undirected graph.

The vertices $v$ and $w$ are called the endpoints of the edge $\{v, w\}$.

Two distinct vertices $v, w \in V$ are called adjacent if $\{v, w\} \in E$.

The degree of a vertex $v \in V$ is the number of vertices which are adjacent to $v$.

A sequence of vertices $\left(v_{0}, v_{1}, \ldots, v_{k}\right)$ is called a path if $v_{i}$ and $v_{i+1}$ are adjacent for $i=0,1, \ldots, k-1 . v_{0}$ is called the initial vertex of the path and $v_{k}$ is called its final vertex.

A path $\left(v_{0}, \ldots, v_{k}\right)$ is called elementary if $v_{i} \neq v_{j}$ when $i \neq j$.

A path $\left(v_{0}, \ldots, v_{k}\right)$ is called a cycle if $k \geq 3, v_{0}=v_{k}$ and if no two other 
vertices on the path coincide.

Two paths with the same initial vertex $v$ and final vertex $w$ are called vertexdisjoint if $v$ and $w$ are the only vertices that these paths have in common.

The graph $G$ is called connected if for each pair of vertices $v, w \in V$ there exists a path with $v$ as initial vertex and $w$ as final vertex.
A forest is a graph without cycles.

A tree is a connected graph without cycles.

A complete graph is a graph for which any two vertices are adjacent.

A graph is called bipartite if all cycles contain an even number of distinct vertices.

The subgraph on $U \subseteq V$ of $G$ is the graph with vertex set $U$ and edge set $E_{U}=\{\{v, w\} \in E \mid v, w \in U\}$.

A component of $G$ is a maximal connected subgraph of $G$, that is, it is a connected subgraph, and there is no connected subgraph whose vertex set is strictly larger. Sometimes we shall say that a subset $U \subseteq V$ is a component of the graph $G$, when in fact the subgraph on $U$ is a component.

The complement of $G$ is the graph with vertex set $V$ and edge set $(V \times V) \backslash E$, where $V \times V$ denote the Cartesian product of $V$ and $V$. 


\section{Bibliography}

[1] H. Aarts and T.S.H. Driessen. Marginal allocations in the core of minimal chain games. Memorandum 1103, University of Twente, The Netherlands, 1992.

[2] H. Aarts and T.S.H. Driessen. On the core-invariance of minimum cost spanning tree games. Memorandum 1003, University of Twente, The Netherlands, 1992.

[3] R.J. Aumann and M. Maschler. The bargaining set for cooperative games. In M. Dresher, L.S. Shapley, and A.W. Tucker, editors, Advances in Game Theory, pages 443-476, Princeton University Press, Princeton, N.J., 1964.

[4] C. Berge. Graphs. North-Holland, 1985.

[5] C.G. Bird. On cost allocation for a spanning tree: a game theoretic approach. Networks, 6:335-350, 1976.

[6] G. Birkhoff and S. MacLane. A survey of modern algebra. Macmillan, New York, 1963.

[7] E. Catalan. Note sur une équation aux différences finies. Journal de Mathématiques Pures et Appliqués, 3:508-516, 1838.

[8] V. Chvatal. Linear Programming. Freeman, New York, 1983.

[9] A. Claus and D.J. Kleitman. Cost allocation for a spanning tree. Networks, 3:289-304, 1973.

[10] Y. Crama and F.C.R. Spieksma. Approximation algorithms for three dimensional assignment problems with triangle inequalities. European Journal of Operations Research, 60:273-279, 1992. 
[11] I.J. Curiel, G. Pederzoli, and S.H. Tijs. Sequencing games. European Journal of Operational Research, 40:344-351, 1989.

[12] I.J. Curiel. Assignment games and permutation games. Methods of Operations Research, 54:323 $\cdot 334,1986$.

[13] M. Davis and M. Maschler. The kernel of a cooperative game. Naval Research Logistics Quarterly, 12:223-259, 1965.

[14] J.J.M. Derks and J. Kuipers. On the Core and Nucleolus of Routing Games. Report M 92-07, RL Maastricht, the Netherlands, 1992 (to appear in International Journal of Game Theory).

[15] J.J.M. Derks and H. Reijnierse. On the Core of a Collection of Coalitions. Report M 93-12, RL Maastricht, the Netherlands, 1993.

[16] U. Faigle. Cores of games with restricted cooperation. Zeitschrift für Operations Research, 33:405-422, 1989.

[17] P.C. Fishburn and H.O. Pollack. Fixed route cost allocation. American Mathematical Monthly, 90:366-378, 1983.

[18] J. Fonlupt and D. Naddef. The traveling salesman problem in graphs with some excluded minors. Mathematical Programming, 53:147-172, 1992.

[19] D. Gale. Neighborly and cyclic polytopes. In V. Klee, editor, Convexity, Proceedings of Symposia in Pure Mathematics 7, pages 225-232, American Mathematical Society, 1963.

[20] M.R. Garey and D.S. Johnson. Computers and Intractability: A Guide to the Theory of NP-completeness. Freeman, San Francisco, 1979.

[21] M.C. Golumbic. Algorithmic Graph Theory and Perfect Graphs. Academic Press, Inc, 1980.

[22] D. Granot and F. Granot. On the computational complexity of a cost allocation approach to a fixed cost spanning forest problem. Mathematics of Operations Research, 17:765-780, 1993.

[23] D. Granot and G. Huberman. Minimum cost spanning tree games. Mathematical Programming, 21:1-18, 1981. 
[24] D. Granot and G. Huberman. On the core and nucleolus of minimum cost spanning tree games. Mathematical Programming, 29:323347,1984 .

[25] D. Hausmann, B. Korte, and T.A. Jenkyns. Worst case analysis of greedy type algorithms for independence systems. Mathematical Programming, 12:120-131, 1980.

[26] A.J. Hoffman, A. Kolen, and M. Sakarovitch. Totally balanced and greedy matrices. SIAM Journal on Algebraic and Discrete Methods, 6:721-730, 1985.

[27] M. Kaneko and M.H. Wooders, Cores of partitioning games. Mathematical Social Sciences, 3:313-327, 1982.

[28] J. Kuipers. A note on the 5-person traveling salesman game. Zeitschrift für Operations Research, 21:339-351, 1991.

[29] J. Kuipers. On the core of information graph games. International Journal of Game Theory, 21:339-351, 1993.

[30] J. Kuipers. On the LP-relaxation of multi-dimensional assignment problems with applications to assignment games. Report M 90-10, RL Maastricht, the Netherlands, 1990.

[31] J. Kuipers and J. Derks. On the number of extreme elements of a polyhedron. Report M 83-04, RL Maastricht, the Netherlands, 1993.

[32] P.S. Mara. Triangulations for the cube. Journal of Combinatorial Theory, Ser. A, 20:170-177, 1976.

[33] M. Maschler, B. Peleg, and L.S. Shapley. Geometric properties of the kernel, nucleolus, and related solution concepts. Methods of Operations Research, 4:303-338, 1979.

[34] M. Maschler, J.A.M. Potters, and S.H. Tijs. The general nucleolus and the reduced game property. International Journal of Game Theory, 21:83-106, 1992.

[35] P. McMullen. The maximum number of faces of a convex polytope. Mathematika, 17:179-184, 1970. 
[36] N. Megiddo. Computational complexity of the game theory approach to cost allocation for a tree. Mathematics of Operations Research, 3:189$195,1978$.

[37] N. Megiddo. Cost allocation for steiner trees. Networks, 8:1-6, 1978.

[38] R.B. Myerson. Graphs and cooperation in games. Mathematics of Operations Research, 2:224-229, 1977.

[39] (:.L. Nemhauser and I.A. Wolsey. Integer and Combinatorial Optimization. John Wiley and Sons, New York, 1988.

[40] A. van den Nouweland. Games and graphs in economic situations. Ph.D. Thesis, Tilburg, 1993.

[41] A. van den Nouweland and P. Borm. On the convexity of communication games. International Journal of Game Theory, 19:421-430, 1991.

[42] A. van den Nouweland, M. Maschler, and S.H. Tijs. Monotonic games are spanning network games. Discussion paper 9223, CentER for Economic Research, Tilburg University, The Netherlands, 1992.

[43] G. Owen. On the core of linear production games. Mathematical Programming, 9:358-370, 1975.

[44] G. Owen. Values of graph-restricted games. SIAM Journal of Algebraic and Discrete Models, 7:210-220, 1986.

[45] C.H. Papadimitriou and K. Steiglitz. Combinatorial Optimization: Algorithms and Complexity. Prentice-Hall, 1982.

[46] J.A.M. Potters, I.J. Curiel, and S.H. Tijs. Traveling salesman games. Mathematical Programming, 53:199-211, 1992.

[47] J.A.M. Potters, I.J. Curiel, and S.H. Tijs. Traveling salesman games. Report 8712, Catholic University Nijmegen, the Netherlands, 1987.

[48] D. Schmeidler. The nucleolus of a characteristic function game. SIAM Journal of Applied Mathematics, 17:1163-1170, 1969.

[49] L.S. Shapley. Cores of convex games. International Journal of Game Theory, 1:11-26, 1971. 
[50] L.S. Shapley. A value for n-person games. Annals of Mathematics Study, No. 28, Contributions to the Theory of Games, Vol. II, edited by H.W. Kuhn and A.W. Tucker, Princeton University Press, Princeton, New Jersey, 307-318, 1953.

[51] L.S. Shapley and M. Shubik. The assignment game i: the core. International Journal of Game Theory, 1:111-130, 1971.

[52] L.S. Shapley and M. Shubik. Quasi-cores in a monetary economy with non-convex preferences. Econometrica, 34:805-827, 1966.

[53] W.W. Sharkey. Network models in economics. Working paper, Bellcore, 1993.

[54] D. Skorin-Kapov. On the core of the minimum cost Steiner tree game in networks. Working paper, State University of New York at Stony Brook, 1993.

[55] D. Skorin-Kapov and H.F. Beltran. A unifying efficient characterization. of some cost allocation solutions associated with capacitated network design problems. Working paper, State University of Newv York at Stony Brook, 1993.

[56] A. Tamir. On the core of cost allocation games defined on location problems. Technical report, Department of Statistics, Tel Aviv University, 1980.

[57] A. Tamir. On the core of a traveling salesman cost allocation game. Operation Research Letters, 8:31-34, 1989.

[58] R. Tarjan. Depth-first search and linear graph algorithms. SIAM Journal of Computation, 1:146-160, 1972.

[59] S.H. Tijs and T.S.H. Driessen. Extensions of solution concepts by means of multiplicative $\epsilon$-tax games. Mathematical Social Sciences, 12:9-20, 1986.

[60] Stef H. Tijs. Bounds for the core and the $\tau$-value. In: Game Theory and Mathematical Economics (Eds. O. Moeschlin and D. Pallaschke) North-Holland Publishing Company, Amsterdam, 123-132, 1981. 



\section{Subject index}

2-connected graph 74

affinely independent 118

allocation 2

allocation problem 1

anti-imputation set 4

arc 135

bipartite graph 136

block 74

capacitated network design game 93

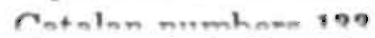

characteristic function 2

chord 17

chordal graph 17

clique 12

maximal 12

communication situation $\mathbf{7 7}$

complement graph 136

complete graph 136

component 136

component increasing graph 126

concave game 73

connected graph 136

contracted set 100

convex game 129

core 3

anti- 4

B-restricted 7

$\epsilon-112$

k- 112

least tax 112

core problem 9 
cycle 135

cycle complete graph $\mathbf{7 4}$

dependent player

$$
\text { B- } 15
$$

directed graph 135

dual game 5

edge 135

minimal 95

edge contraction 50

edge deletion 50

essential coalition 8

anti- 26

fixed cost spanning forest game 93

fixed-route traveling salesman game 40

forest 136

fractional covering problem 33

fractional partitioning problem 9

graph induced polytope 121

graph restricted game 77,131

Helly property 13

imputation set 4

incidence vector 24

inclusion property 34

individually rational 3

information graph 73

information graph game 72

informed set 73

interior 116

interior point 116

intersection graph 12

location game 38

location problem 37

marginal allocation vector 61 
minimum cost forest game 93

minimum cost spanning tree game 59

minimum excess coalition structure theorem 62

minimum length tour $\mathbf{4 7}$

minor graph 50

monotonic minimum cost spanning tree game 61

multi-assignment game 113

multi-dimensional assignment game 111

multi-dimensional assignment problem 105

nearest neighbour tour 48

network 64

minimal 95

node 135

$\mathrm{N}$-subadditive $\mathbf{4 2}$

nucleolus 4

anti- 4

B- 21

partition

B- 8

maximal $B-8$

partitioning game 8

path 135

elementary 135

perfect collection 9

perfect graph 12

perfect matrix 9

permutation number 128

player set 2

p-normalized game $\mathbf{5 2}$

positive anti-core problem 33

prenucleolus 4

anti- 4

B- 21

routing game 40

row inclusion matrix 34

sequencing game 20,44 
Shapley value 3

simple game 20

simplex 118

simplicial subdivision 118

solution concept 3

spanning network game 93

stable 11

B- 11

Steiner tree game 93, 99

strategically equivalent $\mathbf{4 7}$

strictly convex game 129

total dependency property 15

total inclusion property 34

totally balanced matrix 34

transferable utility game 2

traveling salesman game 39

tree 136

tree vector 60

triangulated graph $\mathbf{1 7}$

triangulation 119

triangle inequalities 46

$\tau$-value 3

undirected graph 135

unit hypercube 116

upper set 115

vertex 135

adjacent 135

final 135

initial 135

separating 81

vertex-disjoint paths 136

veto player 20

weak demand operation 62

weak triangle inequalities $\mathbf{4 7}$

worth 2 


\section{Author index}

Aarts, H. 70, 94, 95

Aumann, R.J. 3

Beltran, H.F. 93

Berge, C. 78

Bird, C.G. 59

Birkhoff, G. 119

Borm, P. 74, 77

Catalan, E. 133

Claus, A. 59

Crama, Y. 106

Curiel, I.J. 20, 39, 44, 47, 113

Davis, M. 3

Derks, J.J.M. 7, 39, 115

Driessen, T.S.H. 70, 94, 95, 112

Faigle, U. 7

Fishburn, P.C. 39

Fonlupt, J. 40, 50

Gale, D. 116

Garey, M.R. 106

Golumbic, M.C. 9, 12, 17

Granot, D. 21, 59, 60, 61, 62, 93

Granot, F. 93

Hausmann, D. 108

Hoffman, A.J. 33, 38

Huberman, G. 21, 59, 60, 61, 62

Jenkyns, T.A. 108

Johnson, D.S. 106
Kaneko, M. 8, 9, 11, 15

Kleitman, D.J. 59

Kolen, A. 33i

Korte, B. 108

Kuipers, J. 39, 59, 105, 115

Maclane, S. 119

Mara, P.S. 119

Maschler, M. 3, 21, 22, 93

McMullen, P. 116

Megiddo, N. 72, 93

Myerson, R.B. 6, 7

Naddef, D. 40, 50

Nemhauser, G.L. 10, 12, 15, 34

Nouweland, A. van den 74, 77, 93

Owen, G. 6, 7, 47

Papadimitriou, C.H. 49

Pederzoli, G. 20, 14

Peleg, B. 3, 22

Pollack, H.O. 39

Potters, J.A.M. 21, 39, 47

Reijnierse, H. 7

Sakarovitch, M. 33

Schmeidler, D. 3, 4, 24

Shapley, L.S. 3, 22, 84, 111, 112, 130

Sharkey, W.W. 5

Shubik, M. 111, 112

Skorin-Kapov, D. 93

Spieksma, F.C.R. 106 
Steiglitz, K. 49

Tamir, A. 38, 40

Tarjan, R. 80

Tijs, T.S.H. 3, 20, 21, 39, 44, 47, 93, 112, 113

Wolsey, L.A. 10, 12, 15, 34

Wooders, M.H. 8, 9, 11, 15 


\section{Samenvatting}

In dit proefschrift beschouwen we situaties waar een groep van individuen een samenwerkingsverband kan aangaan. Door het sluiten van zo'n onderlinge verbintenis zal de groep in het algemeen efficienter kunnen functioneren, dan wanneer de individuen afzonderlijk werken. Dit maakt samenwerking aantrekkelijk. De individuen worden spelers genoemd. We nemen aan dat niet alleen de groep van alle spelers kan samenwerken, maar dat ook elke deelgroep van spelers een onderlinge verbintenis kan aangaan. Een samenwerkingsverband van een deelgroep wordt een coalitie genoemd. De karakteristieke functie geeft weer welke kosten of winsten aan iedere mogelijke coalitie gekoppeld zijn. Spelersverzameling en karakteristieke functie tesamen noemen we een cooperatief spel.

De cooperatieve speltheorie is de tak van wiskunde die bovenstaande situaties beschrijft en bestudeert. Twee probleemstellingen zijn van belang: welke coalities zullen zich vormen en hoe moeten de gemaakte winsten of kosten van een coalitie verdeeld worden over de spelers in zo'n coalitie. Vaak is het niet onredelijk om te veronderstellen dat alle spelers in een spel zullen samenwerken in wat we de grote coalitie zullen noemen. Ook in dit proefschrift zullen we telkens deze veronderstelling maken. We concentreren ons op de vraag hoe de kosten of winsten van de grote coalitie verdeeld moeten worden over de spelers.

Een methode die voorschrijft hoe deze verdeling moet plaatsvinden wordt een oplossingsconcept genoemd. Tal van oplossingsconcepten zijn in de literatuur beschreven, zoals de core, de nucleolus en de Shapley waarde. Bij een spel met $n$ spelers kunnen zich $2^{n}-1$ coalities vormen. Voor de zojuist genoemde oplossingsconcepten kan de waarde van iedere coalitie van belang zijn in hun berekening, zodat deze berekening al snel een praktisch onmogelijke taak wordt.

Om deze reden zijn er oplossingsconcepten bedacht waarvoor de berekening slechts een deel van de karakteristieke functie vereist. Ook wordt onderzoek verricht naar klassen van spelen waarbij de karakteristieke functie een zodanige structuur heeft dat de gangbare oplossingsconcepten eenvoudig berekenbaar zijn. Beide onderzoekslijnen raken elkaar als voor een bepaalde klasse van spelen het vereenvoudigde oplossingsconcept met een gangbaar oplossingsconcept overeenkomt. 
Dit proefschrift kan men zien in het licht van dit type onderzoek. We behandelen verschillende klassen van cooperatieve spelen waarbij de karakteristieke functie van het spel bepaald wordt door een combinatorisch optimaliseringsprobleem. Een voorbeeld hiervan is de klasse van minimaal opspannende boom spelen, waarbij de waarde van een coalitie bepaald wordt door een minimaal opspannende boom probleem, een bekend probleem uit de combinatorische optimalisering. De karakteristieke functie van minimaal opspannende boom spelen blijkt een structuur te hebben die de analyse van het spel vereenvoudigt. We onderzoeken de eigenschappen van de core en de nucleolus voor een aantal klassen van spelen die samenhangen met een probleem uit de combinatorische optimalisering.

Hoofdstuk 1 van het proefschrift is inleidend. In hoofdstuk 2 beschouwen we spelen waarbij slechts een deel van de coalities zich werkelijk kan vormen. We geven condities op deze collectie van formeerbare coalities die het niet leeg zijn van de core garanderen en die berekening van een core element of de nucleolus aanmerkelijk vereenvoudigen.

In hoofdstuk 3 beschouwen we spelen die gerelateerd zijn aan het handelsreizigersprobleem. Bij een bepaalde variant van dit type spelen, de routeringsspelen, blijkt slechts een klein deel van de coalities relevant te zijn voor berekening van de core en de nucleolus. Hier kunnen de resultaten uit hoofdstuk 2 gebruikt worden voor een efficiente berekening van deze oplossingsconcepten.

Hoofstuk $\mathbf{4}$ behandelt verschillende varianten van spelen die verband houden met het minimaal opspannende boom probleem. Ook hier blijkt weer dat de resultaten uit hoofdstuk 2 toegepast kunnen worden om berekening van core elementen en de nucleolus te vereenvoudigen.

In hoofdstuk 5 beschouwen we spelen waarbij de coalitie waarden bepaald worden door een meer-dimensionaal toekenningsprobleem. Omdat de core yoor dit soort spelen leeg kan zijn, introduceren we een verwant oplossingsconcept, de $k$-core, en we analyseren enkele eigenschappen van de $k$-core met betreking tot meer-dimensionale toekenningsspelen.

Tenslotte, in hoofdstuk 6, stellen we onze interesse in het aantal extreme punten van de core. We tonen aan dat dit aantal ten hoogste $n$ ! is als $n$ het aantal spelers in het spel is. We geven ook bovengrenzen voor dit aantal als slechts een deel van de coalities formeerbaar is. Een van de nevenresultaten van dit onderzoek laat zien dat de core van een routeringsspel met $n$ spelers ten hoogste $\frac{1}{n+1}\left(\begin{array}{c}2 n \\ n\end{array}\right)$ extreme punten heeft. 


\section{Curriculum vitae}

Jeroen Kuipers werd geboren op 7 januari 1963 te West Souburg. Van 1975 tot 1981 volgde hij voorbereidend wetenschappelijk onderwijs aan de Stedelijke Scholengemeenschap te Middelburg. Na het behalen van zijn eindexamen begon hij in september 1981 met de studie Toegepaste Wiskunde aan de Technische Universiteit Eindhoven. In 1985 behaalde hij in deze studie het kandidaatsexamen (met lof). Zijn afstudeerstage verrichtte hij van augustus 1987 tot juni 1988 bij het Dr. Neher Laboratorium te Leidschendam en eind juni 1988 legde hij het doctoraal examen af.

Per 1 augustus 1988 trad hij in dienst bij de vakgroep wiskunde van de Rijksuniversiteit Limburg. Aanvankelijk verrichtte hij hier onderzoek naar het onderwerp van concurrerende doodsoorzaken. Van 1 januari 1990 tot en met 31 december 1993 was hij werkzaam als AIO bij dezelfde vakgroep op het gebied van de cooperatieve speltheorie. De resultaten van dit onderzoek zijn weergegeven in dit proefschrift.

Sinds 1 januari 1994 is de schrijver van dit proefschrift werkzaam als toegevoegd onderzoeker, nog steeds bij de vakgroep wiskunde van de Rijksuniversiteit Limburg. 
